\title{
Cool clusters correctly correlated
}

\author{
by
}

Sergey Aleksandrovich Varganov

A dissertation submitted to graduate faculty

in partial fulfillment of the requirements for the degree of

DOCTOR OF PHILOSOPHY

\author{
Major: Physical Chemistry \\ Program of Study Committee: \\ Mark S. Gordon, Major Professor \\ Gordon J. Miller \\ Xueyu Song \\ Monica H. Lamm \\ James W. Evans
}

Iowa State University

Ames, Iowa 
Graduate College

Iowa State University

This is to certify that the doctoral dissertation of

Sergey Aleksandrovich Varganov

has met the dissertation requirements of Iowa State University

Committee Member

Committee Member

Committee Member

Committee Member

Major Professor

For the Major Program 


\section{TABLE OF CONTENTS}

CHAPTER 1. GENERAL INTRODUCTION 1

I. General overview 1

II. Methods for solving the electronic Schrödinger equation 2

A. Born-Oppenheimer approximation 2

B. Hartree-Fock method 2

C. Configuration interaction 7

D. Møller-Plesset perturbation theory 9

E. Coupled Cluster method 11

F. Methods based on multireference wave functions 13

G. Density functional theory 15

III. Dissertation organization 17

$\begin{array}{ll}\text { References } & 18\end{array}$

CHAPTER 2. THE INTERACTION OF OXYGEN WITH SMALL GOLD CLUSTERS 20

Abstract 20

I. Introduction 21

II. Computational methods 22

III. Results and discussion 23

A. Results for $\mathrm{Au}_{2}$

B. Results for $\mathrm{Au}_{3}$

C. Results for $\mathrm{Au}_{3}^{-}$

D. Results for $\mathrm{Au}_{2} \mathrm{O}_{2}$ and $\mathrm{Au}_{2}\left(\mathrm{O}_{2}\right)_{2}$

E. Results for $\mathrm{Au}_{2} \mathrm{O}_{2}^{-}$and $\mathrm{Au}_{2}\left(\mathrm{O}_{2}\right)_{2}^{-}$

F. Oxygen binding to $\mathrm{Au}_{3}$

G. Oxygen binding to $\mathrm{Au}_{3}{ }^{-}$

IV. Summary 30

Acknowledgments 31

References $\quad 32$

CHAPTER 3. A STUDY OF THE REACTION OF MOLECULAR HYDROGEN WITH SMALL GOLD CLUSTERS

Abstract 44

I. Introduction 44

II. Computational methods 46

III. Results and discussion $\quad 48$

IV. Summary 52

Acknowledgments $\quad 54$

References $\quad 54$

CHAPTER 4. A STUDY OF THE ISOMERS OF $C_{36}$ FULLERENE USING SINGLE AND MULTIREFERENCE MP2 PERTURBATION THEORY

Abstarct 
I. Introduction 65

II. Computational details 67

III. . Results and discussion $\quad 68$

IV. Summary $\quad 71$

Acknowledgments $\quad 71$

$\begin{array}{ll}\text { References } & 71\end{array}$

CHAPTER 5. EFFECTS OF STRONG ELECTRON CORRELATIONS IN $\mathrm{Ti}_{8} \mathrm{C}_{12}$ $\begin{array}{ll}\text { MET-CAR } & 79\end{array}$

$\begin{array}{ll}\text { Abstract } & 79\end{array}$

$\begin{array}{ll}\text { I. Introduction } & 79\end{array}$

II. Computational methods $\quad 81$

III. Results and discussion $\quad 82$

A. Model for $\mathrm{Ti}_{8} \mathrm{C}_{12}$ electron structure 82

B. Multireference calculations $\quad 85$

C. Single reference calculations 87

D. Calculation of ionization potential 89

IV. Conclusions 90

Acknowledgments $\quad 91$

$\begin{array}{ll}\text { References } & 91\end{array}$

CHAPTER 6. DENSITY CUMULANT FUNCTIONAL THEORY 106

Abstract 106

I. Introduction 106

II. Energy as a functional of the 1-RDM and cumulant of 2-RDM 109

III. Parametrization of diagonal part of cumulant 111

IV. Optimization of energy functional 114

V. Preliminary results 115

VI. Summary 116

Acknowledgments 117

$\begin{array}{ll}\text { References } & 117\end{array}$

$\begin{array}{ll}\text { CHAPTER 7. GENERAL CONCLUSIONS } & 120\end{array}$

$\begin{array}{ll}\text { ACKNOWLEDGMENTS } & 123\end{array}$ 


\section{CHAPTER 1. GENERAL INTRODUCTION}

\section{General overview}

Atomic clusters are unique objects, which occupy an intermediate position between atoms and condensed matter systems. For a long time it was thought that physical and chemical properties of atomic clusters monotonically change with increasing size of the cluster from a single atom to a condensed matter system. However, recently it has become clear that many properties of atomic clusters can change drastically with the size of the clusters. Because physical and chemical properties of clusters can be adjusted simply by changing the cluster's size, different applications of atomic clusters were proposed. One example is the catalytic activity of clusters of specific sizes in different chemical reactions. Another example is a potential application of atomic clusters in microelectronics, where their band gaps can be adjusted by simply changing cluster sizes.

In recent years significant advances in experimental techniques allow one to synthesize and study atomic clusters of specified sizes. However, the interpretation of the results is often difficult. The theoretical methods are frequently used to help in interpretation of complex experimental data. Most of the theoretical approaches have been based on empirical or semiempirical methods. These methods allow one to study large and small clusters using the same approximations. However, since empirical and semiempirical methods rely on simple models with many parameters, it is often difficult to estimate the quantitative and even qualitative accuracy of the results. On the other hand, because of significant advances in quantum chemical methods and computer capabilities, it is now possible to do high quality ab-initio calculations not only on systems of few atoms but on clusters of practical interest as well. In addition to accurate results for specific clusters, such methods can be used for benchmarking of different empirical and semiempirical approaches.

The atomic clusters studied in this work contain from a few atoms to tens of atoms. Therefore, they are quantum objects. Some qualitative information about the geometries of such clusters can be obtained with classical empirical methods, for example geometry optimization using an empirical Lennard-Jones potential ${ }^{1}$. However, to predict their accurate 
geometries and other physical and chemical properties it is necessary to solve a Schrödinger equation. If one is not interested in dynamics of clusters it is enough to solve the stationary (time-independent) Schrödinger equation.

$$
H \Phi=E \Phi
$$

This equation represents a multidimensional eigenvalue problem. The solution of the Schrödinger equation is a set of eigenvectors (wave functions) and their eigenvalues (energies). The lowest energy solution (wave function) corresponds to the ground state of the cluster. The other solutions correspond to excited states. The wave function gives all information about the quantum state of the cluster and can be used to calculate different physical and chemical properties, such as photoelectron, X-ray, NMR, EPR spectra, dipole moment, polarizability etc.

The dimensionality of the Schrödinger equation is determined by the number of particles (nuclei and electrons) in the cluster. The analytic solution is only known for a two particle problem. In order to solve the equation for clusters of interest it is necessary to make a number of approximations and use numerical methods.

\section{Methods for solving the electronic Schrödinger equation}

\section{A. Born-Oppenheimer approximation}

Since masses of nuclei are significantly larger than the mass of an electron, the Schrödinger equation can be split into two coupled electronic and nuclear equations. The electronic Schrödinger equation describes the electrons moving in the potential of fixed nuclei. The nuclear equation describes the nuclei moving in the potential created by the electrons. Such a potential is called a potential energy surface and the approximation is called the Born-Oppenheimer approximation².

\section{B. Hartree-Fock method}

The electronic Schrödinger equation can be written as 


$$
\begin{aligned}
& \mathbf{H}_{e} \Phi_{e}=E_{e} \Phi_{e} \\
& \mathbf{H}_{e}=\sum_{j=1}^{N} \mathbf{h}_{i}+\sum_{i=1}^{N} \sum_{j>i}^{N} r_{i j}^{-1} \\
& \mathbf{h}_{i}=-\frac{1}{2} \nabla_{i}^{2}-\sum_{a} \frac{Z_{a}}{\left|\mathbf{R}_{a}-\mathbf{r}_{i}\right|} \\
& r_{i j}^{-1}=\frac{1}{\left|\mathbf{r}_{i}-\mathbf{r}_{j}\right|}
\end{aligned}
$$

Here the electronic Hamiltonian $\mathbf{H}_{e}$ consists of one-electron operators $\mathbf{h}_{i}$, and two-electron operators $r_{i j}^{-1}$. Each $\mathbf{h}_{j}$ is the sum of electron kinetic energy and electron-nuclei interaction operators. The two-electron operator $r_{i j}^{-1}$ describes the coulomb repulsion between electrons. Since electrons are fermions, the wave function of an electronic system must be antisymmetric with respect to exchange of the coordinates of any two electrons. This property of the wave function can be guaranteed by representing it as linear combination of Slater determinants. The columns of such determinants contain the one-electron wave functions $\psi_{i}$. These one-electron wave functions are called molecular orbitals, and can be represented as products of spatial orbitals and spin functions.

$$
\Psi=\frac{1}{\sqrt{N !}}\left|\begin{array}{cccc}
\psi_{1}(1) & \psi_{2}(1) & \ldots & \psi_{N}(1) \\
\psi_{1}(2) & \psi_{2}(2) & \ldots & \psi_{N}(2) \\
\ldots & \ldots & \ldots & \ldots \\
\psi_{1}(N) & \psi_{2}(N) & \ldots & \psi_{N}(N)
\end{array}\right|
$$

If the wave function of a system with an even number of electrons is represented as only one Slater determinant then it is called a Hartree-Fock type wave function.

Since $\Psi$ is normalized, the electronic energy can be calculated as $E_{e}=\left\langle\Psi\left|H_{e}\right| \Psi\right\rangle$. If the molecular spin orbitals $\psi_{i}$ are normalized then the total electron energy can be written as 


$$
\begin{aligned}
& E_{e}=\sum_{i=1}^{N} h_{i}+\frac{1}{2} \sum_{i=1}^{N} \sum_{j=1}^{N}\left(J_{i j}-K_{i j}\right) \\
& h_{i}=\left\langle\psi_{i}(1)\left|h_{1}\right| \psi_{i}(1)\right\rangle \\
& J_{i j}=\left\langle\psi_{i}(1) \psi_{j}(2)\left|r_{12}^{-1}\right| \psi_{i}(1) \psi_{j}(2)\right\rangle \\
& K_{i j}=\left\langle\psi_{i}(1) \psi_{j}(2)\left|r_{12}^{-1}\right| \psi_{i}(2) \psi_{j}(1)\right\rangle
\end{aligned}
$$

The matrix elements $h_{i}$ are called one-electron integrals. The $J_{i j}$ and $K_{i j}$ are two-electron coulomb and exchange integrals, respectively.

Now, it is necessary to find that set of molecular orbitals $\psi_{i}$, which minimizes the functional of the electronic energy. It is possible to show that such molecular orbitals have to satisfy the Hartree-Fock equations:

$$
\mathbf{F}_{i} \psi_{i}=\sum_{j}^{N} \varepsilon_{i j} \psi_{j}
$$

Here $\mathbf{F}_{i}$ is a Fock operator defined as a sum of the one-electron operator $\mathbf{h}_{i}$ and the twoelectron coulomb $\mathbf{J}_{j}$ and exchange $\mathbf{K}_{j}$ operators .

$$
\begin{aligned}
& \mathbf{F}_{i}=\mathbf{h}_{i}+\sum_{j}^{N}\left(\mathbf{J}_{j}-\mathbf{K}_{j}\right) \\
& \mathbf{J}_{j}\left|\psi_{i}(2)\right\rangle=\left\langle\psi_{j}(1)\left|r_{12}^{-1}\right| \psi_{j}(1)\right\rangle\left|\psi_{i}(2)\right\rangle \\
& \mathbf{K}_{j}\left|\psi_{i}(2)\right\rangle=\left\langle\psi_{j}(1)\left|r_{12}^{-1}\right| \psi_{i}(1)\right\rangle\left|\psi_{j}(2)\right\rangle
\end{aligned}
$$

Molecular orbitals satisfying equation (5) are not unique. However, it is possible to find a set of molecular orbitals that the makes the matrix $\varepsilon$ diagonal. Such orbitals are called canonical molecular orbitals. In the basis of canonical orbitals equation (5) becomes

$$
\mathbf{F}_{i} \psi_{i}=\varepsilon_{i} \psi_{i}
$$


The eigenvalues $\varepsilon_{i}$ of the Fock operator are interpreted as the energies of the canonical molecular spin orbitals $\psi_{i}$. In order to determine the optimal molecular orbitals, it is necessary to know the matrix representation of the Fock operator, which depends on the molecular orbitals. Therefore, the Hartree-Fock equation has to be solved iteratively.

For small systems, such as atoms and diatomic molecules, the Hartree-Fock equations can be solved by mapping the molecular orbitals onto a discrete grid. This aproach is often called the numerical Hartree-Fock method. For large systems it is more practical to expand molecular orbitals in the basis of some known functions. In general, this basis can consist of any type of functions that forms a complete basis in Hilbert space. However, it is advantagous to chose a basis set that guarantees fast convergence of the energy and wave function with respect to the number of basis functions. Another requirement for the basis set is the possibility of fast calculations of matrix elements of the one- and two-electron operators. Experience has demonstrated that the optimal balance between these two requirements can be achieved by using gaussian type basis functions. Such functions can be written in spherical (Eq. 8a) or Cartesian (Eq. 8b) coordinates.

$$
\begin{aligned}
& \chi_{\zeta, n, l, m}(r, \theta, \varphi)=N Y_{l, m}(\theta, \varphi) r^{(2 n-2-l)} e^{-\zeta^{2}} \\
& \chi_{\zeta, l_{x}, l_{y}, l_{z}}(x, y, z)=N x^{l_{x}} y^{l} z^{l} e^{-\zeta^{2}}
\end{aligned}
$$

Here $l_{x}, l_{y}$ and $l_{z}$ are orbital quantum numbers (for example, $l_{x}+l_{y}+l_{z}=1$ is defined as a p-type atomic orbital). Using these basis functions, the molecular orbitals $\psi_{i}$ can be represented as linear combinations of $M$ gaussian functions $\chi_{\alpha}$.

$$
\psi_{i}=\sum_{\alpha}^{M} c_{\alpha i} \chi_{\alpha}
$$

Inserting (9) into the Hartree-Fock equation (7) produces

$$
\mathbf{F}_{i} \sum_{\alpha}^{M} c_{\alpha i} \chi_{\alpha z}=\varepsilon_{i} \sum_{\alpha}^{M} c_{\alpha i} \chi_{\alpha}
$$


Multiplying Eq. (10) on the left by $\chi_{\beta}$ and integrating leads to the Roothaan-Hall equation ${ }^{3,4}$. In matrix form all $M$ equations can be written as

$$
\begin{aligned}
& \mathbf{F C}=\mathbf{S C E} \\
& F_{\alpha \beta}=\left\langle\chi_{\alpha}|\mathbf{F}| \chi_{\beta}\right\rangle \\
& S_{\alpha \beta}=\left\langle\chi_{\alpha} \mid \chi_{\beta}\right\rangle
\end{aligned}
$$

Here $\mathbf{F}$ is the Fock matrix, $\mathbf{C}$ is the matrix of coefficients, $\mathbf{S}$ is the overlap matrix and $\mathbf{E}$ is the diagonal matrix of molecular orbital energies $\varepsilon_{i}$. The elements of the Fock matrix in the basis of gaussian functions $\chi$ can be written as

$$
\begin{aligned}
\left\langle\chi_{\alpha}|\mathbf{F}| \chi_{\beta}\right\rangle & =\left\langle\chi_{\alpha}|\mathbf{h}| \chi_{\beta}\right\rangle+\sum_{j}^{o c c . M O}\left\langle\chi_{\alpha}\left|\mathbf{J}_{j}-\mathbf{K}_{j}\right| \chi_{\beta}\right\rangle \\
& =\left\langle\chi_{\alpha}|\mathbf{h}| \chi_{\beta}\right\rangle+\sum_{j}^{o c c . M O}\left(\left\langle\chi_{\alpha} \psi_{j}\left|r_{12}^{-1}\right| \chi_{\beta} \psi_{j}\right\rangle-\left\langle\chi_{\alpha} \psi_{j}\left|r_{12}^{-1}\right| \psi_{j} \chi_{\beta}\right\rangle\right) \\
& =\left\langle\chi_{\alpha}|\mathbf{h}| \chi_{\beta}\right\rangle+\sum_{j}^{o c c . M O A O} \sum_{\gamma}^{A O} \sum_{\delta}^{A O} c_{\gamma j} c_{\delta j}\left(\left\langle\chi_{\alpha} \chi_{\gamma}\left|r_{12}^{-1}\right| \chi_{\beta} \chi_{\delta}\right\rangle-\left\langle\chi_{\alpha} \chi_{\gamma}\left|r_{12}^{-1}\right| \chi_{\delta} \chi_{\beta}\right\rangle\right) \\
& =\left\langle\chi_{\alpha}|\mathbf{h}| \chi_{\beta}\right\rangle+\sum_{\gamma}^{A O} \sum_{\delta}^{A O} P_{\gamma \delta}\left(\left\langle\chi_{\alpha} \chi_{\gamma}\left|r_{12}^{-1}\right| \chi_{\beta} \chi_{\delta}\right\rangle-\left\langle\chi_{\alpha} \chi_{\gamma}\left|r_{12}^{-1}\right| \chi_{\delta} \chi_{\beta}\right\rangle\right) \\
P_{\gamma \delta}= & \sum_{j}^{o r c . M O} c_{\gamma j} c_{\delta j}
\end{aligned}
$$

Matrix $\mathbf{P}$ with elements $P_{\gamma \delta}$ is called the density matrix.

The electron energy can be written as 


$$
\begin{aligned}
E_{e} & =\sum_{i}^{N}\left\langle\psi_{i}\left|\mathbf{h}_{i}\right| \psi_{i}\right\rangle+\frac{1}{2} \sum_{i j}^{N}\left(\left\langle\psi_{i} \psi_{j}\left|r_{12}^{-1}\right| \psi_{i} \psi_{j}\right\rangle-\left\langle\psi_{i} \psi_{j}\left|r_{12}^{-1}\right| \psi_{j} \psi_{i}\right\rangle\right) \\
& =\sum_{i}^{N} \sum_{\alpha \beta}^{M} c_{\alpha i} c_{\beta i}\left\langle\chi_{\alpha}\left|\mathbf{h}_{i}\right| \chi_{\beta}\right\rangle+\frac{1}{2} \sum_{i j}^{N} \sum_{\alpha \beta \gamma \delta}^{M} c_{\alpha i} c_{y j} c_{\beta i} c_{\delta j}\left(\left\langle\chi_{\alpha \alpha} \chi_{\gamma}\left|r_{12}^{-1}\right| \chi_{\beta} \chi_{\delta}\right\rangle-\left\langle\chi_{\alpha} \chi_{\gamma}\left|r_{12}^{-1}\right| \chi_{\delta} \chi_{\beta}\right\rangle\right) \\
& =\sum_{\alpha \beta}^{M} P_{\alpha \beta} h_{\alpha \beta}+\frac{1}{2} \sum_{\alpha \beta \gamma \delta}^{M} P_{\alpha \beta} P_{\gamma \delta}\left(\left\langle\chi_{\alpha} \chi_{\gamma}\left|r_{12}^{-1}\right| \chi_{\beta} \chi_{\delta}\right\rangle-\left\langle\chi_{\alpha} \chi_{\gamma}\left|r_{12}^{-1}\right| \chi_{\delta} \chi_{\beta}\right\rangle\right)
\end{aligned}
$$

In calculations on closed shell singlet states (all electrons are paired) a so called restricted Hartree-Fock method is used. One cannot realistically use the Hartree-Fock method for calculations on singlet open-shell states (for example, calculations on diradicals) because in this case more than one Slater determinant make significant contributions to the total wave function. However, there are two popular adaptations of the Hartree-Fock method for calculations on high-spin open-shell systems, which contain one or more unpaired electrons with the same spin. In the restricted open-shell Hartree-Fock (ROHF) theory paired electrons occupy the same molecular orbital. In the unrestricted Hartree-Fock (UHF) method each electron occupies a separate molecular orbital. Computationally the UHF method is aproximately twice as expensive as ROHF, since there are twice more molecular orbitals in UHF. In addition, the UHF wave function is not an eigenfunction of the $\mathbf{S}^{2}$ operator. This means that a UHF wave function contains contributions from several spin states. For example the UHF wave function of a triplet state can be contaminated by contributions from wave functions of higher multiplicity (quintet, septet, etc.) states.

The Hartree-Fock approximation is an example of a mean-field theory. Each electron interacts with an average field generated by all other electrons. In reality the behavior of each electron explicitly depends on positions and momenta of all other electrons. In other words electrons are correlated. The difference between the exact electronic energy and the HartreeFock energy is referred to as the correlation energy.

\section{Configuration interaction}

The configuration interaction (CI) method is conceptually the simplest approach to calculate the correlation energy and improve the HF approximation. The exact wave function 
for a given atomic basis can be expanded in a sum of Slater determinants generated by all possible substitutions of occupied molecular orbitals with unoccupied ones in the HartreeFock determinant.

$$
\Phi=\Psi_{H F}+\sum_{i}^{o c c} \sum_{a}^{y i r} C_{i}^{a} \Psi_{i}^{a}+\sum_{j<j}^{o c c} \sum_{a<b}^{v i r} C_{i j}^{a b} \Psi_{i j}^{a b}+\ldots
$$

Here $\Psi_{H F}$ is the Hartree-Fock determinant. The first sum is over all singly excited determinants, with one occupied HF orbital substituted by a virtual orbital. The second sum is over all doubly excited determinants, with two HF orbitals substituted by two virtual orbitals. The $\mathrm{Cl}$ coefficients $C$ can be optimized by minimizing the energy functional under the constraint that the total wave function is normalized, similar to optimization of molecular orbital coefficients in the Hartree-Fock method. By using this approach it is possible to show that the coefficients $C$ must satisfy the eigenvalue equation

$$
\mathrm{HC}=E \mathrm{C}
$$

Here $\mathbf{C}$ is the vector of $\mathrm{CI}$ coefficients $C_{i}$. The eigenvalues $E$ are electronic energies of the ground and excited states. In the $C I$ basis the elements of the Hamiltonian $H$ are $H_{I J}=\left\langle\Psi_{l}|\mathbf{H}| \Psi_{J}\right\rangle$. The indices $I$ and $J$ stand for the Hartree-Fock or any excited determinants. If, in construction of Slater determinants, the canonical Hartree-Fock molecular orbitals are used, many matrix elements $H_{l J}$ are zero. For example, since the Hamiltonian is a twoelectron operator, all matrix elements between determinants with more than two distinct orbitals are zero. This property is a result of orthogonality of the Hartree-Fock orbitals. The matrix elements between the HF determinant and any singly excited determinant is zero due to Brillouin's theorem ${ }^{5}$. All non-zero $H_{I J}$ matrix elements can be expressed through twoelectron integrals over molecular orbitals. For example,

$$
\left\langle\Psi_{H F}|\mathbf{H}| \Psi_{i j}^{a b}\right\rangle=\left\langle\psi_{i} \psi_{j}\left|r_{12}^{-1}\right| \psi_{a} \psi_{b}\right\rangle-\left\langle\psi_{i} \psi_{j}\left|r_{12}^{-1}\right| \psi_{b} \psi_{a}\right\rangle
$$


The one- and two-electron integrals over molecular orbitals can be calculated from atomic integrals and molecular orbital coefficients.

$$
\begin{aligned}
& \left\langle\psi_{i}|\mathbf{h}| \psi_{j}\right\rangle=\sum_{\alpha}^{M} \sum_{\beta}^{M} c_{\alpha i} c_{\beta j}\left\langle\chi_{\alpha}|\mathbf{h}| \chi_{\beta}\right\rangle \\
& \left\langle\psi_{i} \psi_{j}\left|r^{-1}\right| \psi_{k} \psi_{i}\right\rangle=\sum_{\alpha}^{M} \sum_{\beta}^{M} \sum_{\gamma}^{M} \sum_{\delta}^{M} c_{\alpha i} c_{\beta j} c_{\gamma k} c_{\delta \delta}\left\langle\chi_{\alpha} \chi_{\beta}\left|r_{12}^{-1}\right| \chi_{\gamma} \chi_{\delta}\right\rangle
\end{aligned}
$$

If all determinants in (14) are used in a calculation the full configuration interaction (FCI) method gives the exact solution of the Schrödinger equation for a given one-electron basis. The number of determinants in expansion (14) grows factorially with the size of the one electron basis set (number of molecular orbitals) and with the number of electrons. As a result, $\mathrm{FCI}$ calculations are possible only for two or three atom systems. To make the $\mathrm{CI}$

method practical, the order of excitations can be restricted. The most popular method is configuration interaction with singles and doubles (CISD). In this method all coefficients $C_{I}$ in front of determinants with more then two excited electrons are neglected. One of the disadvantages of truncating the expansion (14) in this way is that any restricted $\mathrm{Cr}$ method is size inconsistent. In other words the sum of the energies of two separate fragments of the system is not equal to the energy of the whole system. As a result, as the size of a system increases, methods like CISD recover a smaller and smaller percentage of the correlation energy. The advantage of $\mathrm{Cl}$ methods is that they are variational, which means the calculated energy is always an upper bound to the exact energy.

\section{Møller-Plesset perturbation theory}

According to standard Rayleigh-Schrödinger perturbation theory, the first- and second-order correction to the electron energy of the ground state can be written as

$$
\begin{aligned}
E^{(1)} & =\left\langle\Psi_{0}\left|\mathbf{H}^{\prime}\right| \Psi_{0}\right\rangle \\
E^{(2)} & =\sum_{i=1}^{M} \frac{\left\langle\Psi_{0}\left|\mathbf{H}^{\prime}\right| \Psi_{i}\right\rangle\left\langle\Psi_{i}\left|\mathbf{H}^{\prime}\right| \Psi_{0}\right\rangle}{E_{0}-E_{i}}
\end{aligned}
$$


Here $\Psi_{t}$ and $E_{i}$ are eigenfunctions and eigenvalues of the unperturbed hamiltonian $\mathbf{H}_{0}$, and $\mathbf{H}^{\prime}$ is the perturbation. In Møller-Plesset perturbation theory ${ }^{6}$ the unperturbed hamiltonian is chosen as

$$
\mathbf{H}_{0}=\sum_{i=1}^{N} \mathbf{F}_{i}-\frac{1}{2} \sum_{i=1}^{N} \sum_{j=1}^{N}\left(J_{i j}-K_{i j}\right)
$$

The perturbation is the difference between the exact Hamiltonian $\mathbf{H}$ and the zeroth-order Hamiltonian $\mathbf{H}_{0}$.

$$
\mathbf{H}^{\prime}=\mathbf{H}-\mathbf{H}_{0}=\sum_{i=1}^{N} \sum_{j>i}^{N} r_{i j}^{-1}-\frac{1}{2} \sum_{i=1}^{N} \sum_{j=1}^{N}\left(J_{i j}-K_{i j}\right)=\sum_{i=1}^{N} \sum_{j>i}^{N} r_{i j}^{-1}-\frac{1}{2} \sum_{i=1}^{N} \sum_{j=1}^{N}\left\langle r_{i j}^{-1}\right\rangle
$$

Here $\left\langle r_{i j}^{-1}\right\rangle$ stands for an averaged (Hartree-Fock) electron interaction.

With this choice of $\mathbf{H}_{0}$ the zeroth-order wave function $\Psi_{0}$ is equal to the Hartree-Fock wave function, and the zeroth-order energy is equal to the Hartree-Fock energy.

$$
E^{(0)}=\left\langle\Psi_{0}\left|\mathbf{H}_{0}\right| \Psi_{0}\right\rangle=E_{H F}
$$

The first-order energy correction is zero.

$$
E^{(1)}=\left\langle\Psi_{0}\left|\mathbf{H}^{\prime}\right| \Psi_{0}\right\rangle=\frac{1}{2} \sum_{i=1}^{N} \sum_{j=1}^{N}\left\langle r_{i j}^{-1}\right\rangle-\frac{1}{2} \sum_{i=1}^{N} \sum_{j=1}^{N}\left\langle r_{i j}^{-1}\right\rangle=0
$$

The expression for the second-order Møller-Plesset energy correction contains only the Hartree-Fock determinant and doubly excited determinants. 


$$
E^{(2)}=\sum_{i<j}^{o c c} \sum_{a<b}^{p i r} \frac{\left\langle\Psi_{0}\left|\mathbf{H}^{\prime}\right| \Psi_{i j}^{a b}\right\rangle\left\langle\Psi_{i j}^{a b}|\mathbf{H}| \Psi_{0}\right\rangle}{E_{0}-E_{i j}^{a b}}
$$

This expression can be rewritten in terms of two-electron integrals over canonical molecular orbitals and its energies.

$$
E^{(2)}=\sum_{i<j}^{o c c} \sum_{a<b}^{\text {vir }} \frac{\left[\left\langle\psi_{i} \psi_{j}\left|r_{12}^{-1}\right| \psi_{a} \psi_{b}\right\rangle-\left\langle\psi_{i} \psi_{j}\left|r_{12}^{-1}\right| \psi_{b} \psi_{a}\right\rangle\right]^{2}}{\varepsilon_{i}+\varepsilon_{j}-\varepsilon_{a}-\varepsilon_{b}}
$$

In contrast to configuration interaction Møller-Plesset perturbation theory is size consistent, but not variational. The third-and fourth-order corrections to the energy can be calculated using MP3 and MP4 perturbation theory?

\section{E. Coupled Cluster method}

In contrast to the CI expansion (14) the coupled cluster wave function can be written $\mathrm{as}^{8}$

$$
\begin{aligned}
& \Phi_{c c}=\mathbf{e}^{\mathbf{T}} \Psi_{0} \\
& \mathbf{e}^{\mathbf{T}}=\mathbf{1}+\mathbf{T}+\frac{1}{2} \mathbf{T}^{2}+\frac{1}{6} \mathbf{T}^{3}+\ldots=\sum_{k=0}^{\infty} \frac{1}{k !} \mathbf{T}^{\mathbf{k}}
\end{aligned}
$$

where $\Psi_{0}$ is usually a Hartree-Fock wave function and $\mathbf{T}$ is a cluster operator. $\mathbf{T}$ can be written as sum of single, double etc. excitations.

$$
\begin{aligned}
& \mathbf{T}=\mathbf{T}_{1}+\mathbf{T}_{2}+\mathbf{T}_{3}+\ldots+\mathbf{T}_{\mathbf{N}} \\
& \mathbf{T}_{1} \Psi_{0}=\sum_{i}^{o c c} \sum_{a}^{v i r} t_{i}^{a} \Psi_{i}^{a} \\
& \mathbf{T}_{2} \Psi_{0}=\sum_{i<j}^{o c c} \sum_{a<b}^{v i r} t_{i j}^{a b} \Psi_{i j}^{a b}
\end{aligned}
$$


Amplitudes $t$ play the same role here as coefficients $C_{I}$ in configuration interaction expansion (14). The exponential operator can be written as

$$
\begin{aligned}
\mathbf{e}^{\mathrm{T}}= & 1+\mathrm{T}_{1}+\left(\mathrm{T}_{2}+\frac{1}{2} \mathrm{~T}_{1}^{2}\right)+\left(\mathrm{T}_{3}+\mathrm{T}_{2} \mathrm{~T}_{1}+\frac{1}{6} \mathrm{~T}_{1}^{3}\right)+ \\
& \left(\mathrm{T}_{4}+\mathrm{T}_{3} \mathrm{~T}_{1}+\frac{1}{2} \mathrm{~T}_{2}^{2}+\frac{1}{2} \mathrm{~T}_{2} \mathrm{~T}_{1}^{2}+\frac{1}{24} \mathrm{~T}_{1}^{4}\right)+\ldots
\end{aligned}
$$

In (27) each set of parentheses contains operators of the same order of excitations. Based on diagrammatic techniques of perturbation theory, the operators in each set of parentheses are divided into connected $\left(\mathbf{T}_{2}, \mathbf{T}_{3}, \mathbf{T}_{4}\right)$ and disconnected $\left(\mathbf{T}_{1}{ }^{2}, \mathbf{T}_{2} \mathbf{T}_{1}, \mathbf{T}_{1}{ }^{3}, \mathbf{T}_{3} \mathbf{T}_{1}, \mathbf{T}_{2}{ }^{2}\right.$ and $\left.\mathbf{T}_{2} \mathbf{T}_{1}{ }^{2}\right)$ operators. The connected operators of order $\mathrm{k}$ represent $\mathrm{k}$-electron interactions. The disconnected operators represent interactions between clusters of electrons. For example the interactions between two two-electron clusters are represented by the $\mathbf{T}_{2}{ }^{2}$ operator.

$$
E_{c c}=E_{0}+\sum_{i<j}^{o c c} \sum_{a<b}^{v i r}\left(t_{i j}^{a b}+t_{j}^{a} t_{j}^{b}-t_{i}^{b} t_{j}^{a}\right)\left(\left\langle\psi_{i} \psi_{j} \mid \psi_{a} \psi_{b}\right\rangle-\left\langle\psi_{i} \psi_{j} \mid \psi_{b} \psi_{a}\right\rangle\right)
$$

It is clear from (28) that $E_{c c}$ depends on singles and doubles amplitudes only. However, in order to calculate these amplitudes it is necessary to consider higher excitations. The set of nonlinear equations for $t_{i}^{a}, t_{i j}^{a b}$ can be obtained by multiplying the Schrödinger equation $H e^{T} \Psi_{0}=E e^{T} \Psi_{0}$ from the left by $\left\langle\Psi_{m}^{e}\right|,\left\langle\Psi_{m m}^{e f}\right|,\left\langle\Psi_{m m}^{e f g}\right| \ldots$. In general the knowledge of all amplitudes up to order $N$ (number of electrons in the system) are required to calculate $t_{i}^{a}, t_{i j}^{a b}$.

To get a manageable method the cluster operator $\mathbf{T}$ must be truncated. In the coupled cluster with singles and doubles (CCSD) approximation $\mathbf{T}=\mathbf{T}_{1}+\mathbf{T}_{2}$

$$
e^{T_{1}+T_{2}}=1+T_{1}+\left(T_{2}+\frac{1}{2} T_{1}^{2}\right)+\left(T_{2} T_{1}+\frac{1}{6} T_{1}^{3}\right)+\left(\frac{1}{2} T_{2}+\frac{1}{2} T_{2} T_{1}^{2}+\frac{1}{24} T_{1}^{4}\right)+\ldots
$$


The coupled cluster with singles, doubles and triples (CCSDT) method is too computationally expensive, but it is possible to include triply excited determinants by means of perturbation theory. $\operatorname{CSSD}(\mathrm{T})$ is one such method. The main advantage of the coupled cluster expansion (25) over configuration interaction (14) is that the former converges faster with increasing order of excitations. In addition, including the disconnected terms in the wave function expansion makes the coupled cluster approach size consistent. The main disadvantage of the coupled cluster method is that it is not variational.

\section{F. Methods based on multireference wave functions}

If electrons are strongly correlated, the single determinant Hartree-Fock wave function becomes a very poor approximation to the exact wave function. One type of strongly correlated system is molecules and clusters with partly broken chemical bonds. Some transition states of chemical reactions can be included in this class of strongly correlated systems. These are examples of near degeneracies of electronic states leading to significant diradical character.

If all electrons of the molecule or cluster are strongly correlated, all possible determinants of the $\mathrm{Cl}$ expansion must be included in the approximate wave function. Such a full configuration interaction ( $\mathrm{FCI}$ ) approach can only be applied to systems with up to a small number of electrons. Fortunately, in many cases only a small number of electrons in the system are strongly correlated. In such cases it is possible to separate a problem into two parts.

First, the small number of strongly correlated electrons can be treated with the multiconfigurational self-consistent field (MCSCF) method. In this method the so-called active space, consisting of a small number of electrons and a small number of molecular orbitals, is introduced. A CI type wave function is generated by exciting active electrons inside the active space in all possible ways; that is, a FCI within the chosen active space. Both $\mathrm{CI}$ coefficients $C_{l}$ and MO coefficients $c_{l}$ are optimized variationally. If all possible excitations inside the active space are considered, the method is called a fully optimized reaction space (FORS) ${ }^{9}$ or a complete active space self-consistent field (CASSCF). ${ }^{10}$ There are several different ways to reduce the number of determinants in a FORS wave function, by 
keeping only the most important ones. One of the most general method is optimized restricted multiple active space (ORMAS). ${ }^{11}$ This method allows one to choose several active spaces and restrict the number of excited determinants by specifying the maximal and minimal number of electrons in each active space.

In the second step the MCSCF wave function is used as a zeroth-order approximation for a multireference configurational interaction, perturbation theory or coupled cluster calculation. This allows one to account for the weakly (dynamically) correlated electrons.

The multireference configurational interaction method (MRCI) is very similar to the single reference $\mathrm{CI}$ method discussed earlier. In MRCI the additional excited determinants are generated from each determinant of the MCSCF wave function, whereas in the single reference $\mathrm{CI}$ method, excited determinants are generated from only one Hartree-Fock determinant. Similar to single reference CI, the order of excitation in MRCI has to be restricted. The multireference configurational interaction with singles and doubles (MRCISD) is the most popular type of MRCI method. The MRCI method is variational but not size consistent. It is possible to partly correct for the size consistency error by adding the renormalized Davidson (Q) correction ${ }^{12}$ to the MRCISD energy.

$$
Q=\frac{1-C_{0}^{2}}{C_{0}^{2}}\left(E_{M R C I S D}-E_{M C S C F}\right)
$$

Here $C_{0}$ is the contribution of the reference wave function (Hartree-Fock or MCSCF) to the total CISD or MRCICD wave function.

The MRCISD method is able to produce very high quality results if the MCSCF active space is large enough and chosen properly. However, MRCISD is very computationally demanding.

Second-order multireference perturbation theory (MRPT2) is more efficient than MRCISD and can be applied to much larger systems. Unfortunately, unlike single reference Möller-Plesset perturbation theory, there is no clear choice for a zeroth-order Hamiltonian in multireference perturbation theory. Therefore there are several different implementations of MRPT2. Most of the implementations are not size consistent, and none are variational. One 
of the serious problems of MRPT2 is that it often suffers from the so-called intruder state problem. Intruder states are excited MRPT2 determinants that are accidentally degenerate with one of the MCSCF determinants. Because of this degeneracy, the values of some denominators in MRPT2 can become very small, resulting in divergence of the second-order perturbation theory correction. The best way to attack the problem of intruder states is to include all intruder states in the MCSCF zeroth-order wave function. However, this can lead to a very large MCSCF active space and is not always possible. As an alternative to increasing the size of the active space, denominator shift techniques have been proposed ${ }^{13,14}$. Such techniques can be helpful in some cases, but since the results of such calculations often strongly depend on empirical shift parameters, they have to be used with caution.

The multireference coupled cluster approach is considered to be a very promising theory in quantum chemistry. However, to date the practical implementation has proved to be illusive. The main complication seems to be the intruder state problem, which is much more severe for multireference coupled cluster theory than for multireference perturbation theory.

\section{G. Density functional theory}

Density functional theory is based on the Hohenberg-Kohn theorem, which states that the energy of the ground state of an N-electron system is uniquely defined by the electron density $^{15}$. This allows one to replace a wave function, which is a function of $3 \mathrm{~N}$ coordinates, by the electron density, which is a function of only three space coordinates.

However, in order to take the advantage of this simplification it is necessary to know the energy as a functional of the electron density $\rho(x, y, z)$. Usually such a functional is written as a sum of the kinetic energy of the electrons $T[\rho]$, electron-nuclear interaction $E_{n e}[\rho]$, electron coulomb $J[\rho]$ and exchange-correlation $E_{x c}[\rho]$ interactions.

$$
\begin{aligned}
& E[\rho]=T[\rho]+E_{n e}[\rho]+J[\rho]+E_{x c}[\rho] \\
& E_{n e}[\rho]=\sum_{a} \int \frac{Z_{a} \rho(r)}{\left|R_{a}-r\right|} \\
& J[\rho]=\frac{1}{2} \iint \frac{\rho(r) \rho\left(r^{\prime}\right)}{\left|r-r^{\prime}\right|} d r d r^{\prime}
\end{aligned}
$$


The kinetic energy and exchange-correlation functional in general are unknown. However, by introducing Kohn-Sham 'molecular orbitals' the kinetic energy can be written as sum of one electron contributions. ${ }^{16}$

$$
T[\rho]=\sum_{i=1}^{N}\left\langle\psi_{i}\left|-\frac{1}{2} \nabla^{2}\right| \psi_{i}\right\rangle
$$

The electron density can be calculated from the Kohn-Sham orbitals as well.

$$
\rho=\sum_{i=1}^{N}\left|\psi_{i}\right|^{2}
$$

By minimizing the energy with respect to Kohn-Sham orbitals one can derive the KohnSham equations:

$$
\begin{aligned}
& \mathbf{h}_{k s} \psi_{i}=\varepsilon_{i} \psi_{i} \\
& \mathbf{h}_{k s}=-\frac{1}{2} \nabla^{2}+\mathbf{V}_{e f f} \\
& \mathbf{V}_{e f f}(\mathbf{r})=\mathbf{V}_{n e}(\mathbf{r})+\int \frac{\rho\left(\mathbf{r}^{\prime}\right)}{\left|\mathbf{r}-\mathbf{r}^{\prime}\right|} d \mathbf{r}^{\prime}+\mathbf{V}_{x c}(\mathbf{r})
\end{aligned}
$$

Here $\mathbf{h}_{k s}$ is one-electron Kohn-Sham operator, similar to the Fock operator in Hartree-Fock theory, $\varepsilon_{i}$ are the energies of the canonical Kohn-Sham orbitals, $\mathbf{V}_{n e}$ is the electron-nuclear interaction potential, and $\mathbf{V}_{x c}$ is the exchange-correlation potential.

The main problem of density functional methods comes from the fact that the exchange-correlation potential $\mathbf{V}_{x c}$ is unknown. Different density functional methods use different approximations for the exchange-correlation functional.

The simplest approximation for $\mathbf{V}_{x c}$ is called the Local Density Approximation (LDA). This approximation assumes that the local electron density can be written as the electron density of a homogeneous electron gas. In the more general Local Spin Density 
Approximation (LSDA) the density of the exchange energy is divided into two parts. One part depends on the electron density of all electrons with spin $\alpha$, the other part depends on the density of electrons with spin $\beta$.

The LSDA can be improved by considering the inhomogeneous instead of homogeneous electron gas. In this case the energy depends not only on the electron density but on derivatives of the electron density as well. Then the energy is represented as a functional of the electron density and its first derivative. This is called the Generalized Gradient Approximation (GGA). One of the commonly used GGA exchange functionals is the $\mathrm{B} 88$ functional. ${ }^{17}$ The Lee, Yang and Parr (LYP) functional $\quad{ }^{18}$ is a popular correlation GGA type functional.

In chemistry, the most popular class of density functionals is the so-called hybrid density functional. The exchange and correlation parts of these functionals are linear combinations of several different functionals. The exchange functionals usually include the non-local Hartree-Fock exchange. In the B3LYP functional the correlation energy is represented as a linear combination of LSDA, GGA and Hartree-Fock exchange functionals. The correlation energy is written as linear combination of LSDA and GGA correlation functionals. ${ }^{19}$

$$
E_{x c}^{B 3 L Y P}=(1-a) E_{x}^{L S D A}+a E_{x}^{H F}+b \Delta E_{x}^{B B 8}+E_{c}^{V W N}+c \Delta E_{c}^{L Y P}
$$

The parameters $a, b$ and $c$ are optimized to reproduce the experimental data for a set of molecular systems.

\section{Dissertation organization}

This dissertation contains seven chapters: chapters 2 through 6 are papers accepted, submitted to or in preparation for submission to peer reviewed journals. The present author is a primary contributor in all cases.

Chapter 2 is a study of the adsorption of oxygen molecules on small gold clusters. The binding energies of one and two oxygen molecules are calculated using MP2, CCSD(T) 
and DFT methods. It is shown that $\operatorname{CCSD}(\mathrm{T})$ binding energies are in good agreement with available experimental data. The DFT results are in qualitative disagreement with experimental data and results of $\operatorname{CCSD}(\mathrm{T})$ calculations.

Chapter 3 describes the study of reaction mechanisms of small gold clusters with molecular hydrogen. The barriers for hydrogen dissociation on neutral and anionic gold clusters are calculated with DFT, MP2 and CCSD(T) methods. The binding energies of one and two hydrogen molecules to small gold clusters are calculated.

Chapter 4 is a study of different isomers of $\mathrm{C}_{36}$ cluster. Using the CASSCF method and single and multireference perturbation theory it is shown that lowest energy isomer has $\mathrm{D}_{6 \mathrm{~h}}$ symmetry and significant diradical character. The high experimental stability of this isomer is explained by the presence of strong electron correlation.

Chapter 5 describes the study of the electronic ground and several excited states of $\mathrm{Ti}_{8} \mathrm{C}_{12}$ Met-Car cluster. The Jahn-Teller distortion from highly symmetrical $\mathrm{T}_{\mathrm{d}}$ structure to lower symmetry is discussed. The ionization potential of $\mathrm{Ti}_{8} \mathrm{C}_{12}$ is calculated using a number of different single and multireference quantum chemical methods.

Chapter 6 proposes a new method for numerical solution of the Schrödinger equation based on the diagonal part of the cumulant of the second-order density. The relations between the proposed method, the wave function approach and density functional theory are discussed.

Chapter 7 presents general conclusions of the work.

\section{References}

${ }^{1}$ D. J. Wales, Energy Landscapes. With Applications to Clusters, Biomolecules and Glasses (Cambridege university press) (2003).

${ }^{2}$ M. Born and R. Oppenheimer, Annalen der Physik (Berlin, Germany) 84, 457 (1927).

${ }^{3}$ C. C .J. Roothaan, Rev. Mod. Phys., 23, 69, (1951).

${ }^{4}$ G. G. Hall, Proc. R. Soc. (London), A205, 541, (1951).

${ }^{5}$ F. Jensen, Introduction to Computational Chemistry (John Willey \& Sons Ltd) (1999). 
${ }^{6}$ C. Møller, M.S. Plesset, Phys. Rev. 46, 618 (1934).

${ }^{7}$ R. Krishnan, J.A. Pople, Int. J. Quantum Chem. 14, 91 (1978).

${ }^{8}$ R.J. Bartlett, J. Phys. Chem. 93, 1697 (1989).

${ }^{9}$ K. Ruedenberg, M.W. Schmidt, M.M. Gilbert, S.T. Elbert, Chem. Phys. 71, 41 (1982); K. Ruedenberg, M.W. Schmidt, M.M. Gilbert, Chem. Phys. 71, 51 (1982); K. Ruedenberg, M.W. Schmidt, M.M. Gilbert, S.T. Elbert, Chem. Phys. 71, 65 (1982).

${ }^{10}$ B.O. Roos, Adv. Chem. Phys. 69, 399 (1987).

${ }^{11}$ J. Ivanic J. Chem. Phys. 119, 9364 (2003).

${ }^{12}$ S.L. Langhoff, E.R. Davidson, Int. J. Quantum Chem., 8, 61 (1974)

${ }^{13}$ B. O. Roos, K. Andersson, Chem. Phys. Lett. 245, 215 (1995).

${ }^{14}$ H. A. Witek, Y.-K. Choe, J. P. Finley, K. Hirao, J. Comp. Chem. 23, 957 (2002).

${ }^{15}$ P. Hohenberg and W. Kohn, Phys. Rev., 136, B864 (1964).

${ }^{16}$ W. Kohn, L.J. Sham, Phys. Rev., 140, A1133 (1965).

${ }^{17}$ A. D. Becke, Phys. Rev., B, 38, 3098 (1988).

${ }^{18}$ C. Lee, W. Yang and R. G. Parr, Phys. Rev. B, 37, 785 (1988).

${ }^{19}$ A. D. Becke, J. Chem. Phys., 98, 5648 (1993). 


\title{
CHAPTER 2. THE INTERACTION OF OXYGEN WITH SMALL GOLD CLUSTERS
}

Taken from a paper that has been published in the Journal of Chemical Physics 119, 2531 (2003).

Copyright 2003 American Institute of Physics.

Sergey A. Varganov, Ryan M. Olson, Mark S. Gordon and Horia Metiu

\begin{abstract}
Presented in this work are the results of a quantum chemical study of oxygen adsorption on small $\mathrm{Au}_{n}$ and $\mathrm{Au}_{n}^{-}(\mathrm{n}=2,3)$ clusters. Density functional theory (DFT), second order perturbation theory (MP2) and singles and doubles coupled cluster theory with perturbative triples $(\operatorname{CCSD}(T))$ methods have been used to determine the geometry and the binding energy of oxygen to $A u_{n}$. The multireference character of the wave functions has been studied using the complete active space self-consistent field (CASSCF) method. There is considerable disagreement between the oxygen binding energies provided by $\operatorname{CCSD}(\mathrm{T})$ calculations and those obtained with DFT. The disagreement is often qualitative, with DFT predicting strong bonds where $\operatorname{CCSD}(\mathrm{T})$ predicts no bonds or structures that are bonded but have energies that exceed those of the separated components. The $\operatorname{CCSD}(\mathrm{T})$ results are consistent with experimental measurements, while DFT calculations show, at a best, a qualitative agreement. Finally, the lack of a regular pattern in the size and the sign of the errors (as compared to CCSD(T)) is a disappointing feature of the DFT results for the present system: it is not possible to give a simple rule for correcting the DFT predictions (e.g. a useful rule would be that DFT predicts stronger binding of $\mathrm{O}_{2}$ by about $0.3 \mathrm{eV}$ ). It is likely that the errors in DFT appear not because of gold, but because oxygen binding to a metal cluster is a particularly difficult problem.
\end{abstract}




\section{Introduction}

Recent experiments have shown that small gold clusters deposited on a metal oxide surface are good oxidation catalysts ${ }^{1}$. This has stimulated theoretical work on oxygen adsorption in the gas-phase ${ }^{2}$, on oxide-supported $\mathrm{Au}_{\text {clusters }}{ }^{3}$ and on the mechanism of $\mathrm{CO}$ oxidation catalyzed by gold ${ }^{4}$. These papers used density functional theory (DFT) to calculate the binding energies and the activation energies needed for clarifying the reaction mechanism. Most catalytic systems have many electrons, making it challenging, but not impossible given new linear scaling methods ${ }^{5}$, to employ more reliable and more accurate methods.

When using DFT it is implicitly assumed that the calculated energies are sufficiently accurate for the task at hand. In most cases it is difficult to assess the-accuracy of DFT methods, since the experimental data needed for such tests is not available. Moreover, the theory is particularly valuable when it provides information about aspects of a reaction that cannot be obtained by experiments (e.g., the transition state, transient reaction intermediates). In such cases, the reliability of the results given by the DFT calculations must be determined without appeal to experiments.

One approach to testing the accuracy of DFT methods is to compare the results to those provided by better methods. To accomplish this one must study "model systems" that are small enough to allow the use of accurate methods, and large enough to contain all the significant aspects of the system of interest.

This article presents calculations of the binding energies of one or two oxygen molecules to $\mathrm{Au}_{2}$ and $\mathrm{Au}_{3}$ neutral and negatively charged clusters. To test the accuracy of the DFT calculations, energy differences based on the $\mathrm{B}^{2} \mathrm{LYP}^{6}$ and $\mathrm{PW} 91^{7}$ functionals are compared with those obtained using second order perturbation theory (MP2) ${ }^{8}$ and singles and doubles coupled cluster theory with perturbative triples $[\operatorname{CCSD}(\mathrm{T})]^{9}$, as well as with multireference methods. The multi-reference approaches ensure that a correct zeroth order wavefunction is used, while the perturbation and (especially) coupled cluster methods provide a correct accounting of dynamic correlation. 
In assessing the practical importance of the findings obtained in the present work, it is important to recognize that the key issue in catalysis studies is to understand the essence of the reaction mechanisms that lead to products via pathways that have the smallest activation energy. For example, in the case of $\mathrm{CO}$ oxidation one can envision two mechanisms: in one $\mathrm{O}_{2}$ dissociates and an $\mathrm{O}$ atom reacts with $\mathrm{CO}$ producing $\mathrm{CO}_{2}$; in the other $\mathrm{O}_{2}$ reacts with $\mathrm{CO}$ to form a carbonate and this reacts with $\mathrm{CO}$ to produce two $\mathrm{CO}_{2}$ molecules. One can decide between the two possibilities by finding which one has the lowest activation energy. The theoretical prediction may be considered to be acceptable if it is sufficiently accurate to tell which of the two activation energies is smaller. The same can be said about finding the most likely reaction intermediate from a set of candidates. DFT calculations could be very useful, if they are accurate enough for the tasks described earlier.

\section{II.Computational methods}

For all systems studied here the geometries were first determined using Hartree-Fock (HF) calculations. To ensure that the optimized geometries correspond to minima on their respective potential energy surfaces, the Hessian (matrix of energy second derivatives) was calculated and diagonalized. Hessians that are positive definite (no negative eigenvalues) correspond to local minima. Those with $\mathrm{n}$ negative eigenvalues correspond to $\mathrm{nth}$ order saddle points. The optimized HF geometries were used as the initial structures for DFT and MP2 geometry optimizations. The optimized MP2 geometries were then used for single point $\operatorname{CCSD}(\mathrm{T})$ calculations. The energy gradients for geometry optimizations were evaluated analytically for all HF, DFT and closed shell MP2 calculations. For open-shell MP2 geometry optimizations the gradients were calculated numerically.

To avoid spin contamination in the wavefunction, restricted open shell methods were used for all open shell calculations. For open shell second order perturbation theory the Zaveraged perturbation theory $\left(\mathrm{ZAPT}^{10}\right)$ version of restricted MP2 was used. The ZAPT and the restricted $\operatorname{RCCSD}(\mathrm{T})^{11}$ method are based on a restricted open shell $\mathrm{HF}$ wavefunction that is free of spin contamination. In the MP2 and $\operatorname{CCSD}(\mathrm{T})$ calculations the 1s-orbitals of 
oxygen and $5 \mathrm{~s}$ and $5 \mathrm{p}$ core orbitals of gold atoms were not included in the correlation part of the calculation.

To study the importance of nondynamic electron correlation, and to assess whether the single determinant-based methods provide an acceptable zeroth-order wave function in the small gold clusters, complete active space self-consistent field (CASSCF) ${ }^{12}$ and multireference second order perturbation theory $(\mathrm{MRMP} 2)^{13}$ calculations were carried out on $\mathrm{Au}_{2}$ and $\mathrm{Au}_{3}$ clusters.

The restricted DFT method with the B3LYP functional was used to compare with the

results of previous plane wave-based DFT calculations ${ }^{2}$ using the PW91 functional and with the MP2, MRMP2 and CCSD(T) calculations described above. The B3LYP functional consists of five functionals, namely Becke + Slater + HF exchange and LYP + VWN5 correlation.

In all calculations the effective core potential (ECP) with scalar relativistic corrections developed by Stevens et al. (SBKJC) ${ }^{14}$ was used for the gold atoms. This ECP retains 19 explicit electrons. The Gaussian contracted SBKJC basis set ${ }^{14}$ was augmented with one $f$ polarization (exponent $=0.89$ ) and one $s$ and one $p$ diffuse function (both exponents $=0.01$ ) centered on the gold atoms. The standard contracted $6-31+G^{*}$ basis set was centered on each oxygen atom. In all calculations spherical harmonic basis functions were used with five d-orbitals and seven f-orbitals. In order to study the effect of the contraction of the basis set on the computational results, calculations on $\mathrm{Au}_{2}$ were also carried out with uncontracted basis sets.

The GAMESS ${ }^{15}$ suite of programs was used for all calculations except for CCSD(T) which was done in MOLPRO ${ }^{16}$.

\section{Results and Discussion}

\section{A. Results for $\mathbf{A u}_{2}$}

In Table 1 we show the bond length, the binding energy and the vibrational frequency of $\mathrm{Au}_{2}$, calculated with a variety of methods and basis sets, as well as the experimental results. The starting basis set is the standard SBKJC ECP, augmented by a set of $f$ functions 
and a set of diffuse $s$ and $\mathrm{p}$ functions. Several observations about this table are pertinent: (a) As one might expect, compared with the experimental values, the bond distances predicted by HF are much too large, the dissociation energies much too small and the vibrational frequency too small. These observations are essentially independent of basis set. (b) The B3LYP results are considerably better than those obtained using $\mathrm{HF}$, they are essentially independent of basis set (suggesting that these results are converged with basis set), but the dissociation energy is still about $0.3 \mathrm{eV}$ too small, and the vibrational frequency is only marginally better than the HF value. (c) The MP2 results are in surprisingly good agreement with experiment, but this is clearly an artifact of the cancellation of basis set errors and level of theory errors since the agreement deteriorates as the basis set is improved. (d) The $\operatorname{CCSD}(\mathrm{T})$ results, with the smallest basis set, are already better than DFT and, unlike MP2, steadily improve as the basis set is improved. (e) Uncontracting the ECP valence basis set has little effect, and the same may be said of the multireference methods, CASSCF and MRMP2. Previous plane wave DFT calculations ${ }^{2}$ also predict reasonable dissociation energies, but these calculations are not expected to be as accurate as $\operatorname{CCSD}(\mathrm{T})$ in general.

Table 2 contains the values of vertical (anion has the neutral geometry) and adiabatic (optimal geometries for both neutral and anion) electron affinities (EA) of $\mathrm{Au}_{2}$ dimer, calculated with different methods using contracted and completely uncontracted basis sets. The experimental adiabatic EA has been estimated to be $1.94 \mathrm{eV}{ }^{17}$ Taylor et al. estimate the EA to be $2.02 \mathrm{eV}^{18}$. Mills, Gordon and Metiu ${ }^{2}$ calculate an adiabatic EA of $2.02 \mathrm{eV}$ using plane waves and the PW91 functional, and Hakkinen and Landman ${ }^{4(a)}$, using the PBE functional $^{19}$, predict $2.08 \mathrm{eV}$. Turning to Table 2 , it is clear that, as was noted for the results in Table 1, uncontracting the basis set does not significantly improve the results. Of course, this only expands the underlying s,p basis. The HF and CASSCF results are again unsatisfactory. All other methods are in much better agreement with experiment, although they all under-estimate the experimental value of $1.94 \mathrm{eV}$ by $0.2-0.4 \mathrm{eV}$. However, based on the results in Table 1, it is expected that an analogous basis set improvement, by adding more and higher 1 polarization functions, will bring the $\operatorname{CCSD}(\mathrm{T})$ results into much better agreement with experiment, while having little effect on the DFT predictions. 


\section{B. Results for $\mathrm{Au}_{3}$}

The $\mathrm{D}_{3 \mathrm{~h}}$ geometry of the $\mathrm{Au}_{3}$ cluster has one electron in the doubly degenerate $\mathrm{HOMO}$, so it is subject to Jahn-Teller distortion. The distorted $\mathrm{Au}_{3}$ cluster has $\mathrm{C}_{2 \mathrm{v}}$ symmetry and two possible electronic states, ${ }^{2} \mathrm{~A}_{1}$ and ${ }^{2} \mathrm{~B}_{2}$ (Figure 1). The energy relative to the ${ }^{2} \mathrm{~B}_{2}$ state and the geometry of the $\mathrm{Au}_{3}$ cluster are shown in Table 3 . According to experiment, ${ }^{20}$ the ground state of $\mathrm{Au}_{3}$ is ${ }^{2} \mathrm{~B}_{2}$, and the energy splitting between the ${ }^{2} \mathrm{~A}_{1}$ and the ${ }^{2} \mathrm{~B}_{2}$ states is very small. Wesendrup et $a l^{21}$ showed that even relativistic $\operatorname{CCSD}(\mathrm{T})$ calculations with large basis sets predict the ${ }^{2} A_{1}$ state to be the ground state with the energy splitting between it and the ${ }^{2} \mathrm{~B}_{2}$ state of $4.4 \mathrm{kcal} / \mathrm{mol}$. The same authors showed that telativistic MP2 calculations predict the ${ }^{2} \mathrm{~B}_{2}$ state to be $0.9 \mathrm{kcal} / \mathrm{mol}$ more stable than ${ }^{2} \mathrm{~A}_{1}$. The last result is consistent with the order of the states predicted by the multi-reference singles and doubles configuration interaction $\left(\mathrm{MRSDCl}^{22}\right)$ method $^{23}$.

The HF and MRMP2 methods predict, respectively, that the ${ }^{2} \mathrm{~A}_{1}$ state is $1.1 \mathrm{kcal} / \mathrm{mol}$ and $0.1 \mathrm{kcal} / \mathrm{mol}$ lower in energy than the ${ }^{2} \mathrm{~B}_{2}$ state. However, the CASSCF and MP2 methods predict that the ${ }^{2} \mathrm{~B}_{2}$ state is lower than ${ }^{2} \mathrm{~A}_{1}$ by 1.9 and $1.0 \mathrm{kcal} / \mathrm{mol}$, respectively. Interestingly, the B3LYP method and the plane-wave PW91 calculations predict no JahnTeller distortion: the linear ${ }^{2} \Sigma_{\mathrm{u}}{ }^{+}$is the lowest energy state. These results indicate that larger basis sets and consideration of the spin-orbit interaction among the states is required for an accurate prediction of the order of the $\mathrm{Au}_{3}$ electronic states. Since the energy difference between the ${ }^{2} \mathrm{~B}_{2}$ and ${ }^{2} \mathrm{~A}_{1}$ states is very small, in the following calculations of the binding energy of oxygen molecules to the $\mathrm{Au}_{3}$ cluster, it is assumed that ${ }^{2} \mathrm{~B}_{2}$ is the ground state.

\section{Results for $\mathrm{Au}_{3}{ }^{-}$}

All methods find that the $\mathrm{Au}_{3}{ }^{-}$cluster is linear, although local minima are also found in $C_{2 v}$ and $D_{3 h}$ symmetry. The energies of the $C_{2 v}{ }^{1} A_{1}$ and the $D_{3 h}{ }^{3} A_{1}$ states relative to linear ${ }^{1} \Sigma_{\mathrm{g}}{ }^{+}$are shown in Table 4. All methods predict that the ${ }^{3} \mathrm{~A}_{1}$ and ${ }^{1} \mathrm{~A}_{1}$ states are approximately 20 and $40 \mathrm{kcal} / \mathrm{mol}$, respectively, higher than ${ }^{1} \Sigma_{\mathrm{g}}{ }^{+}$. However, the methods that include dynamic correlation, MP2, CCSD(T) and MRMP2 all predict significantly shorter distances for all isomers than the other methods (Fig. 2). 


\section{Results for $\mathrm{Au}_{2} \mathrm{O}_{2}$ and $\mathrm{Au}_{2}\left(\mathrm{O}_{2}\right)_{2}$}

The binding energies of the first and second oxygen molecules to the neutral gold clusters were determined from

$$
\begin{gathered}
E_{b 1}=E\left[A u_{n}\right]+E\left[\mathrm{O}_{2}\right]-E\left[A u_{n} O_{2}\right] \\
E_{b 2}=E\left[A u_{n} O_{2}\right]+E\left[O_{2}\right]-E\left[A u_{n}\left(O_{2}\right)_{2}\right] .
\end{gathered}
$$

Here $n$ is the number of atoms in the specific gold cluster and $E[X]$ is the energy of the ground electronic state of the molecule $X$. The binding energies of the oxygen molecules to the negatively charged gold clusters were determined in the same way. Positive values for $E_{b 1}$ and $E_{b 2}$ indicate that adding the oxygen molecule actually lowers the energy of the cluster. A negative value suggests that an energy barrier separates the reactants and oxygenated cluster, since in this case the oxygenated cluster (although a minimum on the PES) is higher in energy than the separated reactants.

$\mathrm{Au}_{2} \mathrm{O}_{2}$ and $\mathrm{Au}_{2}\left(\mathrm{O}_{2}\right)_{2}$ are unstable at both the MP2 and HF levels of theory. In contrast, the previous DFT/PW91 calculation ${ }^{2}$ predicts a binding energy of $0.49 \mathrm{eV}$ for the first oxygen molecule and $0.29 \mathrm{eV}$ for the second. There is thus a substantial difference between the DFT and MP2 results.

\section{E. Results for $\mathrm{Au}_{2} \mathrm{O}_{2}{ }^{-}$and $\mathrm{Au}_{2}\left(\mathrm{O}_{2}\right)_{2}{ }^{-}$}

As shown in Table $5, \operatorname{CCSD}(\mathrm{T})$ theory gives $1.07 \mathrm{eV}$ for the binding energy of $\mathrm{O}_{2}$ to $\mathrm{Au}_{2}{ }^{-}$. This is slightly lower than the value predicted by the B3LYP calculations performed in the present work $(1.22 \mathrm{eV})$, the plane wave PW91 DFT calculations $(1.40 \mathrm{eV})^{2}$, and the plane wave PBE DFT calculations $(1.39 \mathrm{eV})^{4(\mathrm{a})}$. The experiments of Lee and Ervin $^{24}$ give $1.01 \pm 0.14 \mathrm{eV}$. Again, the binding energy predicted by density functional theory is too high. This is not entirely surprising, since it has been noted frequently that DFT tends to "overbind" $" 25$. If this error was systematic, one could "correct" for it and bring the results closer to reality. Unfortunately, as discussed below, the errors do not appear to be systematic overbinding. 
The $\operatorname{CCSD}(\mathrm{T})$ binding energy of the second $\mathrm{O}_{2}$ to form $\mathrm{Au}_{2}\left(\mathrm{O}_{2}\right)_{2}{ }^{2}$, is $-0.56 \mathrm{eV}$. The negative value indicates that this local minimum on the potential energy surface is higher in energy than the sum of the energies of the separated $\mathrm{O}_{2}+\mathrm{Au}_{2} \mathrm{O}_{2}{ }^{-}$. As noted above, this implies the presence of an intervening barrier for the removal of the second $\mathrm{O}_{2}$ molecule from the $\mathrm{Au}_{2}$ cluster. MP2 predicts a negative $\mathrm{E}_{2 \mathrm{~b}}$ that is even larger in magnitude. Plane wave DFT/PW91 calculations give a binding energy, $\mathrm{E}_{2 \mathrm{~b}}=0.71 \mathrm{eV}$, that is opposite in sign to the CCSD(T) and MP2 results. The discrepancy between the MP2 and CCSD(T) on one hand and the DFT calculation on the other, is substantial and again suggests that this level of theory tends to severely overbind oxygen to Au clusters.

Salisbury, Wallace and Whetten ${ }^{26}$ found that (a) negatively charged Au clusters will not adsorb $\mathrm{O}_{2}$ if the cluster has an even number of electrons, and (b) will adsorb one $\mathrm{O}_{2}$ molecule, but not a second one, if the cluster has an odd number of electrons. The MP2 and $\operatorname{CCSD}(\mathrm{T})$ results are in agreement with these experimental results, while the plane-wave DFT/PW91 results are not. The fast-flow reactor method used by Salisbury et al. has some limitations, as discussed recently by Wallace, Leavitt and Whetten ${ }^{27}$. However, the experimental results taken together with the MP2 and $\operatorname{CCSD}(\mathrm{T})$ calculations provide reasonable evidence that DFT does not describe the $\mathrm{Au}_{2}\left(\mathrm{O}_{2}\right)_{2}{ }^{-}$complex correctly.

Figure 3 illustrates the manner in which the electronic states of $\mathrm{Au}_{2}^{-}$, and two $\mathrm{O}_{2}$ molecules may correlate with the electronic states of $\mathrm{Au}_{2} \mathrm{O}_{2}^{-}$and $\mathrm{Au}_{2}\left(\mathrm{O}_{2}\right)_{2}{ }_{2}^{-}$. The ground state of $\mathrm{Au}_{2}\left(\mathrm{O}_{2}\right)_{2}{ }^{-}$is a quartet, whereas the ground states of $\mathrm{Au}_{2}{ }^{-}$and $\mathrm{Au}_{2} \mathrm{O}_{2}{ }^{-}$are doublets, and that of $\mathrm{O}_{2}$ is a triplet. Therefore, $\mathrm{Au}_{2}{ }^{-}$and $\mathrm{O}_{2}$ can spin couple to produce either a doublet or a quartet. The present calculations, as noted above, predict that the quartet is the ground state. So, there is no crossing of the doublet and quartet potential energy surfaces as the first oxygen molecule is added to the $\mathrm{Au}_{2}$ cluster. Adding the second $\mathrm{O}_{2}$ to the ground doublet state of $\mathrm{Au}_{2} \mathrm{O}_{2}{ }^{-}$can again produce either a doublet or a quartet. In this case, the calculations predict that the ground state of the product $\mathrm{Au}_{2}\left(\mathrm{O}_{2}\right)_{2}{ }^{-}$is the quartet. So, at some point between $\mathrm{Au}_{2} \mathrm{O}_{2}{ }^{-}+\mathrm{O}_{2}$ and $\mathrm{Au}_{2}\left(\mathrm{O}_{2}\right)_{2}{ }^{-}$the doublet and quartet potential energy surfaces cross. While an exhaustive study of these potential energy surfaces is beyond the scope of the 
present work, these results suggest that non-adiabatic interactions, such as spin-orbit coupling could be important in this region of the potential energy surfaces.

The fact that the energy of $\mathrm{Au}_{2}\left(\mathrm{O}_{2}\right)_{2}{ }^{-}$is higher than that of $\mathrm{Au}_{2} \mathrm{O}_{2}^{-}+\mathrm{O}_{2}$ indicates that at equilibrium the probability of observing this cluster with two oxygen molecules on it is small. This is consistent with the experiments of Salisbury et al., assuming the clusters are in thermal equilibrium in this experiment. The results of the DFT/PW91 calculations, according to which the second oxygen binds strongly, are compatible with the experiments only if it is valid to assume that the rate of the reaction $\mathrm{Au}_{2} \mathrm{O}_{2}{ }^{-}+\mathrm{O}_{2} \rightarrow \mathrm{Au}_{2}\left(\mathrm{O}_{2}\right)_{2}{ }^{-}$is so low that $\mathrm{Au}_{2}\left(\mathrm{O}_{2}\right)_{2}{ }^{-}$is not formed during the experiments. However, the most plausible conclusion is that the DFT calculations are in error.

These results for $\mathrm{Au}_{2}\left(\mathrm{O}_{2}\right)_{2}{ }^{-}$are relevant to the mechanism for $\mathrm{CO}$ oxidation by $\mathrm{Au}_{2}{ }^{-}$ cluster proposed by Hakkinen and Landman ${ }^{4(a)}$. Based on DFT calculations they proposed that catalysis takes place by adsorption of $\mathrm{O}_{2}$ and $\mathrm{CO}$ to form a carbonate. These authors did not consider the possibility that a second oxygen molecule may adsorb on the cluster, blocking $\mathrm{CO}$ adsorption. The DFT/PW91 calculations ${ }^{2}$, which give (probably incorrectly) the binding energy for the second oxygen to be $0.71 \mathrm{eV}$, indicate that the rates of adsorption and desorption of the second oxygen ought to be included as one of the steps in the kinetic scheme. The present MP2 and $\operatorname{CCSD}(T)$ calculations show, however, that $\mathrm{Au}_{2}\left(\mathrm{O}_{2}\right)_{2}{ }^{-}$is an unstable local minimum, so its formation will not affect $\mathrm{CO}$ oxidation. Another rationale for ignoring the adsorption of a second oxygen molecule in the $\mathrm{CO}$ oxidation process is the large predicted adsorption energy of $\mathrm{CO}^{4(\mathrm{a})}$.

\section{F. Oxygen binding to $\mathrm{Au}_{3}$}

The binding energies of one or two oxygen molecules to the $\mathrm{Au}_{3}$ cluster are given in Table 6. $\operatorname{CCSD}(\mathrm{T})$ predicts the first oxygen molecule barely binds to $\mathrm{Au}_{3}\left(\mathrm{E}_{\mathrm{bI}}=0.08 \mathrm{eV}\right)$ while DFT/PW91 predicts a binding energy of $0.90 \mathrm{eV}^{2}$. This degree of overbinding predicted by DFT is too large to be considered modest or systematically correctable. Both MP2 and HF predict a negative value for $\mathrm{E}_{\mathrm{bl}}$, while B3LYP predicts $+0.19 \mathrm{eV}$. It is interesting that in this case the Gaussian-based hybrid functional is in considerably better 
agreement with the benchmark $\operatorname{CCSD}(\mathrm{T})$ calculations than is the plane wave-based PW91 approach. Mills, Gordon and Metiu ${ }^{2}$ extended to neutral gold clusters, the rules proposed by Salisbury et $\mathrm{al}^{26}$. for adsorption of oxygen on negatively charged gold clusters. This extension, that was supported by the prior plane wave DFT/PW91 results ${ }^{2}$, postulates that oxygen binds weakly to neutral Au clusters having an even number of electrons, and more strongly to those having an odd number of electrons. The present $\operatorname{CCSD}(T)$ results are clearly incompatible with this conjecture.

The PW91 calculations predict that the binding energy of the second oxygen molecule to $\mathrm{Au}_{3}$ is fairly strong $(0.59 \mathrm{eV})$, whereas $\mathrm{CCSD}(\mathrm{T})$ predicts no binding. Clearly, the DFT/PW91 predictions for oxygen binding to $\mathrm{Au}_{3}$ are in strong disagreement with those of $\operatorname{CCSD}(\mathrm{T})$.

\section{G. Oxygen binding to $\mathrm{Au}_{3}{ }^{-}$}

As shown in Table 6, for $\mathrm{Au}_{3} \mathrm{O}_{2}{ }^{-} \mathrm{CCSD}(\mathrm{T})$ predicts a bound state whose energy exceeds that of the separated ground state $\mathrm{Au}_{3}{ }^{-}$and $\mathrm{O}_{2}$ by $0.38 \mathrm{eV}$; that is, $\mathrm{E}_{\mathrm{b}}$ is negative. MP2 predicts the binding energy to be even more negative. In contrast, DFT/PW91 calculation finds that the energy of the bound state of $\mathrm{Au}_{3} \mathrm{O}_{2}{ }^{-}$is $0.37 \mathrm{eV}$ below that of the fragments; that is $E_{b}$ is predicted to be positive. Therefore, the rule proposed by Salisbury et $\mathrm{al}^{26}$, that oxygen does not bind to the cluster $\mathrm{Au}_{3}{ }^{-}$, because it has an even number of electrons, is in agreement with the $\operatorname{CCSD}(\mathrm{T})$ and MP2 results. The DFT calculation is in quantitative disagreement with this rule, since it indicates binding. However, DFT/PW91 does predict that the binding of one $\mathrm{O}_{2}$ molecule to $\mathrm{Au}_{3}{ }^{-}$is much weaker than the binding to $\mathrm{Au}_{2}{ }^{-}$, in qualitative agreement with the rule of Salisbury et al.

Of the compounds studied here $\mathrm{Au}_{3}\left(\mathrm{O}_{2}\right)_{2}{ }^{-}$is the most surprising: the $\mathrm{CCSD}(\mathrm{T})$ binding energy of the second oxygen, $E_{\mathrm{b} 2}$, is $1.07 \mathrm{eV}$. This means that the energy

$$
\mathrm{E}_{\mathrm{b} 2}+\mathrm{E}_{\mathrm{bl}}=-\mathrm{E}\left(\mathrm{Au}_{3}\left(\mathrm{O}_{2}\right)_{2}{ }^{-}\right)+\mathrm{E}\left(\mathrm{Au}_{3}{ }^{-}\right)+2 \mathrm{E}\left(\mathrm{O}_{2}\right)
$$

for forming $\mathrm{Au}_{3}\left(\mathrm{O}_{2}\right)_{2}$ - from oxygen and $\mathrm{Au}_{3}{ }^{-}$is $1.07+(-0.38)=0.69$; that is, the complex is stable with respect to separated $2 \mathrm{O}_{2}$ and $\mathrm{Au}_{3}{ }^{-}$. 
This result seems to imply that the complex $\mathrm{Au}_{3}\left(\mathrm{O}_{2}\right)_{2}{ }^{-}$should be observed in the experiments, even though it is not seen. However, the absence of the complex in the experiments may be rationalized as follows. At the pressures used in the experiments three body collisions of $\mathrm{Au}_{3}{ }^{-}$with two oxygen molecules are very unlikely. Therefore, the complex must be formed by successive adsorption of oxygen molecules. However, the probability of forming $\mathrm{Au}_{3} \mathrm{O}_{2}{ }^{-}$is extremely low, since its energy exceeds that of separated $\mathrm{Au}_{3}{ }^{-}$and $\mathrm{O}_{2}$. For this reason the rate of forming $\mathrm{Au}_{3}\left(\mathrm{O}_{2}\right)_{2}{ }^{-}$should be small. Of course, the fact that $\mathrm{Au}_{3}\left(\mathrm{O}_{2}\right)_{2}{ }^{-}$is bound relative to its separated constituents means that $\mathrm{Au}_{3}\left(\mathrm{O}_{2}\right)_{2}{ }^{-}$must be present in an equilibrium mixture. However, the kinetics indicate that the time to reach equilibrium must be much longer than that used in the experiments of Salisbury et al ${ }^{26}$ Therefore, the absence of $\mathrm{Au}_{3}\left(\mathrm{O}_{2}\right)_{2}{ }^{-}$in the experiments is not in conflict with the $\mathrm{CCSD}(\mathrm{T})$ results. It does, however, disagree with the DFT/PW91 method, which predicts that clusters with one and two oxygen atoms on them should be observed. Although the experimental error is unknown, the $\operatorname{CCSD}(\mathrm{T})$ results are consistent with the available experimental evidence, and this is unlikely to change after a rigorous error analysis is performed.

\section{Summary}

It is assumed that the $\operatorname{CCSD}(\mathrm{T})$ calculations performed here represent the current state-of-the-art, against which the other levels of theory may be measured, especially when experimental data is lacking. Therefore, one concludes that density functional theory works well for the small neutral or negatively charged Au clusters considered here. In particular, DFT gives good results for the binding energy of $\mathrm{Au}_{2}$, the electron affinity of $\mathrm{Au}_{2}$ and the geometries of the larger clusters. However, DFT fails badly when it calculates the binding energies of one or two oxygen molecules to $\mathrm{Au}_{2}, \mathrm{Au}_{2}{ }^{-}, \mathrm{Au}_{3}$ and $\mathrm{Au}_{3}{ }^{-}$. One may argue that perhaps it is more difficult to describe the small clusters correctly, than the larger ones, and that the DFT results become better as the number of $\mathrm{Au}$ atoms is increased. However, the failure appears to be connected to the $\mathrm{O}_{2}$ molecule, which is notoriously difficult to describe correctly by quantum chemical calculations. Bonding of $\mathrm{O}_{2}$ to $\mathrm{Au}_{\mathrm{n}}$ involves a substantial 
charge transfer, and describing an $\mathrm{O}_{2}$ molecule with an excess of electronic charge is even more difficult. There is a chance that binding of other molecules to Au is less demanding. Calculations on the binding of $\mathrm{H}_{2}$ molecules to neutral and anionic Au clusters are now in progress.

The results presented here do not affect the mechanism proposed by Hakkinen and Landman for CO oxidation by $\mathrm{Au}_{2}{ }^{-}$. While the binding energy ( $1.07 \mathrm{ev}$ ) of $\mathrm{O}_{2}$ to $\mathrm{Au}_{2}{ }^{-}$found by $\operatorname{CCSD}(\mathrm{T})$ differs from that found by Hakkinen and Landman $(1.39 \mathrm{eV})$, this difference does not affect the qualitative conclusions drawn in their paper. Of course, only one step in their mechanism has been examined here.

It does not appear that the deviations of DFT from the $\operatorname{CCSD}(\mathrm{T})$ results are either systematic or predictable by some simple rule. Unfortunately, this means that no guidance can be provided regarding some reasonable corrections to be made when one uses DFT to study oxidation reactions.

The discrepancy between DFT and CCSD(T) is particularly dramatic because oxygen is involved. In a large number of cases DFT calculations agree well with experiments ${ }^{25}$. We have also found that the results of PW91 DFT and those of B3LYP agree well with CCSD(T) results for $\mathrm{H}$ adsorbed on Au clusters. ${ }^{30}$ Moreover, B3LYP calculations in Michael Bower's group $^{31}$ agree well with the binding energies of oxygen on small positive Ag clusters. In this case the binding energy of $\mathrm{O}_{2}$ is small and the oxygen molecule is not changes much upon binding, resulting in cancellation of the errors made on oxygen molecule. Because the behavior of DFT is not predictable, it is nevertheless important that cases of failure are carefully cataloged and documented along with the successes, to serve as a target for new and more accurate versions of DFT and to warn the users that in certain systems the DFT results should be used with caution.

\section{Acknowledgments}

The authors thank Dr. Michael Schmidt, Dr. Greg Mills and Dr. Dmitri Fedorov for helpful discussions. This work was supported by AFOSR through a DURINT grant. The calculations in this work were performed in part on an IBM workstation cluster made 
possible by grants from IBM in the form of a Shared University Research grant and the United State Department of Energy.

\section{References}

${ }^{1}$ M. Haruta, Catal. Today 36, 153 (1997); M. Haruta and M. Date, Appl. Cat. A: General, 222, 427 (2001); G. Bond and D. T. Thomson, Catal. Rev. Sci. Eng. 41, 319 (1999), G. C. Bond, Cat. Today, 72, 5, (2002).

${ }^{2}$ G. Mills, M. S. Gordon and H. Metiu, Chem. Phys. Lett. 359, 493 (2002).

${ }^{3}$ A. Sanchez, S. Abbet, U. Heiz, W. D. Shcneider, H. Hakkinen, R. N. Barnett and U. Landman, J. Phys. Chem. A 103, 9573 (1999).

${ }^{4}$ a) H. Hakkinen and U. Landman, J. Am. Chem. Soc. 123, 9704 (2001); b) N. Lopez and J. K. Norskov, J. Am. Chem. Soc. 124, 11262 (2002); c) Z-P Liu, P. Hu and A. Alavi, J. Am. Chem. Soc. (published on web, 2002)

${ }^{5}$ C. Hampel amd H.-J. Werner, J. Chem. Phys. 104, 6286 (1996); G. Hetzer, P. Pulay and H.J. Werner, Chem. Phys. Lett. 290, 143 (1998); M. Schütz, G. Hetzer, and H.-J. Werner, J. Chem. Phys. 111, 5691 (1999).

${ }^{6}$ A.D. Becke, J. Chem. Phys. 98, 5648 (1993); P.J. Stephens, F.J. Devlin, C.F. Chablowski, M.J. Frisch, J. Phys. Chem. 98, 11623 (1994); R.H. Hertwig, W. Koch, Chem. Phys. Lett. 268, 345 (1997).

${ }^{7}$ Y. Wang, J.P.Perdew, Phys.Rev. B44, 13298 (1991).

${ }^{8}$ C. Möller, M.S. Plesset, Phys. Rev. 46, 618 (1934).

${ }^{9}$ R.J. Bartlett, J. Phys. Chem., 93, 1697 (1989); C. Hampel, K. Peterson, and H.-J. Werner, Chem. Phys. Lett. 190, 1 (1992) and references therein. The program to compute the perturbative triples corrections has been developed by M.J.O. Deegan and P.J. Knowles (1992).

${ }^{10}$ T.J. Lee, D. Jayatilaka, Chem. Phys. Lett. 201, 1 (1993); T.J. Lee, A.P. Rendell, K.G. Dyall, D. Jayatilaka, J. Chem. Phys. 100, 7400 (1994).

${ }^{11}$ P.J. Knowles, C. Hampel, and H.-J. Werner, J. Chem. Phys. 99, 5219 (1993).

${ }^{12}$ B.O. Roos, Adv. Chem. Phys. 69, 399 (1987). 
${ }^{13}$ K. Hirao, Chem. Phys. Lett. 190, 374 (1992).

${ }^{14}$ W.J. Stevens, M. Krauss, H. Basch, P.G. Jasien, Can. J. Chem. 70, 612-630 (1992).

${ }^{15}$ M.W. Schmidt, K.K. Baldridge, J. A. Boatz, S.T. Elbert, M.S. Gordon, J.H. Jensen, S.

Koseki, N. Matsunaga, K.A. Nguyen, S.J. Su, T.L. Windus, M. Dupuis, J.A. Montgomery, J.

Comp.Chem. 14, 1347 (1993); G.D. Fletcher, M.W. Schmidt, M.S. Gordon, Adv. Chem.

Phys. 110, 267 (1999); G.D. Fletcher, M.W. Schmidt, B.M. Bode, M.S. Gordon, Comput.

Phys. Commun. 128, 190 (2000).

${ }^{16}$ MOLPRO is a package of ab-initio programs written by H.-J. Werner and P.J. Knowles, with contributions from R.D. Amos, A. Bernhardsson, A. Berning, P. Celani, D.L. Cooper, M.J.O. Deegan, A.J. Dobbyn, F. Eckert, C. Hampel, G. Hetzer, T. Korona, R. Lindh, A.W. Lloyd, S.J. McNicholas, F.R. Mandy, W. Meyer, M.E. Mura, A. Nicklass, P. Palmieri, R. Pitzer, G. Rauhut, M. Schutz, H. Stoll, A.J. Stone, R. Tarroni, and T. Thorsteinsson.

${ }^{17}$ J. Ho, K. M. Ervin and W. C. Lineberger, J. Chem. Phys. 93, 6987 (1990) and G. F. Gantefor, D. M. Cox and A. Kaldor, J. Chem. Phys. 93, 8395 (1990).

${ }^{18}$ K. J. Taylor, C. L. Pettiette-Hall, O. Chesnovsky, R. E. Smalley, J, Chem. Phys. 96, 3319 (1992).

${ }^{19}$ J. P. Perdew, K. Burke, M. Emzerhof, Phys. Rev. Lett. 77, 3865 (1996).

${ }^{20}$ J.A. Howard, R. Sutcliffe, and B. Mille, J. Chem. Soc. Chem. Comnum. 1449 (1983);

J.A. Howard, R. Sutcliffe, and B. Mille, Surf. Sci. 156, 214 (1985).

${ }^{21}$ R. Wesendrup, T. Hunt, and P. Schwerdtfeger, J. Chem. Phys. 112, 9356 (2000).

${ }^{22}$ H.-J. Werner and P.J. Knowles, J. Chem. Phys. 89, 5803 (1988); P.J. Knowles and H.-J. Werner, Chem. Phys. Lett. 145, 514 (1988).

${ }^{23}$ a) K. Balasubramanian, M.Z. Liao, Chem. Phys. 127, 313 (1988); b) K. Balasubramanian and K.K. Das, Chem. Phys. Lett. 186, 577 (1991).

${ }^{24} \mathrm{~T}$. H. Lee, Reactions and Bond Dissociation Energies of Bare and Ligated Copper Group Anions, Ph. D. thesis, University of Nevada, Reno, 1995.

${ }^{25}$ a) M. Scheffler and C. Stampfl, in Handbook of surface science, eds. K. Horn and M. Scheffler, Elsevier, Amsterdam, 1999; b) B. Hammer and J. K. Norskov, Adv. Cat. 45, 71 (2000).

${ }^{26}$ B.E. Salisbury, W.T. Wallace, and R.L. Whetten, Chem. Phys. 262, 131 (2000). 
${ }^{27}$ W. T. Wallace, A. J. Leavitt and R. L. Whetten, Chem. Phys. Lett. (to be published).

${ }^{28}$ B.A. Hess, U. Kaldor, J. Chem. Phys. 112, 1809 (2000).

${ }^{29}$ K. P. Huber, G. Herzberg, Molecular Spectra and Molecular Structure. IV. Constants of Diatomic Molecules, Van Nostrand, New York, 1979.

${ }^{30}$ S. A. Varganov, R. M. OIson, M. S. Gordon, and H. Metiu (unpublished).

${ }^{31}$ M. Manard and M. Bowers (private communication). 
Table 1. Bond distances $R(\AA)$, dissociation energies $D_{c}(e V)$ and vibrational frequencies $\omega_{c}(1 / \mathrm{cm})$ of the $\mathrm{Au}_{2}$ at different level of theory.

\begin{tabular}{|c|c|c|c|c|c|c|c|c|c|c|c|c|}
\hline & \multicolumn{3}{|c|}{ SBKJC(f)+sp } & \multicolumn{3}{|c|}{ uSBRJC $(f)+s p$} & \multicolumn{3}{|c|}{$45 B K J C(3 f)+5 p$} & \multicolumn{3}{|c|}{ uSBKJC $(3 f 2 g)+5 p$} \\
\hline & $\mathrm{R}(\mathrm{A})$ & $\mathrm{D}_{e}(\mathrm{eV})$ & $(1)(1 / \mathrm{cm})$ & $R(A)$ & $\mathrm{D}_{t}(\mathrm{eV})$ & $\omega_{e}(1 / \mathrm{cm})$ & $R(A)$ & $\mathrm{D}_{0}(\mathrm{eV})$ & $\omega_{0}(1 / \mathrm{cm})$ & $R(A)$ & $\mathrm{D}_{\mathrm{e}}(\mathrm{eV})$ & $\omega_{s}(1 / \mathrm{cm})$ \\
\hline$\overline{\mathrm{HF}}$ & 2.615 & 0.80 & 156 & 2.612 & 0.83 & 156 & 2.605 & 0.86 & 156 & 2.598 & 0.88 & 156 \\
\hline MP2 & $2.494^{\circ}$ & 2.24 & 190 & 2.472 & 2,28 & 194 & 2.469 & 2.35 & 196 & 2.448 & 2.47 & 200 \\
\hline $\operatorname{ccs} D$ & 2.539 & 1.89 & e & 2.521 & 1.89 & e & 2.527 & 1.87 & e & 2.509 & 1.94 & e \\
\hline $\operatorname{CcSD}(T)$ & 2.535 & 2.04 & 175 & 2.517 & 2.07 & e & 2.523 & 2.06 & $\mathrm{e}$ & 2.504 & 2.14 & e \\
\hline CASSCF $(2, Z)$ & 2.654 & 1.12 & 136 & e & e & e & e & e & $\mathrm{e}$ & e & e & e \\
\hline $\operatorname{MRMP2}(2,2)$ & 2.506 & 2.17 & 184 & 2.483 & 2.22 & e & 2.488 & 2.23 & e & 2.465 & 2.35 & e \\
\hline B3LYP & 2.562 & 1.98 & 167 & 2.560 & 1.97 & 167 & 2.547 & 2.07 & 167 & 2.539 & 2.03 & 168 \\
\hline PW-PBE & 2.54 & 2.22 & & & & & & & & & & \\
\hline$P W-P W 9]^{b}$ & 2.528 & 2.27 & & & & & & & & & & \\
\hline $\operatorname{cp}-\cos D(T)^{c}$ & 2.488 & 1.19 & 187 & & & & & & & & & \\
\hline Experimen $^{a}$ & 2.472 & 2.31 & 191 & & & & & & & & & \\
\hline
\end{tabular}

${ }^{\mathrm{a}} \mathrm{DFT}$ with plane-wave basis set and PBE functional [Ref. 4(a)].

${ }^{\mathrm{b}}$ DFT with plane-wave basis set and PW91 functional (Ref. 2).

${ }^{\circ}$ Counterpose-corrected CCSD(T) with all electron PJHN-4f2g1h1i basis set (Ref. 28).

${ }^{d}$ Experimental values (Ref. 28).

${ }^{e}$ Values are not available yet. 
Table 2. $\mathrm{Au}_{2}$ vertical and adiabatic electron affinities (eV) calculated with different levels of theory. Experimental values for the adiabatic electron affinity are $1.94 \mathrm{eV}$ (Ref 17) and 2.02 eV (Ref. 18). PW-PW91 (Ref. 2) and PW-PBE [Ref. 4(a)] values for adiabatic electron affinity are $2.02 \mathrm{eV}$ and $2.08 \mathrm{eV}$. Relativistic $\operatorname{CCSD}(\mathrm{T})$ with all electron (10s7p5d3f)/[10s6p5d3f] basis set value is $1.83 \mathrm{eV}$ (Ref. 21).

\begin{tabular}{l|lcccccc}
\hline & & HF & MP2 & CCSD(T) & CASSCF(2,2) & MRMP2 & B3LYP \\
\hline Contracted & Vertical & 0.58 & 1.43 & 1.46 & 0.33 & 1.49 & 1.66 \\
basis set & Adiabatic & 0.64 & 1.47 & 1.51 & 0.36 & 1.52 & 1.71 \\
\hline Uncontracted & Vertical & 0.59 & 1.48 & 1.51 & 0.34 & 1.54 & 1.68 \\
basis set & Adiabatic & 0.65 & 1.53 & 1.56 & 0.37 & 1.58 & 1.74 \\
\hline
\end{tabular}


Table 3. Bond distances $R(\AA)$, angles $\Theta\left(\right.$ degree) and energies $\Delta E(\mathrm{kcal} / \mathrm{mol})$ relative to ${ }^{2} \mathrm{~B}_{2}$ state of $\mathrm{Au}_{3}$ at different levels of theory.

\begin{tabular}{l|cc|ccc|cc}
\hline & \multicolumn{3}{|c|}{${ }^{2} \mathrm{~B}_{2}$} & \multicolumn{3}{c|}{${ }^{2} \mathrm{~A}_{1}$} & \multicolumn{2}{c}{${ }^{2} \Sigma^{+}{ }_{u}$} \\
\cline { 2 - 8 } & $\mathrm{R}$ & $\Theta$ & $\Delta \mathrm{E}$ & $\mathrm{R}$ & $\Theta$ & $\Delta \mathrm{E}$ & $\mathrm{R}$ \\
\hline $\mathrm{HF}$ & 2.711 & 71.8 & -1.1 & 2.983 & 52.6 & 1.4 & 2.701 \\
MP2 & 2.580 & 64.3 & 1.0 & 2.688 & 56.3 & 6.5 & 2.558 \\
CCSD(T) & $\mathrm{MP2}^{\mathrm{d}}$ & $\mathrm{MP2}$ & 0.3 & $\mathrm{MP2}$ & $\mathrm{MP2}$ & 5.0 & $\mathrm{MP2}$ \\
CASSCF(3,3) & 2.749 & 143.5 & 1.9 & 3.031 & $52.2 \mathrm{TS}$ & 0.0 & 2.755 \\
MRMP2 & 2.591 & 64.9 & -0.1 & 2.681 & 56.8 & 34.8 & 2.629 \\
RB3LYP & 2.649 & 68.6 & 0.7 & 2.945 & 52.2 & -0.5 & 2.628 \\
UB3LYP & 2.650 & 68.5 & 0.7 & 2.793 & 55.7 & -0.5 & 2.630 \\
\hline R-CCSD(T) & 2.607 & 65.4 & -4.4 & 2.723 & 56.2 & 58.4 & 2.573 \\
R-MP2 & 2.552 & 65.1 & 0.9 & 2.665 & 56.1 & 3.2 & 2.517 \\
MRSDCI $^{\mathrm{a}}$ & 2.60 & 65.7 & 0.6 & 2.72 & 56.4 & --- & - \\
\hline
\end{tabular}

"Relativistic CCSD(T) and MP2 with all electron (10s7p5d3f)/[10s6p5d3f] basis set(Ref. 21).

${ }^{\mathrm{b}}$ Multi-reference single and double configuration interaction with Davidson correction [Ref. 23(a)].

This is a transition state on the potential energy surface.

${ }^{\mathrm{d} M P 2}$ geometries. 
Table 4. Bond distances $R(\AA)$, angles $\Theta$ (degree) and energies $\Delta E(\mathrm{kcal} / \mathrm{mol})$ relative to $\mathrm{Au}_{3}{ }^{-}$ ${ }^{1} \Sigma_{\mathrm{g}}^{+}$state at different levels of theory.

\begin{tabular}{|c|c|c|c|c|c|c|}
\hline & ${ }^{7} \Sigma_{g^{+}}$ & \multicolumn{3}{|c|}{$\mathrm{C}_{2 \mathrm{v}}\left(\mathrm{A}_{1}\right)$} & \multicolumn{2}{|c|}{$D_{3 h}\left(A_{A_{1}}\right)$} \\
\hline & $\mathbf{R}$ & $\Delta \mathrm{E}$ & $\mathbf{R}$ & $\Theta$ & $\Delta \mathrm{E}$ & $\mathrm{R}$ \\
\hline$\overline{\mathrm{HF}}$ & 2.711 & 37.1 & 3.326 & 46.2 & 18.8 & 2.880 \\
\hline MP2 & 2.550 & 37.8 & 2.785 & 53.6 & 23.4 & 2.664 \\
\hline $\operatorname{CCSD}(T)$ & $M P 2^{\mathrm{b}}$ & 36.0 & MP2 & MP2 & 24.4 & MP2 \\
\hline $\operatorname{CASSCF}(3,4)$ & 2.718 & 40.5 & MP2 & MP2 & 24.2 & 2.880 \\
\hline MRMP2 & 2.559 & 38.8 & MP2 & MP2 & 18.4 & MP2 \\
\hline B3LYP & 2.631 & 38.9 & 2.945 & 52.2 & 28.4 & 2.78 \\
\hline$R-\operatorname{CcSD}(T)^{a}$ & 2.573 & 41.3 & 2.844 & 53.4 & 29.1 & 2.717 \\
\hline R-MP2 ${ }^{\mathrm{a}}$ & 2.511 & 45.4 & 2.792 & 52.7 & 30.0 & 2.647 \\
\hline
\end{tabular}

${ }^{\mathrm{a}}$ Relativistic $\operatorname{CCSD}(\mathrm{T})$ and MP2 with all electron $(10 \mathrm{~s} 7 \mathrm{p} 5 \mathrm{~d} 3 \mathrm{f}) /[10 \mathrm{~s} 6 \mathrm{p} 5 \mathrm{~d} 3 \mathrm{f}]$ basis set (Ref. 21).

${ }^{\mathrm{b}} \mathrm{MP2}$ geometries. 
Table 5. $\mathrm{Au}_{2}{ }^{-}$. Binding energies (eV) for the first, $\mathrm{E}_{\mathrm{b} 1}$, and the second, $\mathrm{E}_{\mathrm{b} 2}, \mathrm{O}_{2}$

\begin{tabular}{lccccc}
\hline & $H F$ & $M P 2$ & $\operatorname{CCSD}(T)$ & B3LYP & PW-PW91 \\
\hline$E_{b 1}$ & 0.69 & 0.51 & 1.07 & 1.22 & 1.40 \\
$E_{b 2}$ & -0.52 & -1.57 & -0.56 & $b$ & 0.71 \\
\hline
\end{tabular}

${ }^{a}$ DFT with plane-wave basis set and PW91 functional (Ref. 2).

${ }^{\mathrm{b}}$ The B3LYP calculation for $\mathrm{E}_{\mathrm{b} 2}$ did not converge. 
Table 6. $\mathrm{Au}_{3} / \mathrm{Au}_{3}{ }^{-}$Binding energies (eV) for the first, $\mathrm{E}_{\mathrm{b} 1}$, and the second, $\mathrm{E}_{\mathrm{b} 2}, \mathrm{O}_{2}$. Unstable means that molecule does not bind. Because of this CCSD(T) cannot be performed. B3LYP converged only for $\mathrm{Au}_{3}, \mathrm{E}_{\mathrm{b} 1}$.

\begin{tabular}{lccccc}
\hline & $\mathrm{HF}$ & $\mathrm{MP2}$ & $\mathrm{CCSD}(\mathrm{T})$ & $\mathrm{B3LYP}$ & PW-PW91 $^{\mathrm{a}}$ \\
\hline $\mathrm{Au}_{3}{ }^{-}, \mathrm{E}_{\mathrm{b} 1}$ & -0.38 & -1.10 & -0.38 & & 0.37 \\
$\mathrm{Au}_{3}, \mathrm{E}_{\mathrm{b} 2}$ & 0.57 & 0.52 & 1.07 & & 0.70 \\
$\mathrm{Au}_{3}, \mathrm{E}_{\mathrm{b} 1}$ & -0.14 & -0.68 & 0.08 & 0.19 & 0.90 \\
$\mathrm{Au}_{3}, \mathrm{E}_{\mathrm{b} 2}$ & unstable & unstable & --- & & 0.59 \\
\hline
\end{tabular}

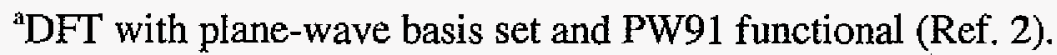




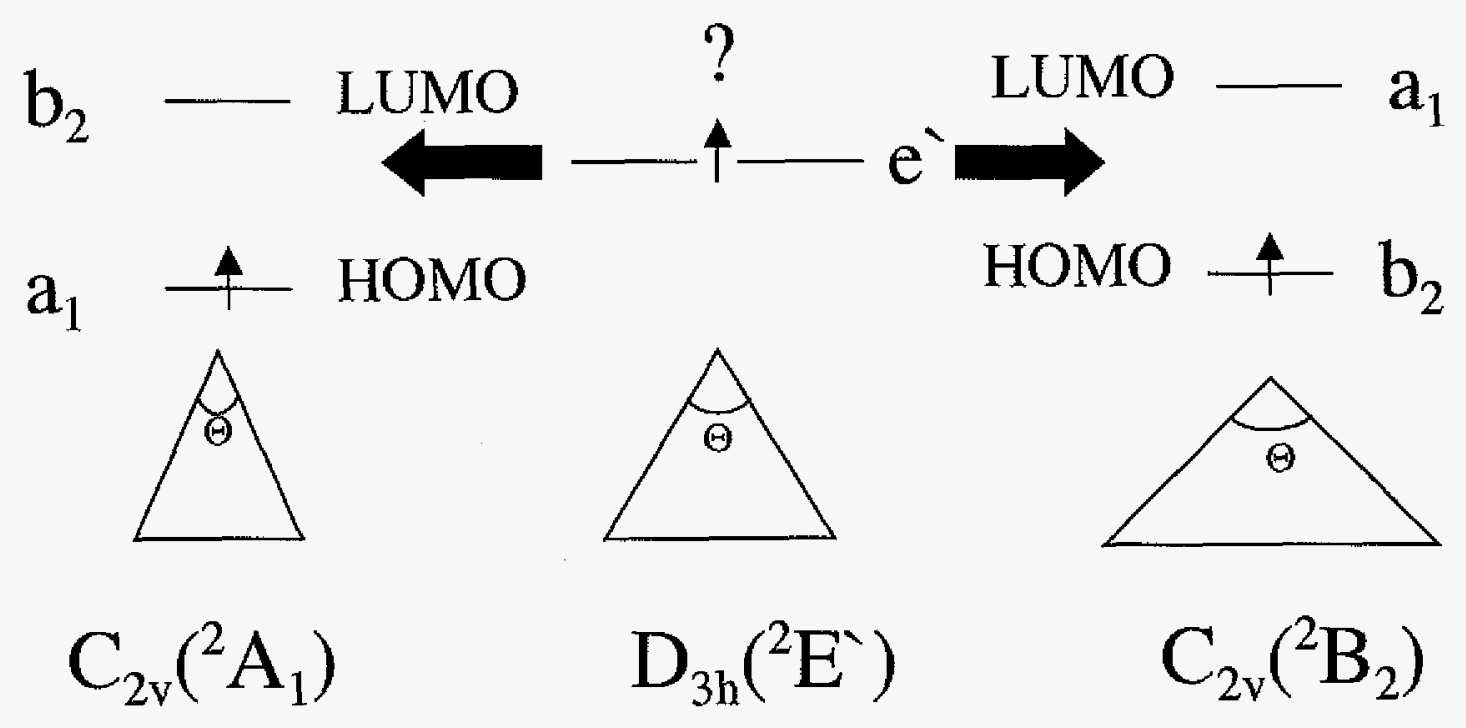

Figure 1. Jahn-Teller distortion in $\mathrm{Au}_{3}$ cluster. Highest occupied (HOMO) and lowest unoccupied (LUMO) molecular orbitals of $\mathrm{Au}_{3}$ are shown. 
bond lengths are in $\AA$, angles are in degrees

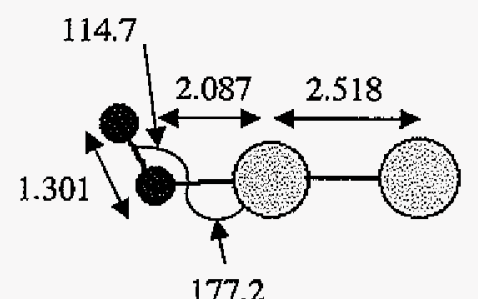

$\mathrm{Au}_{2} \mathrm{O}_{2}{ }^{2} \mathrm{~A} "$

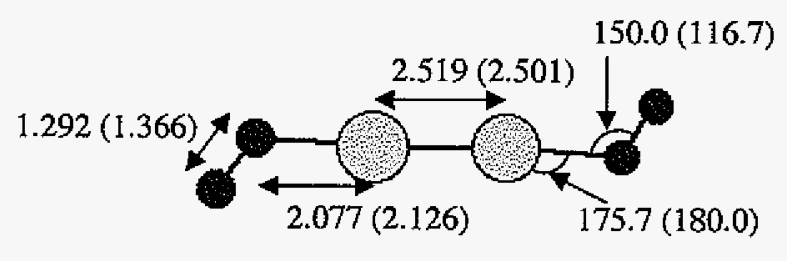

$$
\mathrm{Au}_{2} \mathrm{O}_{4}^{-4} \mathrm{~B}_{\mathrm{u}}\left({ }^{2} \mathrm{~B}_{\mathrm{g}}\right)
$$

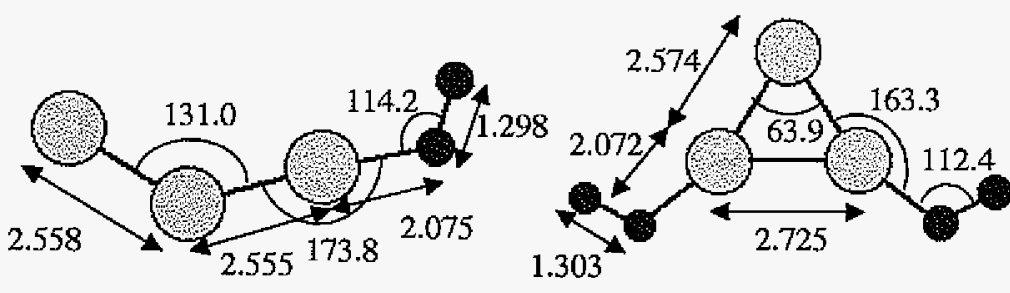

$$
\mathrm{Au}_{3} \mathrm{O}_{2}^{-3} \mathrm{~A}^{\prime \prime}
$$

$$
\mathrm{Au}_{3} \mathrm{O}_{4}{ }^{-3} \mathrm{~B}_{1}
$$

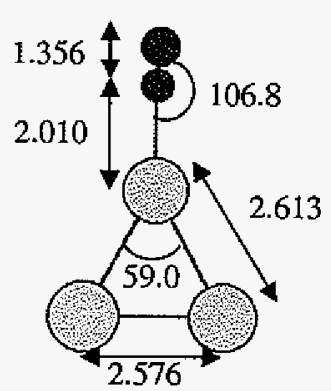

$\mathrm{Au}_{3} \mathrm{O}_{2}{ }^{2} \mathrm{~A}^{\prime}$

Figure 2. MP2 optimized geometries of the lowest electronic states of $\mathrm{Au}_{2} \mathrm{O}_{2}, \mathrm{Au}_{2} \mathrm{O}_{4}, \mathrm{Au}_{2} \mathrm{O}_{2}^{-}$ and $\mathrm{Au}_{2} \mathrm{O}_{4}{ }^{-}$complexes. For $\mathrm{Au}_{2} \mathrm{O}_{2}$ the geometry of the lowest quartet and doublet states are shown. 


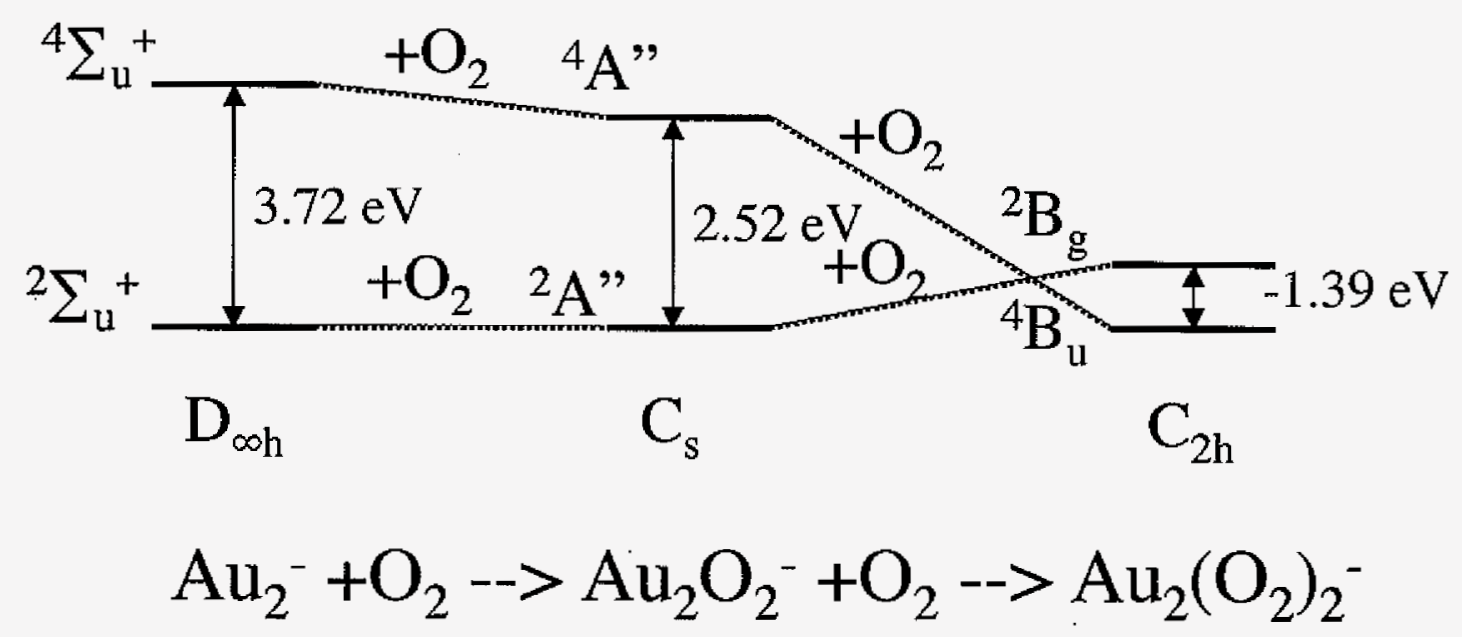

Energies were calculated at $\operatorname{CCSD}(\mathrm{T})$ level of theory

Figure 3. Correlation of the electronic states of $\mathrm{Au}_{2}{ }^{-}$, and two $\mathrm{O}_{2}$ molecules with the electronic states of $\mathrm{Au}_{2} \mathrm{O}_{2}{ }^{-}$and $\mathrm{Au}_{2}\left(\mathrm{O}_{2}\right)_{2}{ }^{-}$. 


\title{
CHAPTER 3. A STUDY OF THE REACTIONS OF MOLECULAR HYDROGEN WITH SMALL GOLD CLUSTERS
}

Taken from a paper that has been published in the Journal of Chemical Physics 120, 5169, (2004).

Copyright 2004 American Institute of Physics.

\author{
Sergey A. Varganov, Ryan M. Olson and Mark S. Gordon, \\ Greg Mills and Horia Metiu
}

\begin{abstract}
This work presents a study of reactions between neutral and negatively charged $\mathrm{Au}_{\mathrm{n}}$ clusters $(\mathrm{n}=2,3)$ and molecular hydrogen. The binding energies of the first and second hydrogen molecule to the gold clusters were determined using density functional theory (DFT), second order perturbation theory (MP2) and coupled cluster (CCSD(T)) methods. It is found that molecular hydrogen easily binds to neutral $\mathrm{Au}_{2}$ and $\mathrm{Au}_{3}$ clusters with binding energies of $0.55 \mathrm{eV}$ and $0.71 \mathrm{eV}$, respectively. The barriers to $\mathrm{H}_{2}$ dissociation on these clusters with respect to $\mathrm{Au}_{\mathrm{n}} \mathrm{H}_{2}$ complexes are $1.10 \mathrm{eV}$ and $0.59 \mathrm{eV}$ for $\mathrm{n}=2$ and 3. Although negatively charged $\mathrm{Au}_{\mathrm{n}}{ }^{-}$clusters do not bind molecular hydrogen, $\mathrm{H}_{2}$ dissociation can occur with energy barriers of $0.93 \mathrm{eV}$ for $\mathrm{Au}_{2}{ }^{-}$and $1.39 \mathrm{eV}$ for $\mathrm{Au}_{3}{ }^{-}$. The energies of the $\mathrm{Au}_{2} \mathrm{H}_{2}{ }^{-}$and $\mathrm{Au}_{3} \mathrm{H}_{2}{ }^{-}$ complexes with dissociated hydrogen molecules are lower than the energies of $\mathrm{Au}_{2}{ }^{-}+\mathrm{H}_{2}$ and $\mathrm{Au}_{3}{ }^{-}+\mathrm{H}_{2}$ by $0.49 \mathrm{eV}$ and $0.96 \mathrm{eV}$, respectively. There is satisfactory agreement between the DFT and CCSD(T) results for binding energies, but the agreement is not as good for barrier heights.
\end{abstract}

\section{Introduction}

This paper is concerned with the adsorption and the dissociation of $\mathrm{H}_{2}$ on very small, neutral and negatively charged $\mathrm{Au}_{\mathrm{n}}$ clusters ( $\left.\mathrm{n}=2,3\right)$. Its main purpose is to study hydrogen 
chemistry on these clusters and to test the accuracy of the density functional method by comparing its results to those obtained by using the coupled cluster method $\operatorname{CCSD}(T)^{1}$.

The interest in hydrogen chemistry on gold clusters has been stimulated by the recent discovery that supported gold clusters catalyze the hydrogenation of ethylene, 1,3-butadiene, 1-butene $\mathrm{e}^{2}$, acrolein ${ }^{3}$, and the partial hydrogenation of acetylene to ethylene $\mathrm{e}^{4}$.

Even more interesting is the partial oxidation of propylene to propylene oxide, by a mixture of hydrogen and oxygen, which is catalyzed by small Au clusters supported on $\mathrm{TiO}_{2}{ }^{5}$ It is rather unusual that an oxidation reaction requires the presence of hydrogen, and the reason for this is not yet understood. About $30 \%$ of the hydrogen is converted to water, which is a waste of an expensive feedstock. Therefore, work that furthers our understanding of the role of hydrogen in this process may have practical consequences. Other reactions catalyzed by small gold clusters are described in several excellent review articles.

Most electronic structure calculations in the fields of catalysis or surface science are performed by using density functional theory (DFT). This theory, which has had notable successes in explaining a variety of observations ${ }^{6}$, offers no a priori criterion for assessing the magnitude of the errors. These can be established only by comparison with experiments and/or higher level calculations. It is therefore prudent to test the accuracy of DFT, for each new system, before embarking on extensive predictive calculations on large systems of practical interest. This is especially important since we have recently illustrated that DFT calculations do not account, qualitatively or quantitatively, for the adsorption of molecular oxygen on small gold clusters?

Calculations and experiments on small gas-phase clusters play a central role in such comparisons. Negatively charged clusters are of interest because experiments on them are more readily available than on neutral clusters. Furthermore, they may play a role in catalysis by supported clusters, since the Au clusters tend to bind on oxygen vacancies ${ }^{8}$ that are electron rich ${ }^{9}$ and are chemically active ${ }^{10}$. A very interesting example of the activation of a small gold cluster by contact with an oxygen vacancy on $\mathrm{MgO}$ is provided by the beautiful work of Sanchez et $\mathrm{al}^{11}$.

Finally, note that most industrial catalysts use large clusters, containing thousands of $\mathrm{Au}$ atoms. It is not clear whether such large clusters are necessary, and small clusters may 
present an interesting alternative, especially since their properties change markedly when the number of atoms is changed or when they are electrically charged or alloyed. Thus, they offer more opportunities for performance optimization than very large clusters. Unfortunately, their use has been prevented so far by their propensity to coarsen and future uses of small clusters will have to solve this problem.

\section{Computational methods}

The geometries determined using Hartree-Fock (HF) calculations were used as the initial structures for DFT and MP2 ${ }^{12}$ geometry optimizations. If, during HF geometry optimization $\mathrm{H}_{2}$ did not bind to a Au cluster the optimization process was repeated with DFT and MP2 using a reasonable initial guess for the geometry. To ensure that the optimized geometries correspond to minima or transition states on their respective potential energy surfaces, the Hessian (matrix of energy second derivatives) was calculated and diagonalized. A Hessian with $\mathbf{n}$ negative eigenvalues corresponds to an $\mathrm{n}$-th order saddle point. Therefore, a zeroth order saddle point is a minimum on the potential energy surface, and a first order saddle point is a transition state. Since even relatively simple potential energy surfaces can have several minima and transition states, the intrinsic reaction path (IRC) method ${ }^{13}$, using the second order Gonzalez-Schlegel algorithm ${ }^{14}$ was used to connect the transition states with minima. This technique guarantees that one has found the correct transition state connecting two known minima. The optimized MP2 geometries were then used for single point $\operatorname{CCSD}(\mathrm{T})$ energy calculations. The energy gradients for geometry optimizations were evaluated analytically for all HF, DFT and closed shell MP2 calculations. For open-shell MP2 geometry optimizations the gradients were calculated numerically.

To avoid spin contamination in the wavefunction, restricted open shell methods were used for all open shell calculations. For open shell second order perturbation theory the Zaveraged perturbation theory $\left(\mathrm{ZAPT}^{15}\right)$ version of restricted MP2 was used. The ZAPT and the restricted $\operatorname{RCCSD}(\mathrm{T})^{16}$ method are based on a restricted open shell HF wavefunction that is free of spin contamination. In the MP2 and $\operatorname{CCSD}(\mathrm{T})$ calculations the $1 \mathrm{~s}$-orbitals of oxygen and $5 \mathrm{~s}$ and $5 \mathrm{p}$ core orbitals of gold atoms were not included in the correlation part of the calculation. 
The restricted DFT method with the B3LYP ${ }^{17}$ functional was used to compare with the results of plane wave-based DFT calculations and with the MP2 and $\operatorname{CCSD}(T)$ calculations described above. The B3LYP functional consists of five functionals, namely Becke + Slater + HF exchange and LYP + VWN5 correlation.

In all calculations the effective core potential (ECP) with scalar relativistic corrections developed by Stevens et al. (SBKJC) ${ }^{18}$ was used for the gold atoms. This ECP retains 19 explicit electrons. For geometry optimizations and Hessian calculations the basis set defined as "small" was used. This basis set consists of the Gaussian contracted SBKJC basis set ${ }^{17}$ augmented with one set of $f$ polarization functions (exponent $=0.89$ ) and one $s$ and one set of $p$ diffuse functions (both exponents $=0.01$ ) centered on the gold atoms. The standard contracted 6-31++G** (Ref. 19) basis set was centered on each hydrogen atom. A "large" basis set was used for single point calculations on geometries optimized with the small basis set. The large basis set consists of the completely uncontracted SBKJC basis set augmented with a $3 \mathrm{f} / 2 \mathrm{~g}$ set of polarization functions ( $\mathrm{f}$ function exponents $=2.00,0.84,0.3 \mathrm{I}$; $\mathrm{g}$ function exponents $=1.90,0.69$ ) and one $\mathrm{s}$ and one set of $\mathrm{p}$ diffuse functions (both exponents $=0.01$ ). The exponents of the polarization functions were optimized using MP2 calculations for atomic Au. The standard aug-cc-pVTZ $Z^{20}$ basis set was centered on each hydrogen atom. In all calculations spherical harmonic basis functions were used with five dorbitals and seven f-orbitals.

For plane wave DFT calculations we used the Perdew-Wang (PW91) ${ }^{21}$ functional, and the revised Perdew-Burke-Ernzerhof (rPBE) exchange-correlation functional ${ }^{22}$. The projector-augmented wavefunction pseudopotentials, ${ }^{23}$ as implemented in the VASP program ${ }^{24}$ was used. These pseudopotentials retain 11 explicit electrons for gold atom. The plane wave cutoff energy was $250 \mathrm{eV}$. The fictitious effect of the dipole and monopole (for anionic systems) moments of periodic images were removed by the method of Makov and Payne ${ }^{25}$.

The GAMESS ${ }^{26}$ suite of programs was used for all calculations except for CCSD(T) and plane wave density functional calculations which were done using MOLPRO ${ }^{27}$ and VASP, ${ }^{24}$ respectively. 


\section{Results and Discussion}

Results for $\mathrm{Au}_{2}$ : In order to estimate the reliability of the methods and basis sets used in this paper, test calculations were carried out on $\mathrm{Au}_{2}$. The values of the bond length, dissociation energy and vibrational frequency, calculated with different methods and basis sets, are presented in Table 1 . The HF method is clearly unable to correctly predict the dissociation energy of the gold dimer. The predicted HF dissociation energy is only $\sim$ onethird of the experimental value. The bond distance and vibrational frequency deviate significantly from the experimental values as well. These poor results are essentially unaffected by basis set. The B3LYP results are in significantly better agreement with experiment. As for the HF results, the B3LYP predictions are $\sim$ independent of the basis set size. However, the B3LYP dissociation energy is still smaller than the experimental value by $0.28 \mathrm{eV}$. The bond distance is too large and the vibrational frequency is too small.

As one might suspect, the MP2 and $\operatorname{CCSD}(T)$ values are much more sensitive to the basis set. The methods that include electron correlation require large basis sets, since electrons, trying to avoid each other, need more degrees of freedom in Hilbert space. The MP2 values obtained with the small basis set are in better agreement with experiment than those obtained using the large basis set. This is an example of the cancellation of basis set error with the error introduced due to the incomplete treatment of electron correlation. However, the MP2 values are much closer to the experimental ones than are the values obtained using B3LYP. Even using the small basis set, the CCSD(T) results are in very good agreement with experiment, and they improve with the large basis set. The $\operatorname{CCSD}(\mathrm{T})$ values in Table 1 are in good agreement with experiment ${ }^{28}$ and with the results of Hess and Kaldor calculated with the basis set counterpoise-corrected relativistic $\operatorname{CCSD}(\mathrm{T})$ method and a very large basis set ${ }^{29}$. Plane-wave DFT ( $\mathrm{PW}^{30}$ and $\mathrm{PBE}^{31}$ functionals) gives reasonable results.

Binding energies of molecular hydrogen. The binding energies of the first and second hydrogen molecules to the neutral gold clusters, summarized in Tables 2 and 3, were determined from

$$
\begin{gathered}
E_{b l}=E\left[A u_{n}\right]+E\left[H_{2}\right]-E\left[A u_{n} H_{2}\right] \\
E_{b 2}=E\left[A u_{n} H_{2}\right]+E\left[H_{2}\right]-E\left[A u_{n}\left(H_{2}\right)_{2}\right] .
\end{gathered}
$$


Here $n=2,3$ is the number of atoms in the specific gold cluster and $E[X]$ is the energy of the molecule $X$. The binding energies of the hydrogen molecules to the negatively charged gold clusters were determined in an analogous manner. If $\mathrm{H}_{2}$ does not bind to a gold cluster as molecular hydrogen, it can still bind as two separate hydrogen atoms, as long as the reactants have sufficient energy to break the $\mathrm{H}-\mathrm{H}$ bond.

All levels of theory predict that the first $\mathrm{H}_{2}$ binds to neutral $\mathrm{Au}_{2}$ (Table 2). Using the large basis set, the MP2 $(0.69 \mathrm{eV})$ and B3LYP $(0.42 \mathrm{eV})$ binding energies bracket the $\operatorname{CCSD}(\mathrm{T})$ result of $0.55 \mathrm{eV}$. The plane wave (PW)-PW91 and PW-rPBE methods $(0.59 \mathrm{eV}$ and $0.42 \mathrm{eV}$ respectively) are in good agreement with $\operatorname{CCSD}(\mathrm{T})$. The second $\mathrm{H}_{2}$ binds to $\mathrm{Au}_{2} \mathrm{H}_{2}$ with a smaller bonding energy than that of the first $\mathrm{H}_{2}$ (cf., Table 2). PW-PW91 predicts a very small $(0.02 \mathrm{eV})$ binding energy for the first $\mathrm{H}_{2}+\mathrm{Au}_{2}{ }^{-}$. All other methods find that molecular hydrogen does not bind to the negatively charged $\mathrm{Au}_{2}{ }^{-}$cluster.

To estimate the error introduced by using the small basis set for geometry optimizations, the geometries of $\mathrm{Au}_{2} \mathrm{H}_{2}$ were re-optimized using the large basis set. The difference in large basis set binding energies obtained using the small vs. large basis sets geometries is only $0.02 \mathrm{eV}$. Geometry optimizations for all other complexes were therefore carried out using the small basis set. However, note that although the basis set effects on predicted geometries are small, there are significant basis set effects on the predicted binding energies. For all methods, the binding energies increase when the large basis set is employed.

The equilibrium geometries of the gold clusters with adsorbed hydrogen molecules are shown in Fig. 1. All complexes are planar. The two $\mathrm{H}_{2}$ hydrogen atoms coordinate to the same gold atom, in a symmetric $\left(\mathrm{C}_{2 \mathrm{v}}\right)$ manner. The lengths of the $\mathrm{H}-\mathrm{H}$ bonds increase by 0.05-0.10 $\AA$, compared with free hydrogen molecule $(0.734 \AA)$. Addition of the first $\mathrm{H}_{2}$ stretches the Au-Au bond length by $\sim 0.04 \AA$. The effect of the second $\mathrm{H}_{2}$ is much smaller, consistent with the smaller binding energy.

The binding energies of the first and second hydrogen molecules to the $\mathrm{Au}_{3}$ clusters are given in Table 3. Again, the negatively charged cluster does not bind molecular hydrogen. The binding energy of the first $\mathrm{H}_{2}$ to the neutral $\mathrm{Au}_{3}$ cluster according to 
$\operatorname{CCSD}(\mathrm{T}) /$ large is $0.71 \mathrm{eV}$. It was not possible to carry out the $\operatorname{CCSD}(\mathrm{T})$ calculation of the binding energy of the second $\mathrm{H}_{2}$ to the $\mathrm{Au}_{3}$ cluster with the large basis set because of the large number (nearly 400 ) of basis functions. However, the $\operatorname{CCSD}(T) /$ large results can be estimated from the CCSD(T)/small calculations and MP2 calculations with both basis sets, in analogy with the procedures used in the GN methods ${ }^{32}$. Assuming that $\mathrm{E}[\mathrm{CCSD}(\mathrm{T}) / \mathrm{large}]-$ $\mathrm{E}[\mathrm{CCSD}(\mathrm{T}) / \mathrm{small}]$ is the same as the corresponding MP2 energy difference, the relative $\operatorname{CCSD}(\mathrm{T}) /$ large energy at the highest transition state is estimated to be $0.08 \mathrm{eV}+0.17 \mathrm{eV}=$ $0.25 \mathrm{eV}$. The MP2 and B3LYP and PW-rPBE binding energies are in good and PW-PW91 values are in perfect agreement with the $\operatorname{CCSD}(\mathrm{T})$ values.

Dissociation of molecular hydrogen on gold clusters. The reaction path for dissociation of molecular hydrogen on the neutral $\mathrm{Au}_{2}$ cluster is presented in Fig. 2. The energies of the transition state and reaction products are shown relative to the energy of the separated $\mathrm{Au}_{2}$ and $\mathrm{H}_{2}$. The values obtained from calculations using the large basis set are shown in parentheses. The relative energies of the transition state and reaction products obtained with most methods are in good agreement with each other. However, the PW-PW91 calculations under-estimate the barrier by $0.21 \mathrm{eV}$ and over-estimate the stability of the product by $0.14 \mathrm{eV}$. Still, this should be considered reasonable accuracy if the calculations are used for answering qualitative questions. The PW-rPBE method gives much better results than PW-PW91 for the barrier and stability of the product. The PW-rPBE energy of the product is in perfect agreement with $\operatorname{CCSD}(\mathrm{T})$ and the barrier is underestimated by only 0.08 $\mathrm{eV}$. Of course, this method has been parametrized for system like the one under study; it is not clear how well the success seen here will carry over to other systems. The CCSD(T) activation energy for the dissociation of molecular hydrogen on the $\mathrm{Au}_{2}$ cluster is $1.10 \mathrm{eV}$ ( $0.55 \mathrm{eV}$ above separated reactants). The $\mathrm{Au}_{2}$ cluster with one bridging and one terminal hydrogen atom is $0.22 \mathrm{eV}$ higher in energy than $\mathrm{Au}_{2}$ with an adsorbed $\mathrm{H}_{2}$ molecule, so the dissociation is predicted to be mildly endothermic.

All methods make the same qualitative predictions: $\mathrm{H}_{2}$ binds to $\mathrm{Au}_{2}$ and has a fairly high barrier to dissociation. The state with the dissociated hydrogen is metastable with respect to the adsorbed molecular hydrogen. The barriers are such that the adsorbed $\mathrm{H}_{2}$ would much rather desorb than dissociate. 
As noted above, while there is no stable $\mathrm{Au}_{2}{ }^{-} \mathrm{H}_{2}$ cluster, there is a stable $\mathrm{Au}_{2} \mathrm{H}_{2}^{-}$ species in which the molecular hydrogen has dissociated. This is illustrated in Figure 3. $\operatorname{CCSD}(\mathrm{T}) /$ /arge predicts the largest barrier for this reaction $(0.93 \mathrm{eV}$ relative to separated $\mathrm{Au}_{2}{ }^{-}$and $\mathrm{H}_{2}$ ). The transition state has an asymmetrically bound $\mathrm{H}_{2}$ molecule with a significantly stretched (1.085 $\AA$ ) H-H bond, compared with free hydrogen molecule $(0.734$ $\AA)$. There is one very shallow minimum and one very low energy transition state on this reaction path. The transition state is only $0.01 \mathrm{eV}$ higher in energy than the minimum and therefore may not exist. The presence of these stationary points illustrates that even such a seemingly simple reaction can have a nontrivial potential energy surface. However, these local minima and transition state probably do not play a significant role in the mechanism of $\mathrm{H}_{2}$ dissociation. The PW-PW91 and PW-rPBE DFT methods predict the existence of a shoulder on this reaction path instead of a very shallow minimum and transition state with very small activation energy. Interestingly, for this reaction PW-rPBE gives a barrier that is in better agreement with the CCSD(T) result than the PW-PW91 barrier. However, the PWPW91 energy of the product relative to reactants is closer to the CCSD(T) value than is the PW - PBE product energy. Both functionals under-estimate the barrier and stability of the product.

The dissociation of $\mathrm{H}_{2}$ on the $\mathrm{Au}_{3}$ cluster (Fig. 4) is more complex and characterized by several minima and transition states. Because of the large number of basis functions and the low symmetry of some of the stationary points, it was not possible to carry out open-shell $\operatorname{CCSD}(\mathrm{T})$ calculations with the large basis set for all of these species. However, the $\mathrm{CCSD}(\mathrm{T}) /$ large energies of $\mathrm{Au}_{3} \mathrm{H}_{2}$ can be estimated using the same procedure as was used to estimate the binding energy of the second $\mathrm{H}_{2}$ to the $\mathrm{Au}_{3}$ cluster. The dependence of the results on the size of the basis set is quite significant for this reaction. All methods except PW-PW91 are in good agreement with each other. The PW-PW91 and PW-rPBE potential energy surfaces appear to be too flat.

After bonding to the $\mathrm{Au}_{3}$ cluster, with a binding energy of $0.71 \mathrm{eV}, \mathrm{H}_{2}$ can dissociate through a symmetric transition state with an estimated $\operatorname{CCSD}(\mathrm{T})$ activation energy of $0.18 \mathrm{eV}$ However, the minimum on the potential energy surface that corresponds to two hydrogen atoms, symmetrically attached to one gold atom, is almost isoenergetic with this transition 
state. Therefore, the lifetime of a $\mathrm{Au}_{3}$ cluster with two hydrogen atoms attached to one gold atom is likely to be very small. In order to form the $\mathrm{Au}_{3} \mathrm{H}_{2}$ complex with dissociated hydrogen and a significant lifetime, the reaction must proceed further through an asymmetric transition state towards the complex with one bridging and one terminating hydrogen atom. The estimated MP2 and $\operatorname{CCSD}(\mathrm{T})$ activation energies for this reaction, with respect to the $\mathrm{Au}_{3} \mathrm{H}_{2}$ complex with molecular hydrogen, are 0.69 and $0.59 \mathrm{eV}$, respectively. It is important to point out that these values do not depend on the basis set used. For comparison PW-PW91 and PW-rPBE values are $0.30 \mathrm{eV}$ and $0.28 \mathrm{eV}$, respectively .

The mechanism of $\mathrm{H}_{2}$ dissociation on the negatively charged $\mathrm{Au}_{3}{ }^{-}$cluster is shown in Fig. 5. Molecular hydrogen does not form a stable complex with this gold anion. The $\operatorname{CCSD}(\mathrm{T})$ /large barrier for the $\mathrm{H}_{2}$ dissociation is $1.39 \mathrm{eV}$. B3LYP, MP2 and $\operatorname{CCSD}(\mathrm{T})$ give very similar values for the barrier, whereas the PW-PW91 and PW-rPBE values are again too low.

Finally, in order to explore the possibility of other bridging structures on the small gold clusters, MP2 calculations were performed to search for double bridged structures that might correspond to minima on the potential energy surfaces of $\mathrm{H}_{2}$ with neutral and negatively charged $\mathrm{Au}_{2}$ and $\mathrm{Au}_{3}$ clusters. No such local minima have been found. Similarly, for $\mathrm{Au}_{3}$ and $\mathrm{Au}_{3}{ }^{-}$no stable complexes with $\mathrm{H}$ atom coordinated to all three gold atoms were found.

\section{Summary}

It has been shown that one and even two $\mathrm{H}_{2}$ molecules easily bind to neutral $\mathrm{Au}_{2}$ and $\mathrm{Au}_{3}$ clusters. According to the best $\mathrm{CCSD}(\mathrm{T})$ calculations, the activation energies for dissociation of $\mathrm{H}_{2}$ adsorbed on neutral $\mathrm{Au}_{2}$, and $\mathrm{Au}_{3}$ clusters are $1.10 \mathrm{eV}$ and $0.59 \mathrm{eV}$. On the other hand, the activation energies with respect to separate Au clusters and $\mathrm{H}_{2}$ molecules are only $0.55 \mathrm{eV}$ and $-0.12 \mathrm{eV}$ (transition state is lower in energy than separated $\mathrm{Au}_{3}$ and $\mathrm{H}_{2}$ ). This means that some $\mathrm{H}_{2}$ molecules can dissociate on these clusters directly without formation of weakly bound $\mathrm{H}_{2}$ - gold cluster complexes. But since these weak complexes 
correspond to global minima on the potential energy surfaces, their population at equilibrium should be significant.

Molecular hydrogen does not form stable complexes with negatively charged $\mathrm{Au}_{2}{ }^{-}$ and $\mathrm{Au}_{3}{ }^{-}$clusters. The dissociation barriers for $\mathrm{H}_{2}$ on these clusters (with respect to the separate cluster and $\left.\mathrm{H}_{2}\right)$ are $0.93 \mathrm{eV}\left(\mathrm{Au}_{2}{ }^{-}\right)$and $1.39 \mathrm{eV}\left(\mathrm{Au}_{3}{ }^{-}\right)$. Since the energies of $\mathrm{Au}_{2}{ }^{-}$ and $\mathrm{Au}_{3}{ }^{-}$with adsorbed dissociated $\mathrm{H}_{2}$ are $0.49 \mathrm{eV}$ and $0.96 \mathrm{eV}$, respectively, lower than the energies of separated $\mathrm{H}_{2}$ and gold clusters, formation of these adsorbed complexes is possible if the reactants have sufficient energy to overcome the barriers and if collisions with an inert gas dissipate the adsorption energy.

It is interesting to compare the adsorption characteristics of $\mathrm{H}_{2}$ vs. $\mathrm{O}_{2}$ to small gold clusters. In contrast to oxygen adsorption ${ }^{7}$ the DFT methods perform reasonably well for predicting geometries and hydrogen adsorption on small gold clusters, at least with regard to the prediction of binding energies. The plane wave basis set with the PW91 and rPBE functionals, predicts binding energies that are very similar to the $\operatorname{CSSD}(\mathrm{T})$ values with the large Gaussian basis set. However, the PW-PW91 and PW-rPBE method tends to underestimate activation energies.

The small basis set used here appears to be sufficient for optimization of the geometries and calculations of the relative energies of minima and transition states. For quantitative prediction of binding energies of $\mathrm{H}_{2}$ to gold clusters it is necessary to use the large basis set, as there are significant basis set effects. The small basis set appears to be sufficient for predicting the values of $\mathrm{O}_{2}$ binding energies. ${ }^{33}$ The difference may be associated with the small number of Gaussian functions on hydrogen atom in the 6-31G(d) basis set.

The molecular hydrogen adsorption on small gold clusters is very different from that of molecular oxygen. ${ }^{7} \mathrm{H}_{2}$ coordinates to gold clusters with both hydrogen atoms, whereas, only one oxygen atom of $\mathrm{O}_{2}$ coordinates to a gold cluster. Molecular hydrogen does not bind to negatively charged $\mathrm{Au}_{\mathrm{n}}{ }^{-}$clusters. In contrast, oxygen binding mostly depends on the presence of unpaired electrons on the gold clusters, and is associated with partial electron transfer from the gold cluster to $\mathrm{O}_{2}$. Of the clusters studied in this paper, only neutral $\mathrm{Au}_{3}$ can bind individual $\mathrm{H}_{2}$ and $\mathrm{O}_{2}$ molecules, with $\mathrm{O}_{2}$ and $\mathrm{H}_{2}$ binding energies of $0.17 \mathrm{eV}$ and 0.71 
eV respectively. $\mathrm{H}_{2}$ binds to neutral $\mathrm{Au}_{2}$, while $\mathrm{O}_{2}$ does not. On the other hand, $\mathrm{O}_{2}$ binds to $\mathrm{Au}_{2}{ }^{-}$, while $\mathrm{H}_{2}$ does not. Therefore, one can speculate that simultaneous adsorptions of $\mathrm{O}_{2}$ and $\mathrm{H}_{2}$ are possible only on neutral clusters with odd number of gold atoms. Exploring the validity of this hypothesis and its relevance to catalytic properties of small gold clusters will be the focus of future work.

\section{Acknowledgments}

The authors thank Dr. Michael Schmidt and Dr. Dmitri Fedorov for helpful discussions. This work was supported by AFOSR through a DURINT grant. The calculations in this work were performed in part on an IBM workstation cluster made possible by grants from IBM in the form of a Shared University Research grant and the United States Department of Energy.

\section{References}

${ }^{1}$ R.J. Bartlett, J. Phys. Chem., 93, 1697 (1989); C. Hampel, K. Peterson, and H.-J. Werner, Chem. Phys. Lett. 190, I (1992) and references therein. The program to compute the perturbative triples corrections has been developed by M.J.O. Deegan and P.J. Knowles (1992).

${ }^{2}$ A. Sarkany and Z. Revay, Applied Catalysis a-General 243, 347 (2003).

${ }^{3} \mathrm{C}$. Mohr, H. Hofmeister, and P. Claus, Journal of Catalysis 213, 86 (2003); C. Mohr, H. Hofmeister, J. Radnik, and P. Claus, J.Am.Chem.Soc. 125, 1905 (2003).

${ }^{4}$ J. Jia, K. Haraki, J. N. Kondo, K. Domen, and K. Tamaru, J. Phys. Chem. B 104, 11153 (2000); A. Sarkany and Z. Revay, Applied Catalysis a-General 243, 347 (2003); T. V. Choudhary, C. Sivadinarayana, A. K. Datye, D. Kumar, and D. W. Goodman, Catalysis Letters 86, 1 (2003).

${ }^{5}$ G. C. Bond and D. T. Thompson, Catalysis by gold, Catal. Rev. - Sci. Eng. 41, 319 (1999); G. J. Hutchins, Cat. Today 72, 11-17 (2002); G. C. Boyd, Cat. Today 72, 5 (2002); M. Haruta, Cat. Today 36, 153 (1997); H. Haruta and M. Date, Appl. Cat. A 222, 427 (2001). 
${ }^{6}$ B. Hammer and J. K. Norskov, in Advances in Catalysis, Vol 45 (2000), pp. 71-129. ; P. Kratzer and M. Scheffler, Computing in Science \& Engineering 3, 16 (2001); J. K. Norskov, in Reaction Kinetics and the Development of Catalytic Processes (1999), pp. 3-10 ; J. K. Norskov, Nature 414, 405 (2001).

${ }^{7}$ S.A. Varganov, R.M. Olson, M.S. Gordon, H. Metiu, J. Chem. Phys. 119, 2531 (2003).

${ }^{8}$ E. Wahlstrom, N. Lopez, R. Schaub, P. Thostrup, A. Ronnau, C. Africh, E. Laegsgaard, J. K. Norskov, and F. Besenbacher, Phys. Rev. Lett. 90 (2003); R. Schaub, E. Wahlstrom, A. Ronnau, E. Laegsgaard, I. Stensgaard, and F. Besenbacher, Science 299, 377 (2003).

${ }^{9}$ A. Vijay, G. Mills, and H. Metiu, J. Chem. Phys. 118, 6536-6551 (2003); U. Diebold, Surface Sci. Rep. 48, 53 (2003).

${ }^{10}$ R. Schaub, R. Thostrup, N. Lopez, E. Laegsgaard, I. Stensgaard, J. K. Norskov, and F. Besenbacher, Phys. Rev. Lett. 87, 266104 (2001); U. Diebold, Surface Sci. Rep. 48, 53 (2003).

${ }^{11}$ A. Sanchez, S. Abbet, U. Heiz, W. D. Schneider, H. Hakkinen, R. N. Barnett, and U. Landman, J.Phys.Chem. A 103, 9573 (1999).

${ }^{12}$ C. Møller, M.S. Plesset, Phys. Rev. 46, 618 (1934).

${ }^{13}$ K.Ishida, K.Morokuma, A.Komornicki, J.Chem.Phys. 66, 2153 (1977).

${ }^{14}$ C.Gonzales, H.B.Schlegel, J. Chem. Phys. 90, 2154 (1989).

${ }^{15}$ T.J. Lee, D. Jayatilaka, Chem. Phys. Lett. 201, 1 (1993); T.J. Lee, A.P. Rendell, K.G. Dyall, D. Jayatilaka, J. Chem. Phys. 100, 7400 (1994).

${ }^{16}$ P.J. Knowles, C. Hampel, and H.-J. Werner, J. Chem. Phys. 99, 5219 (1993).

${ }^{17}$ A.D. Becke, J. Chem. Phys. 98, 5648 (1993); P.J. Stephens, F.J. Devlin, C.F. Chablowski, M.J. Frisch, J. Phys. Chem. 98, 11623 (1994); R.H. Hertwig, W. Koch, Chem. Phys. Lett. 268,345 (1997).

${ }^{18}$ W.J. Stevens, M. Krauss, H. Basch, P.G. Jasien, Can. J. Chem. 70, 612 (1992).

${ }^{19}$ W.J. Hehre, R. Ditchfield and J.A. Pople, J. Chem. Phys. 56, 2257 (1972).

${ }^{20}$ T.H. Dunning, Jr. J. Chem. Phys, 90, 1007 (1989). Basis sets were obtained from the Extensible Computational Chemistry Environment Basis Set Database, Version 6/19/03, as developed and distributed by the Molecular Science Computing Facility, Environmental and Molecular Sciences Laboratory which is part of the Pacific Northwest Laboratory, P.O. Box 
999, Richland, Washington 99352, USA, and funded by the U.S. Department of Energy. The Pacific Northwest Laboratory is a multi-program laboratory operated by Battelle Memorial Institute for the U.S. Department of Energy under contract DE-AC06-76RLO 1830.

${ }^{21}$ Y. Wang, J.P. Perdew, Phys. Rev. B 44, 13298 (1991).

${ }^{22}$ B. Hammer, L. B. Hansen, and J. K. Norskov, Phys. Rev. B, 59, 7413 (1999).

${ }^{23} \mathrm{G}$. Kresse and J. Joubert, Phys. Rev. B, 59, 1758 (1999).

${ }^{24}$ G. Kresse and J. Hafner, Phys. Rev. B, 47, 558 (1993); G. Kresse and J. Hafner, Phys. Rev. B, 49, 14251 (1994); G. Kresse and J. Furthmuller, Comput. Mater. Sci., 6, 15 (1996); G. Kresse and J. Furthmuller, Phys. Rev. B, 54, 11169 (1996).

${ }^{25}$ G. Makov and M. C. Payne, Phys. Rev. B, 51, 4014 (1995).

${ }^{26}$ M.W. Schmidt, K.K. Baldridge, J. A. Boatz, S.T. Elbert, M.S. Gordon, J.H. Jensen, S. Koseki, N. Matsunaga, K.A. Nguyen, S.J. Su, T.L. Windus, M. Dupuis, J.A. Montgomery, J. Comp.Chem. 14, 1347 (1993); G.D. Fletcher, M.W. Schmidt, M.S. Gordon, Adv. Chem. Phys. 110, 267 (1999); G.D. Fletcher, M.W. Schmidt, B.M. Bode, M.S. Gordon, Comput. Phys. Commun. 128, 190 (2000).

${ }^{27}$ MOLPRO is a package of ab-initio programs written by H.-J. Werner and P.J. Knowles, with contributions from R.D. Amos, A. Bernhardsson, A. Berning, P. Celani, D.L. Cooper, M.J.O. Deegan, A.J. Dobbyn, F. Eckert, C. Hampel, G. Hetzer, T. Korona, R. Lindh, A.W. Lloyd, S.J. McNicholas, F.R. Mandy, W. Meyer, M.E. Mura, A. Nicklass, P. Palmieri, R. Pitzer, G. Rauhut, M. Schutz, H. Stoll, A.J. Stone, R. Tarroni, and T. Thorsteinsson.

${ }^{28}$ K.P. Huber, G. Herzberg, Molecular Spectra and Molecular Structure. IV. Constants of Diatomic Molecules, Van Nostrand, New York, 1979.

${ }^{29}$ B.A. Hess, U. Kaldor, J. Chem. Phys. 112, 1809 (2000).

${ }^{30}$ G. Mills, M.S. Gordon, H. Metiu, Chem. Phys. Lett. 359, 493 (2002).

${ }^{31}$ H. Hakkinen and U. Landman, J. Am. Chem. Soc. 123, 9704 (2001).

${ }^{32}$ L.A. Curtiss, K. Raghavachari, P.C. Redfern, V. Rassolov, J.A. Pople, J. Chem. Phys. 109, 7764 (1998).

${ }^{33}$ S.A. Varganov, R,M. Olson, M.S. Gordon, H. Metiu (unpublished). 
Table 1. Bond distances $R(\AA)$, dissociation energies $D_{e}(e V)$ and vibration frequencies $\omega_{c}(1 / \mathrm{cm})$ of the $\mathrm{Au}_{2}$ at different level of theory.

\begin{tabular}{lcccccc}
\hline & \multicolumn{3}{c}{ Small basis set } & \multicolumn{3}{c}{ Large basis set } \\
\hline & $\mathrm{R}(\AA)$ & $\mathrm{D}_{e}(\mathrm{eV})$ & $\omega_{e}(1 / \mathrm{cm})$ & $\mathrm{R}(\AA)$ & $\mathrm{D}_{\mathrm{e}}(\mathrm{eV})$ & $\omega_{\mathrm{e}}(1 / \mathrm{cm})$ \\
\hline $\mathrm{HF}$ & 2.615 & 0.80 & 156 & 2.598 & 0.88 & 156 \\
MP2 & 2.494 & 2.24 & 190 & 2.448 & 2.47 & 200 \\
CCSD(T) & 2.535 & 2.04 & 175 & 2.504 & 2.14 & \\
B3LYP & 2.562 & 1.98 & 167 & 2.539 & 2.03 & 168 \\
\hline PW-PBE $^{\mathrm{a}}$ & & & & 2.54 & 2.22 & \\
PW-PW91 $^{b}$ & & & & 2.528 & 2.27 & \\
${\text { Cp-CCSD }(T)^{\mathrm{c}}}^{\mathrm{c}}$ & & & & 2.488 & 2.19 & 187 \\
Experiment $^{\mathrm{d}}$ & & & & 2.472 & 2.29 & 191 \\
\hline
\end{tabular}

DFT with plane-wave basis set and PBE functional (Ref. 32).

${ }^{b}$ DFT with plane-wave basis set and PW91 functional (Ref. 31).

${ }^{\mathrm{C}}$ Conterpose-corrected CCSD(T) with all electron PJHN-4f2g1h1i basis set (Ref. 29).

${ }^{\mathrm{d}}$ Experimental values (Ref. 28). 
Table 2. Small (large) basis $\mathrm{Au}_{2}^{-}$and $\mathrm{Au}_{2}$ binding energies (eV) for the first, $\mathrm{E}_{\mathrm{bl}}$, and the second, $\mathrm{E}_{\mathrm{b} 2}, \mathrm{H}_{2}$ molecule.

\begin{tabular}{lccccc}
\hline & MP2 & CCSD(T) & B3LYP & PW-PW91 & PW-rPBE \\
\hline $\mathrm{Au}_{2}, \mathrm{E}_{\mathrm{b} 1}$ & $0.39(0.69)$ & $0.27(0.55)$ & $0.30(0.42)$ & 0.59 & 0.42 \\
$\mathrm{Au}_{2}, \mathrm{E}_{\mathrm{b} 2}$ & $0.19(0.37)$ & $0.12(0.29)$ & $0.15(0.24)$ & 0.37 & 0.19 \\
$\mathrm{Au}_{2}{ }^{-}, \mathrm{E}_{\mathrm{b} 1}$ & unstable & - & unstable & 0.02 & unstable \\
$\mathrm{Au}_{2}^{-}, \mathrm{E}_{\mathrm{b} 2}$ & unstable & - & unstable & unstable & unstable \\
\hline
\end{tabular}


Table 3. Small (large) basis $\mathrm{Au}_{3} / \mathrm{Au}_{3}{ }^{-}$Binding energies (eV) for the first, $\mathrm{E}_{\mathrm{b} 1}$, and the second, $\mathrm{E}_{\mathrm{b} 2}, \mathrm{H}_{2}$ molecule.

\begin{tabular}{lccccc}
\hline & MP2 & CCSD(T) & B3LYP & PW-PW91 & PW-rPBE \\
\hline $\mathrm{Au}_{3}, \mathrm{E}_{\mathrm{b} 1}$ & $0.58(0.90)$ & $0.41(0.71)$ & $0.39(0.52)$ & 0.71 & 0.53 \\
$\mathrm{Au}_{3}, \mathrm{E}_{\mathrm{b} 2}$ & $0.17(0.34)$ & $0.08\left(0.25^{\mathrm{a}}\right)$ & $0.11(0.18)$ & 0.26 & 0.08 \\
$\mathrm{Au}_{3}{ }^{-}, \mathrm{E}_{\mathrm{b} 1}$ & unstable & - & unstable & 0.05 & unstable \\
$\mathrm{Au}_{3}{ }^{-}, \mathrm{E}_{\mathrm{b} 2}$ & unstable & - & unstable & unstable & unstable \\
\hline
\end{tabular}

${ }^{a} \operatorname{CCSD}(\mathrm{T}) /$ large values was estimated based on $\mathrm{CCSD}(\mathrm{T}) / \mathrm{small}$, MP2/small and MP2/large values (see explanation in the text). 


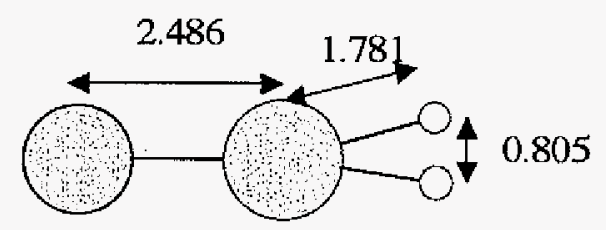

$\mathrm{Au}_{2} \mathrm{H}_{2}{ }^{1} \mathrm{~A}_{1}$

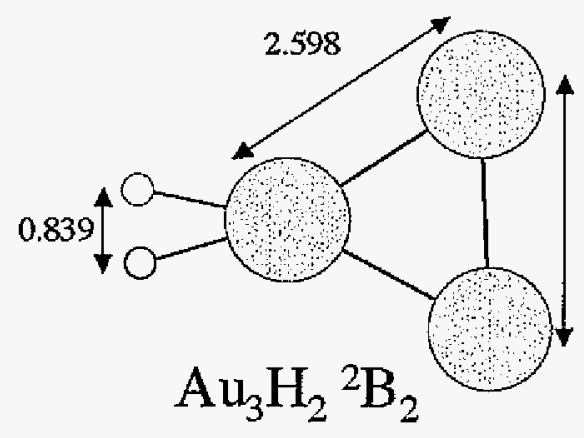

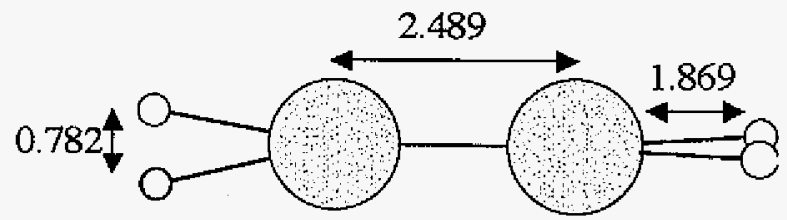

$\mathrm{Au}_{2} \mathrm{H}_{4}{ }^{1} \mathrm{~A}_{1}$

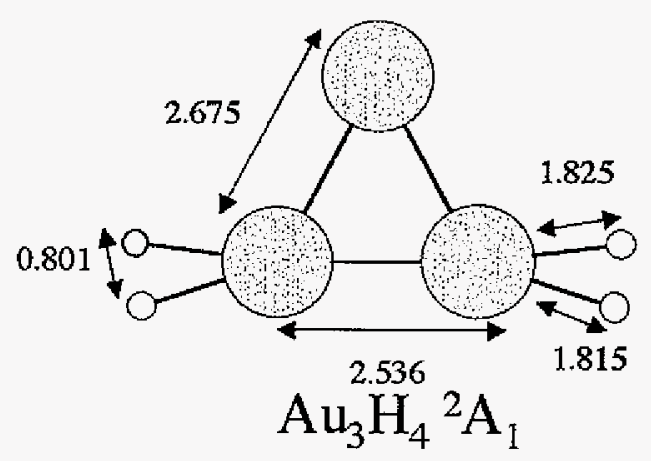

Figure 1. MP2/large optimized geometries for lowest energy ground states of $\mathrm{Au}_{2} \mathrm{H}_{2}, \mathrm{Au}_{2} \mathrm{H}_{4}$, $\mathrm{Au}_{2} \mathrm{H}_{2}^{-}$and $\mathrm{Au}_{2} \mathrm{H}_{4}^{-}$complexes. Bond distances are in angstroms. 
61

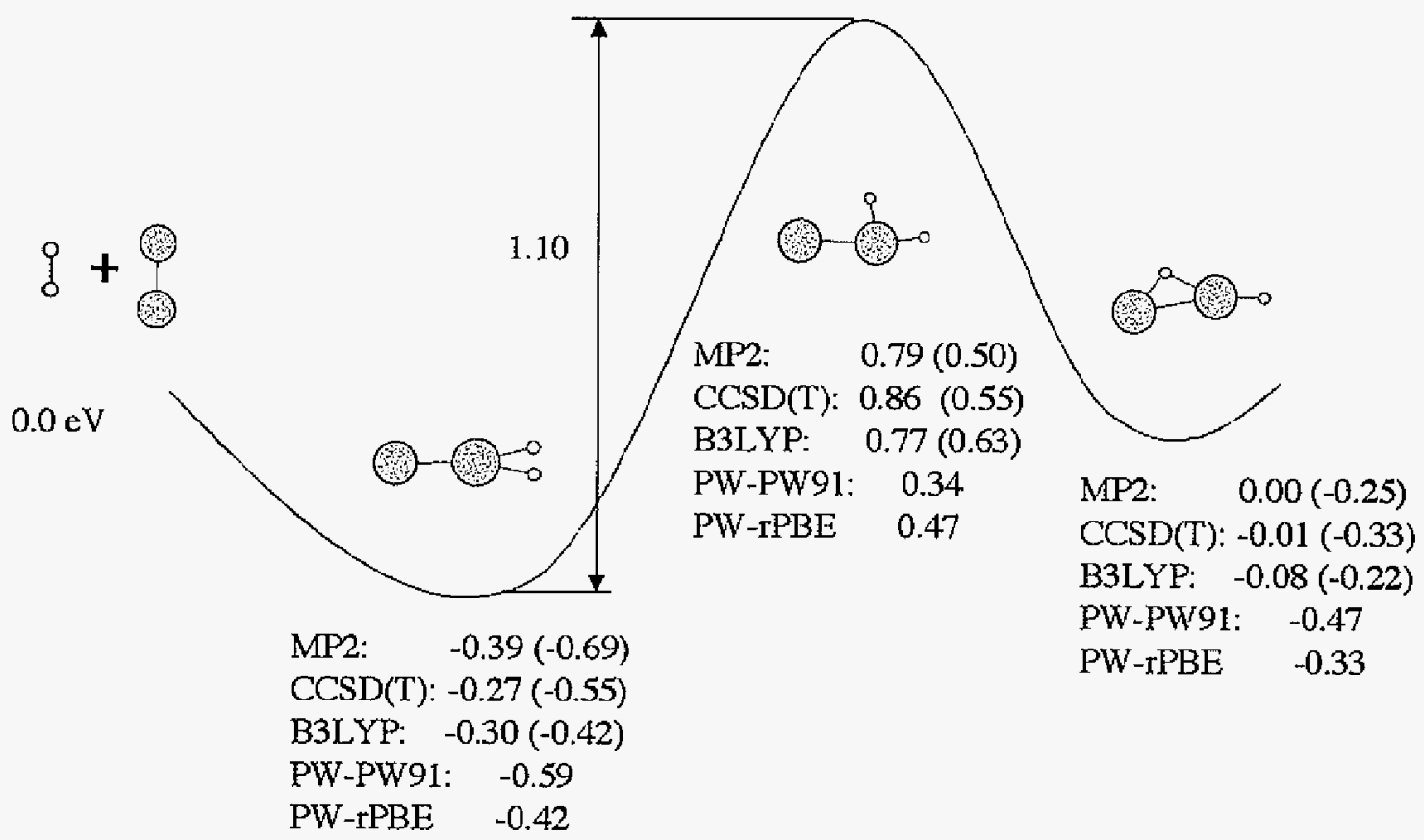

Figure 2. Reaction path for $\mathrm{H}_{2}$ dissociation on neutral $\mathrm{Au}_{2}$, with relative energies of minima and transition state. The energies calculated with the large basis set are in parentheses. 


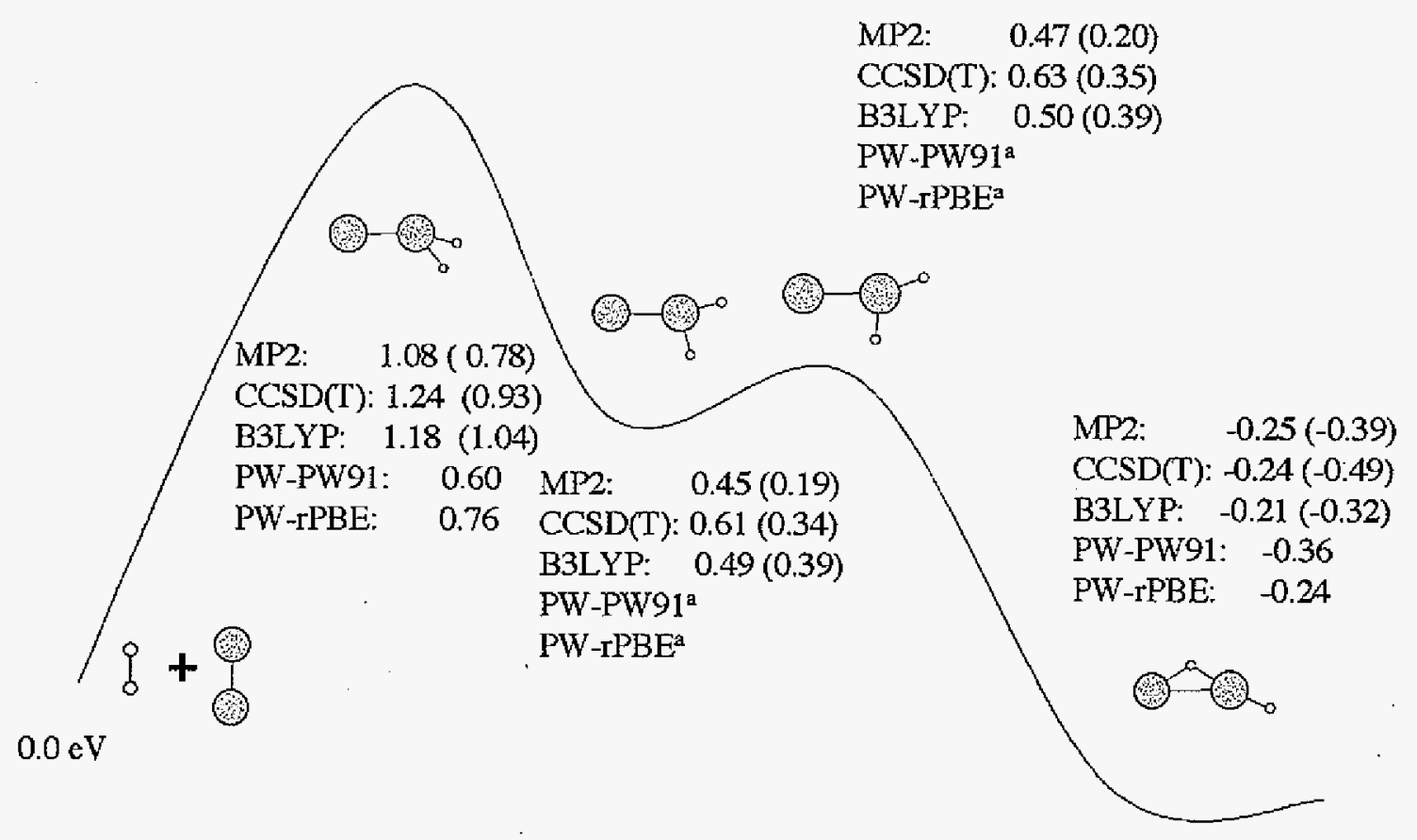

Figure 3. Reaction path for $\mathrm{H}_{2}$ dissociation on negatively charged $\mathrm{Au}_{2}{ }^{-}$, with relative energies of minima and transition states. The energies calculated with the large basis set are in parentheses. ${ }^{\text {T}}$ There is a shoulder instead of a minimum and transition state on the PW-PW91 and PW-rPBE reaction paths. 


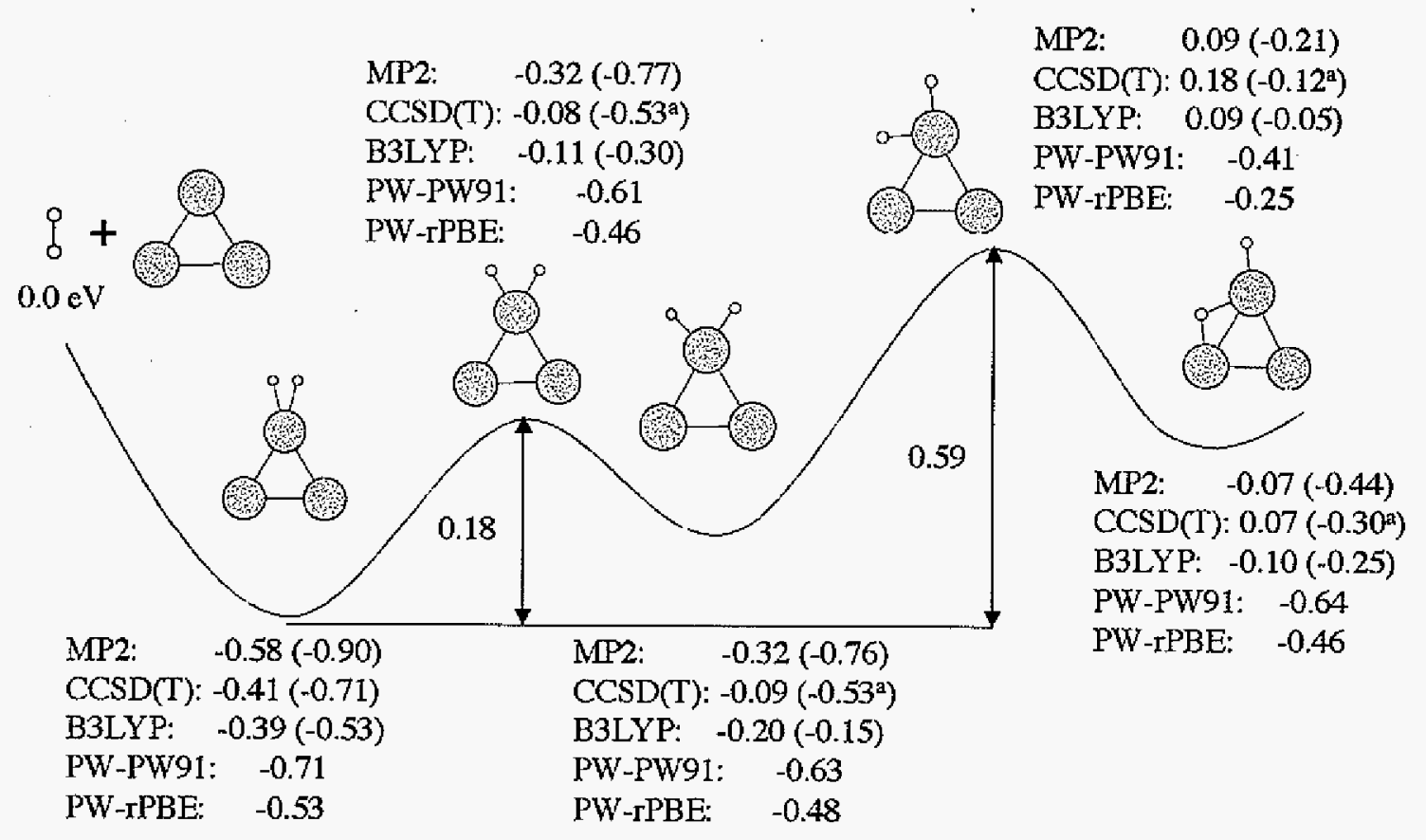

Figure 4. Reaction path for $\mathrm{H}_{2}$ dissociation on neutral $\mathrm{Au}_{3}$, with relative energies of minima and transition states. The energies calculated with the large basis set are in parentheses. $\operatorname{CCSD}(\mathrm{T}) /$ large values were estimated based on $\operatorname{CCSD}(\mathrm{T}) /$ small, MP2/small and MP2/large values (see explanation in the text). 


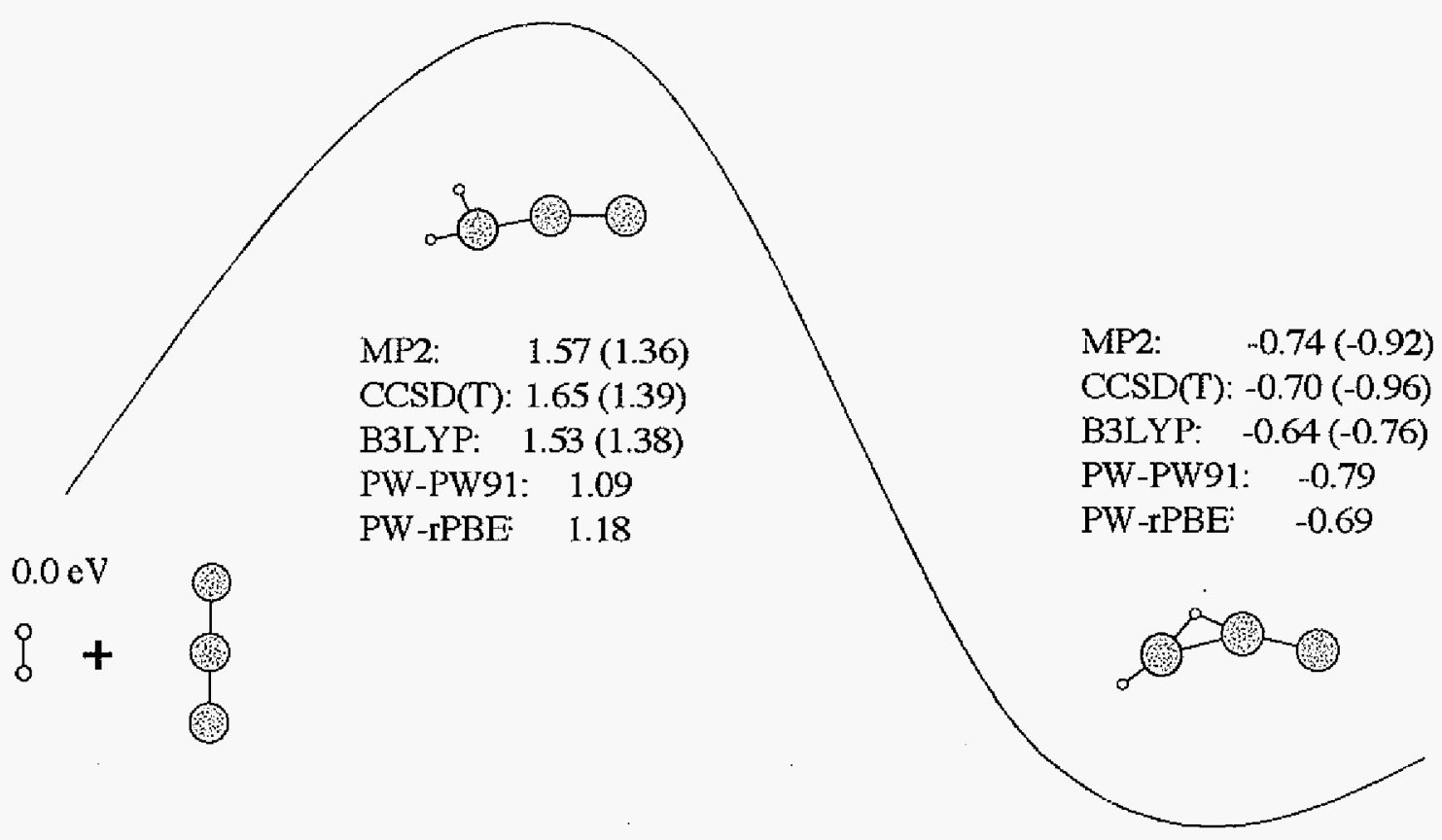

Figure 5. Reaction path for $\mathrm{H}_{2}$ dissociation on neutral $\mathrm{Au}_{3}{ }^{-}$, with relative energies of minima and transition states. The energies calculated with the large basis set are in parentheses. 


\title{
CHAPTER 4. A STUDY OF THE ISOMERS OF $C_{36}$ FULLERENE USING SINGLE AND MULTIREFERENCE MP2 PERTURBATION THEORY
}

Taken from a paper that has been published in the Chemical Physics Letters 362,380 , (2002).

Copyright 2002 Elsevier Science.

Sergey A. Varganov, Paul V. Avramov, Sergey. G. Ovchinnikov and Mark S. Gordon

\begin{abstract}
The role of electron correlation in the five lowest energy isomers of $C_{36}$ fullerene is studied using the CASSCF method with single and multireference MP2 theories. It is shown that the distortion of the $\mathrm{D}_{6 \mathrm{~h}}$ isomer at HF levels of theory is an artificial effect caused by the oversimplified form of the wave function. The singlet state of the $\mathrm{D}_{6 \mathrm{~h}}$ isomer has diradical character, and it is the lowest energy state. The triplet states of the $D_{6 h}$ and $D_{2 d}$ isomers are 34 $\mathrm{kJ} / \mathrm{mol}$ and $92 \mathrm{~kJ} / \mathrm{mol}$ and the singlet state of the $\mathrm{D}_{2 \mathrm{~d}}$ isomer is $148 \mathrm{~kJ} / \mathrm{mol}$ higher in energy than the $\mathrm{D}_{6 \mathrm{~h}}$ singlet state.
\end{abstract}

\section{Introduction}

After the synthesis of a solid form of $\mathrm{C}_{36}$ fullerene by the arc-discharge method had been reported, there were a number of publications about the relative stability of different isomers of this fullerene. The comparison of experimental and theoretical NMR spectra suggests that the synthesized form of $\mathrm{C}_{36}$ consists of $\mathrm{D}_{6 \mathrm{~h}}$ isomers [1].

There are fifteen conventional fullerene isomers (cages containing five- and sixmembered rings only) of the $C_{36}$ cluster [2]. Two of them, the $D_{6 h}$ and $D_{2 d}$ isomers, have a minimal number of adjusted pentagons; this may be considered to be an indication of stability. However, it has been shown that at least one quasi-fullerene, which contains one four-member ring, has a total energy that is close to the energies of conventional isomers [3]. 
At this time it is still not clear why the $\mathrm{D}_{6 \mathrm{~h}}$ isomer is the only one observed in the experiments. According to density functional theory (DFT) and hybrid Hartree-Fock/density functional theory (HF/DFT) the $\mathrm{D}_{2 \mathrm{~d}}$ and $\mathrm{D}_{6 \mathrm{~h}}$ singlet states and $\mathrm{D}_{6 \mathrm{~h}}$ triplet state are almost isoenergetic, with the exact order of the states depending on the type of functional used [3-9]. At least two explanations of this phenomenon have been proposed. According to the first one, the entropy plays an important role in the synthesis of the $C_{36}$ fullerene [3,10-12]. The entropy contribution to the Gibbs free energy often can be neglected for reactions at low temperature. But since the synthesis of fullerenes takes place at high temperature, the entropy contribution can be quite significant. Slanina et al. [3], using a hybrid HF/DFT method, showed that even though the $\mathrm{D}_{2 \mathrm{~d}}$ isomer is the most stable one at $0 \mathrm{~K}$, the $\mathrm{D}_{6 \mathrm{~h}}$ isomer becomes the most stable isomer at temperatures higher than $\sim 500 \mathrm{~K}$. The other explanation is based on the exceptionally large electron correlation energy in the $D_{6 h}$ isomer $[13,14]$ predicted by a semi-empirical method. Fowler et al. [13] used configuration interaction (CI) calculations, including single and double excitations in the space of the four orbitals (HOMO-1, HOMO, LUMO and LUMO+1), to show that the electron correlation effect is significantly larger for the $\mathrm{D}_{6 \mathrm{~h}}$ isomer compared to other $\mathrm{C}_{36}$ isomers. Since DFT methods are not always able to estimate correlation energy correctly, especially in the case of systems with strong electron correlation, the authors who used DFT suggest that this isomer is almost isoenergetic with the $D_{2 \mathrm{~d}}$ isomer.

Interestingly, several authors suggest that the $\mathrm{D}_{6 \mathrm{~h}}$ isomer undergoes a second-order Jahn-Teller distortion which reduces the symmetry of this isomer from $D_{6 h}$ to $C_{6 v}$ or even to $\mathrm{C}_{2 \mathrm{~h}}$ symmetry $[3,13]$. Others argued that at least according to hybrid HF/DFT, such a distortion does not occur $[7,9]$.

The purpose of this study is to determine the most thermodynamically stable isomer of the $\mathrm{C}_{36}$ fullerene, using the complete active space self-consistent-field (CASSCF) method [15] and single and multireference second-order Moller-Plesset perturbation theory (MP2 [16] and MRMP2 [17]). CASSCF is a variational method in which the wave function is represented as a linear combination of Slater determinants. This method is able to recover the nondynamic part of the electron correlation. On the other hand, the MP2 and MRMP2 theories are able to account for some fraction of the dynamic part of the correlation energy. 
The possibility of Jahn-Teller distortion in $\mathrm{C}_{36}$ isomers is explored as well. The set of five isomers that are thermodynamically most stable at the hybrid HF/DFT level of theory [3] is studied. These isomers are shown in Fig. 1. The relative energies of the singlet and triplet states of these five isomers are presented. The results of calculations performed at different levels of theory are compared, and the role of the electronic correlation is discussed.

\section{Computational details}

For each electronic state of each isomer the following procedure was used. First, full geometry optimization was carried out at the semi-empirical PM3 [18] level of theory without any symmetry restriction. Second, the symmetry point group was determined and a numerical hessian was calculated and diagonalized in order to ensure that the geometry corresponds to a minimum on the potential energy surface. The PM3 optimized geometry was used as an initial guess for a HF geometry optimization in the corresponding symmetry group. Finally, the HF optimized geometry was used as a starting point for the hybrid HF/DFT geometry optimization and for MP2, CASSCF and MRMP2 single point calculations.

A restricted open shell wave function was used to describe the HF and HF/DFT triplet states. In the hybrid HF/DFT calculations a B3LYP functional which combines five functionals, namely Becke + Slater + HF exchange and LYP + VWN5 correlation [19-21] was used. In the MP2 open shell calculations the ZAPT formalism was used [22,23]. This type of open shell MP2 is based on a restricted open shell HF wave function that is free of spin contamination. In all calculations, except for the minimal basis set PM3, the 6-31G* basis set was used. Since the LUMO of the $\mathrm{D}_{2 \mathrm{~d}}$ isomer and the LUMO+1 of the $\mathrm{D}_{6 \mathrm{~h}}$ isomer are doubly degenerate, the minimal consistent CASSCF active space (Fig. 2) includes 2 electrons in 4 orbitals. This active space contains two nonbonding $\left(5 \mathrm{~b}_{1 \mathrm{u}}, 5 \mathrm{~b}_{2 \mathrm{~g}}\right)$ and a pair of two-center bonding $\left(10 \mathrm{e}_{2 \mathrm{~g}}\right)$ orbitals for the $\mathrm{D}_{6 \mathrm{~h}}$ isomer, two-center bonding $\left(12 \mathrm{~b}_{1}, 27 \mathrm{e}, 18 \mathrm{a}_{1}\right)$ orbitals for the $\mathrm{D}_{2 \mathrm{~d}}$ isomer, three two-center bonding $\left(21 \mathrm{a}_{2}, 27 \mathrm{~b}_{1}, 22 \mathrm{a}_{2}\right)$ and one three-center bonding $\left(24 \mathrm{~b}_{2}\right)$ orbitals for the $\mathrm{C}_{2 \mathrm{v}}$ isomer, four two-center bonding (53b, 56a, 57a, 54b) orbitals for the $\mathrm{C}_{2}$ isomer, and two two-center bonding (46a" and 64a') and two three-center 
bonding (47a" and 63a') orbitals for the $\mathrm{C}_{\mathrm{s}}$ quasi-fullerene. In order to investigate the importance of configurations generated by excitations from the three-center bonding $9 \mathrm{e}_{\mathrm{lg}}$ orbitals (HOMO- 1 and HOMO-2) of the $\mathrm{D}_{6 \mathrm{~h}}$ isomer, an extended active space that included 6 electrons in 6 orbitals was also used. In MP2 and MRMP2 calculations the carbon 1s-orbitals were kept frozen; all excitations from other occupied orbitals to all valence orbitals were included in the calculations. All MRMP2 calculations were done using the parallel implementation of this code $[17,24,25]$ on a cluster of Compaq XP1000 workstations. The GAMESS suite of programs was used for all calculations [26-28].

\section{Results and discussion}

Since a Jahn-Teller distortion of the $\mathrm{D}_{6 \mathrm{~h}}$ isomer was reported, full geometry optimizations of the singlet structure were performed in $D_{6 h}, C_{6 v}$ and $D_{6}$ symmetries. The relative energies of the distorted structures are presented in Table 1. The PM3 and HF energies of this isomer clearly indicate that distortions to lower symmetry are favored. The structure with $\mathrm{C}_{6 \mathrm{v}}$ symmetry has the lowest energy at both PM3 and HF levels of theory. Diagonalization of the hessian illustrates that even the $\mathrm{C}_{6 \mathrm{v}}$ geometry corresponds to a transition state (one imaginary frequency), and a further distortion to a still lower symmetry group must take place. On the other hand, the B3LYP geometry optimizations initiated from the HF optimized geometries converged to a $D_{6 \mathrm{~h}}$ structure from all three symmetry groups. In addition, the B3LYP hessian calculation showed that this $\mathrm{D}_{6 \mathrm{~h}}$ structure has no imaginary frequency and hence corresponds to a minimum on the potential energy surface.

In order to clarify this picture single point MP2 calculations were performed at the $D_{6 h}, C_{2 v}$ and $D_{6} H F$ geometries. The MP2 energy of the $D_{6 h}$ structure is about $200 \mathrm{~kJ} / \mathrm{mol}$ lower than the energies of the distorted structures. The analysis of the MP2 natural orbital occupation numbers (NOON), that show the formal number of electrons in each molecular orbital, suggests that the $D_{6 h}$ wave function has significant multireference character. This is, not true for the $\mathrm{C}_{6 \mathrm{v}}$ and $\mathrm{D}_{6}$ structures. This may be a key point for understanding the JahnTeller distortion predicted by the PM3, HF and (according to some reports [3]) the B3LYP levels of theory. Since HF does not include electron correlation at all and PM3 and hybrid 
HF/DFT do not treat correlation effects systematically, these methods are not able to take into account the diradical character of the wave function. They therefore lower the energy of the $D_{6 h}$ isomer by distorting it to a lower symmetry group. As will be shown below, the dynamic part of the electron correlation is also important, and the B3LYP method is not able to recover it sufficiently. In other words the artificial distortion of the geometry is caused by an oversimplified form of the wave function. There are many examples of such wave function symmetry breaking resulting in symmetry breaking of the molecular geometry [29]. Interestingly, the restricted (RHF) $D_{6 h}$ singlet wave function is not stable relative to distortion to an unrestricted (UHF) wave function. The UHF wave function is very spin contaminated, but there is no geometry distortion from the $\mathrm{D}_{6 \mathrm{~h}}$ symmetry group at the UHF level of theory. This is consistent with the foregoing analysis that a correlated wave function is required for this system, and that a multi-reference description may be important.

For the triplet $\mathrm{D}_{2 \mathrm{~d}}$ isomer, which has a doubly degenerate HOMO, the Jahn-Teller theorem dictates that a distortion to a lower symmetry group must take place. Since the firstorder Jahn-Teller distortion should lift the degeneracy, the simplest distortion would be to the $\mathrm{C}_{2 v}$ or $\mathrm{D}_{2}$ subgroups. But according to the PM3, HF and B3LYP methods, there is a distortion to the even lower $\mathrm{C}_{\mathrm{s}}$ symmetry group (Table 1). On the other hand, MP2 suggests that the $C_{s}$ structure has the highest energy and $D_{2}$ the lowest energy. If the distortion to the $\mathrm{C}_{2 \mathrm{v}}$ or $\mathrm{D}_{2}$ structures is caused by a first-order Jahn-Teller effect, then a second-order JahnTeller effect could be responsible for the further distortion to $C_{s}$ symmetry. However, it is likely that, as for singlet $D_{6 h}$, the inadequate treatment of electron correlation causes the energy lowering due to a non-physical distortion of the system. This non-physical distortion allows the mixing of orbitals that in $\mathrm{D}_{2 \mathrm{~d}}$ symmetry belong to different irreducible representations.

Table 2 contains the energies of the singlet and triplet states of five $C_{36}$ isomers relative to the $\mathrm{D}_{2 \mathrm{~d}}$ singlet. In general the PM3, RHF and B3LYP relative energies are consistent with previously published results. All three methods predict that the $\mathrm{D}_{2 \mathrm{~d}}$ singlet structure has the lowest energy. The non-distorted $D_{6 h}$ singlet has the highest energy among singlet and triplet states of all isomers. According to all three methods, the $\mathrm{D}_{6 \mathrm{~h}}$ triplet 
structure is about $20 \mathrm{~kJ} / \mathrm{mol}$ higher on the energy scale than the $\mathrm{D}_{2 \mathrm{~d}}$ singlet isomer. Surprisingly, the $\mathrm{C}_{2 \mathrm{v}}$ and $\mathrm{C}_{2}$ isomers are almost as stable as the $\mathrm{D}_{2 \mathrm{~d}}$ isomer.

The distribution of the isomers on the energy scale becomes completely different after taking electron correlation effect into account. Even single reference MP2 indicates that the $D_{6 \mathrm{k}}$ singlet is more then $150 \mathrm{~kJ} / \mathrm{mol}$ lower in energy than the singlet $D_{2 \mathrm{~d}}$ isomer. The MP2 $D_{6 h}$ triplet is $64 \mathrm{~kJ} / \mathrm{mol}$ lower in energy than the $D_{2 d}$ singlet. Even the MP2 $D_{2 d}$ triplet is 25 $\mathrm{kJ} / \mathrm{mol}$ below the $\mathrm{D}_{2 \mathrm{~d}}$ singlet. These results demonstrate that dynamic electron correlation is essential for the correct prediction of the relative energies of the isomers. This also indicates that, at least in this case, B3LYP is not able to sufficiently recover the dynamic part of the correlation energy.

The relative energies of the $\mathrm{C}_{36}$ fullerenes calculated by the CASSCF method indicate that the nondynamic part of the electron correlation is also important. Even though CASSCF predicts the singlet $D_{2 d}$ isomer to have the lowest energy, the difference between its energy and energy of the $D_{6 \mathrm{~h}}$ singlet is reduced from the $\mathrm{HF}$ value of $108 \mathrm{~kJ} / \mathrm{mol}$ to $25 \mathrm{~kJ} / \mathrm{mol}$. The CASSCF NOON (Table 3) clearly indicate that the singlet $D_{6 \mathrm{~h}}$ wave function has multireference character. Since 1.37 electrons occupy the HOMO and 0.61 electrons occupy the LUMO, the singlet state of the $\mathrm{D}_{6 \mathrm{~h}}$ isomer has significant diradical character. The CASSCF calculations on the $D_{6 h}$ singlet structure with the extended $(6,6)$ active space shows that the NOON of degenerate HOMO-1 and HOMO-2 orbitals are 1.997. This indicates that excitations from these orbitals play a negligible role. Since the NOON of the other singlet isomers are close to either 2 or 0 the wave functions of these isomers can be well described by single-reference wave functions. Since there is only one CI determinant for the $D_{6 h}$ and $\mathrm{C}_{2 \mathrm{v}}$ triplet states, the CASSCF calculations are equivavalent to HF ones. The relative energies calculated with multireference perturbation theory, based on CASSCF wave functions are presented in the last column of Table 2. The MRMP2 relative energies of the isomers are in good qualitative agreement with the MP2 results. The $\mathrm{D}_{6 \mathrm{~h}}$ singlet structure is $183 \mathrm{~kJ} / \mathrm{mol}$ lower than the $\mathrm{D}_{2 \mathrm{~d}}$ singlet structure. The $\mathrm{D}_{2 \mathrm{~d}}$ triplet is $91 \mathrm{~kJ} / \mathrm{mol}$ lower in energy than the singlet structure of this isomer. 


\section{Summary}

This paper demonstrates the importance of electron correlation effects in the study of the relative stabilities of the isomers of $\mathrm{C}_{36}$ fullerene. According to the PM3, HF and B3LYP methods, the $D_{2 d}$ isomer is more stable than the $D_{6 h}$ isomer on the singlet potential energy surface. In contrast, the relative energies calculated with MRMP2 indicate that the $\mathrm{D}_{6 \mathrm{~h}}$ singlet is $183 \mathrm{~kJ} / \mathrm{mol}$ lower in energy than the $\mathrm{D}_{2 \mathrm{~d}}$ singlet. The $\mathrm{D}_{6 \mathrm{~h}}$ and $\mathrm{D}_{2 \mathrm{~d}}$ triplet structures are 148 $\mathrm{kJ} / \mathrm{mol}$ and $91 \mathrm{~kJ} / \mathrm{mol}$ lower than the $\mathrm{D}_{2 \mathrm{~d}}$ singlet. The wave function of the $\mathrm{D}_{6 \mathrm{~h}}$ singlet has significant diradical character. The second-order Jahn-Teller distortion of the $\mathrm{D}_{6 \mathrm{~h}}$ isomer reported by several authors appears to be artificial due to the oversimplified form of the wave function used in these calculations. By including electron correlation, the distortion is eliminated.

The diradical character of the $\mathrm{D}_{6 \mathrm{~h}}$ isomer is probably one reason that many attempts to synthesize the pure form of $\mathrm{C}_{36}$ fullerene have not been successful. However, it is still possible to imagine the formation of a stable covalent crystal of $\mathrm{C}_{36}$ cages [1]. In addition, it has been shown recently $[30,31]$ that the $\mathrm{C}_{36}$ fullerene can be stabilized by attaching to it two or more hydrogen atoms.

\section{Acknowledgments}

The authors thank Dr. Mike Schmidt for helpful discussions. The calculations in this work were performed in part on an IBM workstation cluster made possible by grants from IBM in the form of a Shared University Research grant and the United State Department of Energy. Partially the work was supported by Grant \#3l of Federal Russian Program "Integration" "Creation of a center of computer clusters and distributed calculation systems of joint use".

\section{References}

[1] C. Piskoti, J. Yarger, A. Zettl, Nature (London) 393 (1998) 771. 
[2] P.W. Fowler, D.E. Manolopoulos, An Atlas of Fullerenes, Clarendon, Oxford, 1995.

[3] Z. Slanina, F. Uhlik, X. Zhao, E. Osawa, J. Chem. Phys. 113, (2000) 4933.

[4] J.C. Grossman, M. Cote, S.G. Louie, M.L. Cohen, Chem. Phys. Lett. 284 (1998) 344.

[5] E. Halac, E. Burgos, H. Bonadeo, Chem. Phys. Lett. 299 (1999) 64.

[6] M.N. Jagadeesh, J. Chandrasekhar, Chem. Phys. Lett. 305 (1999) 298.

[7] A. Ito, T. Monobe, T. Yoshii, K. Tanaka, Chem. Phys. Lett. 328 (2000) 32.

[8] Z. Chen, H. Jiao, A. Hirsch, W. Thiel, Chem. Phys. Lett. 329 (2000) 47.

[9] L. Yuan, J. Yang, K. Deng, Q. Zhu, J. Phys. Chem. A. 104 (2000) 6666.

[10] Z. Slanina, X. Zhao, E. Osawa, Chem. Phys. Lett. 290 (1998) 311.

[11] Z. Slanina, X. Zhao, E. Osawa, Electrochemical Society Proceedings 99-12 (1999) 682.

[12] Z. Slanina, F. Uhlik, X. Zhao, E. Osawa, J. Chem. Phys. 113 (2000) 4933.

[13] P.W. Fowler, T. Heine, K.M. Rogers, J.P.B. Sandall, G. Seifert, F. Zerbetto, Chem. Phys. Lett. 300 (1999) 369.

[14] P.W. Fowler, D. Mitchell, F. Zerbetto, J. Am. Chem. Soc. 121 (1999) 3218.

[15] B.O. Roos, Adv. Chem. Phys. 69 (1987) 399.

[16] C. Moller, M.S. Plesset, Phys. Rev. 46 (1934) 618.

[17] K. Hirao, Chem. Phys. Lett. 190 (1992) 374.

[18] J.J.P. Stewart, J. Computer-Aided Molecular Design, 4 (1990) 1.

[19] A.D. Becke, J. Chem. Phys. 98 (1993) 5648.

[20] P.J. Stephens, F.J. Devlin, C.F. Chablowski, M.J. Frisch, J. Phys. Chem. 98 (1994) 11623.

[21] R.H. Hertwig, W. Koch, Chem. Phys. Lett. 268 (1997) 345.

[22] T.J. Lee, D. Jayatilaka, Chem. Phys. Lett. 201 (1993) 1.

[23] T.J. Lee, A.P. Rendell, K.G. Dyall, D. Jayatilaka, J. Chem. Phys. 100 (1994) 7400.

[24] H. Nakano, J. Chem. Phys. 99 (1993) 7983.

[25] H. Umeda, S. Koseki, U. Nagashima, M.W. Schmidt, J. Comput. Chem. 22 (2001) 1243.

[26] M.W. Schmidt, K.K. Baldridge, J. A. Boatz, S.T. Elbert, M.S. Gordon, J.H. Jensen, S. Koseki, N. Matsunaga, K.A. Nguyen, S.J. Su, T.L. Windus, M. Dupuis, J.A. Montgomery, J. Comp.Chem. 14 (1993) 1347. 
[27] G.D. Fletcher, M.W. Schmidt, M.S. Gordon, Adv. Chem. Phys. 110 (1999) 267.

[28] G.D. Fletcher, M.W. Schmidt, B.M. Bode, M.S. Gordon, Comput. Phys. Commun. 128 (2000) 190.

[29] E.R. Davidson, V.T. Borden, J. Phys. Chem. 87 (1983) 4783.

[30] A. Koshio, M. Inakuma, T. Sugai, H. Shinohara, J. Am. Chem. Soc. 122 (2000) 398.

[31] A. Koshio, M. Inakuma, Z. W. Wang, T. Sugai, H. Shinohara, J. Phys. Chem. B. 104 (2000) 7908 . 
Table 1. Energies ( $\mathrm{kJ} / \mathrm{mol})$ of the $\mathrm{D}_{6 \mathrm{~h}}$ singlet isomer and the $\mathrm{D}_{2 \mathrm{~d}}$ triplet isomer distorted to lower symmetry groups, presumably by a second-order Jahn-Teller effect. For the $\mathrm{D}_{6 \mathrm{~h}}$ singlet state the energies are relative to the undistorted structure. For the $D_{2 \mathrm{~d}}$ triplet state the energies are relative to the $D_{2}$ structure (the $D_{2}$ symmetry of the $D_{2 d}$ triplet state resulted from a firstorder Jahn-Teller effect).

\begin{tabular}{lcccc}
\hline Symmetry & PM3 & HF & B3LYP & MP2 $^{\mathbf{a}}$ \\
\hline \multicolumn{5}{c}{ Singlet $\mathrm{D}_{6 \mathrm{~s}}$ isomer } \\
$\mathrm{C}_{6 \mathrm{v}}$ & -54.0 & -27.8 & 0.0 & 203.3 \\
$\mathrm{D}_{6}$ & -30.8 & -26.6 & 0.0 & 195.0 \\
$\mathrm{D}_{6 \mathrm{~b}}$ & 0.0 & 0.0 & 0.0 & 0.0 \\
& & Triplet $\mathrm{D}_{2 \mathrm{~d}}$ isomer & & \\
$\mathrm{C}_{\mathrm{s}}$ & -58.0 & -57.3 & -6.6 & 179.8 \\
$\mathrm{C}_{2 \mathrm{v}}$ & -38.4 & -33.0 & -4.5 & 46.1 \\
$\mathrm{D}_{2}$ & 0.0 & 0.0 & 0.0 & 0.0 \\
\hline
\end{tabular}

${ }^{a}$ Geometry has been optimized at the HF level of theory. 
Table 2. Energies $(\mathrm{kJ} / \mathrm{mol})$ of the $\mathrm{C}_{36}$ isomers relative to the singlet $\mathrm{D}_{2 \mathrm{~d}}$ isomer calculated at different levels of theory.

\begin{tabular}{|c|c|c|c|c|c|c|}
\hline Isomers & PM3 & $\mathbf{H F}$ & B3LYP & $\mathbf{M P 2}^{\mathbf{a}}$ & CASSCF $^{a}$ & $\mathbf{M R M P 2}^{\mathbf{a}}$ \\
\hline \multicolumn{7}{|c|}{ Singlet states } \\
\hline $\mathrm{D}_{2 \mathrm{~d}}$ & 0.0 & 0.0 & 0.0 & 0.0 & 0.0 & 0.0 \\
\hline $\mathrm{C}_{2 \mathrm{v}}$ & 27.2 & 4.7 & 30.4 & 44.3 & -0.4 & 75.0 \\
\hline $\mathrm{C}_{\mathrm{s}}$ quasi-fullerene & 65.0 & 49.1 & 85.7 & 111.8 & 35.9 & 204.2 \\
\hline $\mathrm{C}_{2}$ & 88.9 & 24.0 & 26.8 & -2.0 & 0.7 & 41.3 \\
\hline $\mathrm{D}_{6 \mathrm{~h}}$ & 156.3 & 107.5 & 24.8 & -154.6 & 25.1 & -182.5 \\
\hline \multicolumn{7}{|c|}{ Triplet states } \\
\hline $\mathrm{D}_{2 \mathrm{~d}}\left(\mathrm{D}_{2}\right)$ & 66.6 & 48.3 & 19.9 & -24.8 & 64.0 & -90.7 \\
\hline $\mathrm{C}_{2 v}$ & 86.7 & 47.1 & 47.4 & 42.4 & 62.7 & -21.4 \\
\hline $\mathrm{C}_{\mathrm{s}}$ quasi-fullerene $\mathrm{e}^{\mathrm{b}}$ & 124.8 & 99.0 & 108.8 & 191.7 & - & - \\
\hline $\mathrm{C}_{2}^{\mathrm{b}}$ & 20.0 & 7.8 & 40.4 & 158.4 & - & - \\
\hline $\mathrm{D}_{6 \mathrm{~h}}$ & 25.3 & 18.5 & 23.3 & -64.3 & 34.1 & -148.3 \\
\hline
\end{tabular}

${ }^{\mathrm{a}}$ Geometry has been optimized at the HF level of theory.

${ }^{\mathrm{b}}$ CASSCF calculation did not converge for this electronic state. 
Table 3. Number of configurations in the CASSCF active space and natural orbital occupation numbers (NOON) of the active space orbitals.

\begin{tabular}{lcccccc}
\hline \multicolumn{1}{c}{ Isomer } & Elec. state \# of CI det. & HOMO & LUMO & LUMO+1 & LUMO+2 \\
\hline \multicolumn{7}{c}{} \\
$\mathrm{D}_{2 \mathrm{~d}}$ & ${ }^{1} \mathrm{~A}_{1}$ & 5 & 1.958 & 0.017 & 0.017 & 0.007 \\
$\mathrm{C}_{2 \mathrm{v}}$ & ${ }^{1} \mathrm{~A}_{1}$ & 5 & 1.965 & 0.021 & 0.009 & 0.005 \\
$\mathrm{C}_{\mathrm{s}}$ quasi-fullerene & ${ }^{1} \mathrm{~A}^{\prime}$ & 6 & 1.969 & 0.021 & 0.009 & 0.001 \\
$\mathrm{C}_{2}$ & ${ }^{1} \mathrm{~A}$ & 6 & 1.910 & 0.085 & 0.002 & 0.003 \\
$\mathrm{D}_{6 \mathrm{~b}}$ & ${ }^{1} \mathrm{~A}_{1 \mathrm{~g}}$ & 4 & 1.372 & 0.610 & 0.009 & 0.009 \\
$\mathrm{D}_{2 \mathrm{~d}}\left(\mathrm{D}_{2}\right)^{\mathrm{a}}$ & ${ }^{3} \mathrm{~B}_{2}$ & 2 & 1.000 & 1.000 & 0.000 & 0.000 \\
$\mathrm{C}_{2 v}$ & ${ }^{3} \mathrm{~B}_{1}$ & 1 & 1.000 & 1.000 & 0.000 & 0.000 \\
$\mathrm{C}_{\mathrm{s}}$ quasi-fullerene & ${ }^{3} \mathrm{~A}^{\mathrm{b}}$ & 4 & - & - & - & - \\
$\mathrm{C}_{2}{ }^{\mathrm{b}}$ & ${ }^{3} \mathrm{~B}$ & 4 & - & - & - & - \\
$\mathrm{D}_{6 \mathrm{~b}}$ & ${ }^{3} \mathrm{~A}_{24}$ & 1 & 1.000 & 1.000 & 0.000 & 0.000 \\
\hline
\end{tabular}

${ }^{a}$ The $\mathrm{D}_{2 \mathrm{~d}}$ triplet state is distorted to $\mathrm{D}_{2}$ symmetry as a result of first-order Jahn-Teller effect. ${ }^{b}$ CASSCF method did not converge for this electronic state. 


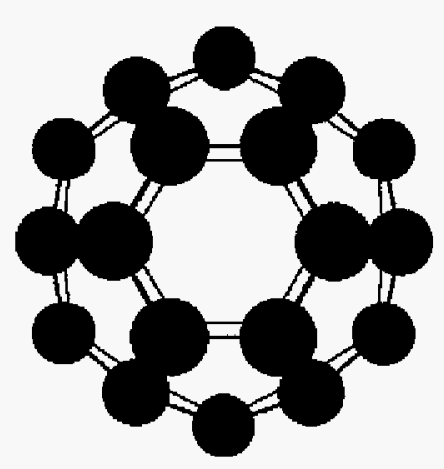

D6h

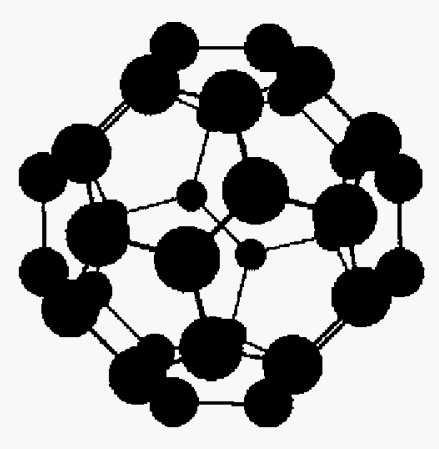

$D_{2 d}$

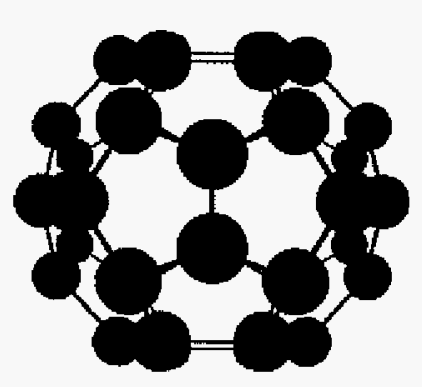

$\mathrm{C}_{2 \mathrm{v}}$

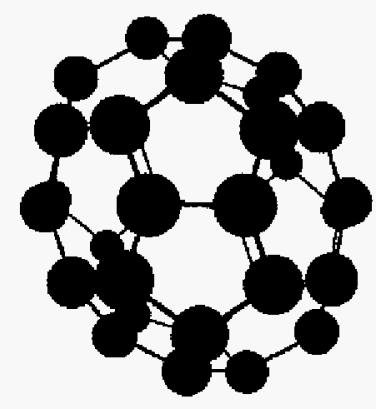

$\mathrm{C}_{2}$

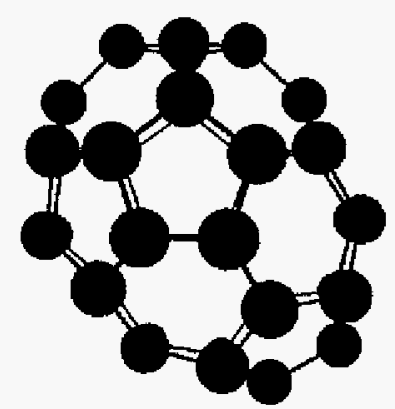

$C_{s}$ quasi-fullerine

Figure 1. Five lowest energy isomers of $C_{36}$ fullerene at B3LYP/6-31G* level of theory. 

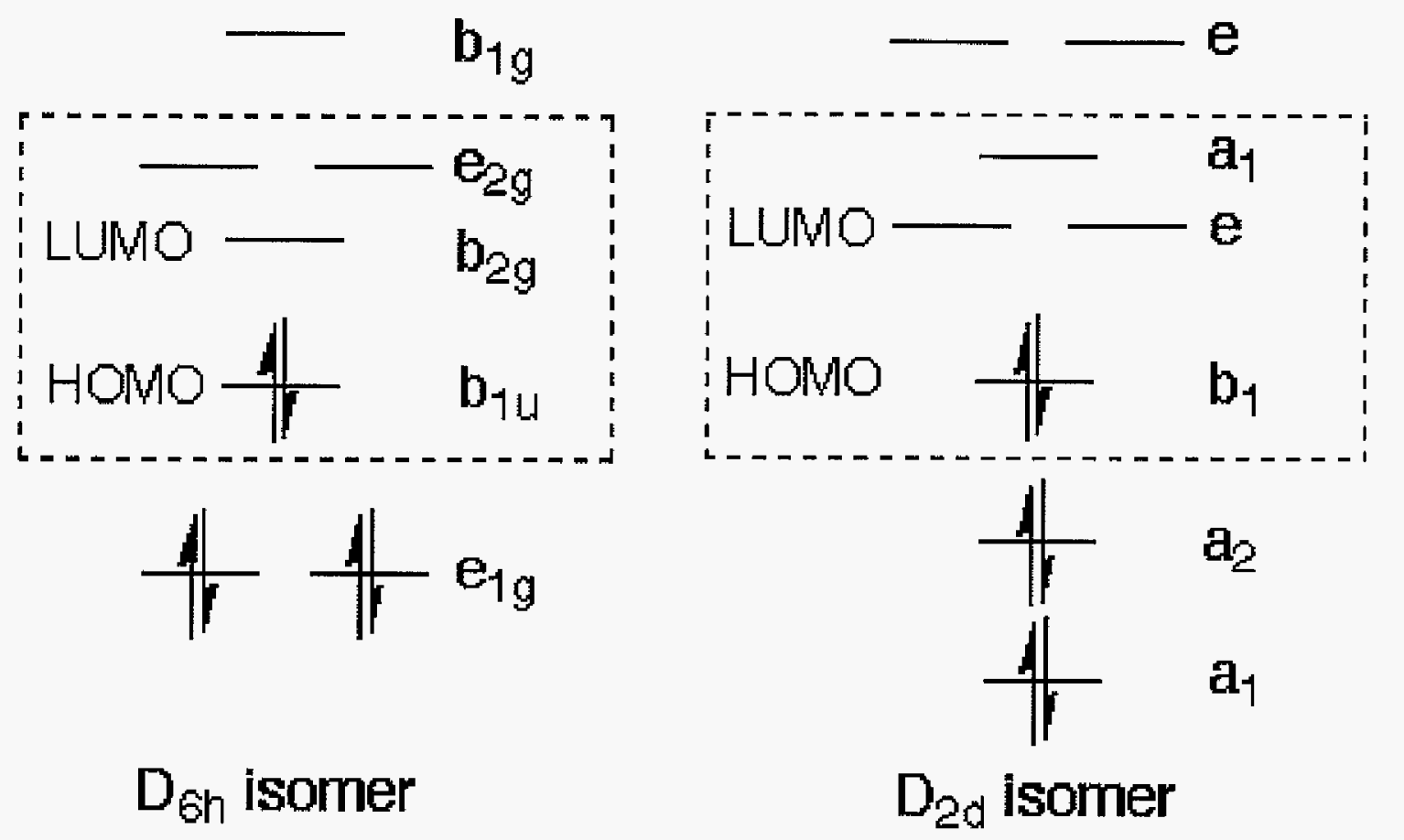

Figure 2. CASSCF active spaces for the $\mathrm{D}_{6 \mathrm{~h}}$ and $\mathrm{D}_{2 \mathrm{~d}}$ isomers. The active spaces are contained in the dashed boxes. 


\title{
CHAPTER 5. EFFECTS OF STRONG ELECTRON CORRELATIONS IN Ti $\mathrm{i}_{8} \mathrm{C}_{12}$ MET-CAR
}

Taken from a paper to be submitted to the Journal of Chemical Physics.

Sergey A. Varganov, Mark S. Gordon

\begin{abstract}
The results of multireference configuration interaction (MRCI) with single and double excitations and single reference coupled cluster $(\operatorname{CCSD}(\mathrm{T}))$ calculations on $\mathrm{Ti}_{8} \mathrm{C}_{12}$ metallocarbohedrene (Met-Car) are reported. The distortions of the $T_{d}$ structure to $D_{2 d}$ and $\mathrm{C}_{3 \mathrm{v}}$ structures due to the Jahn-Teller effect are studied with multireference methods. It is shown that the $\mathrm{Ti}_{8} \mathrm{C}_{12}$ wave function has significant multireference character. The choice of the active space for multireference self-consistent field (MCSCF) calculations is discussed. The failure of multireference perturbation theory with a small active space is attributed to multiple intruder states. A new, novel type of MCSCF calculation, ORMAS (Occupation Restricted Multiple Active Spaces) with a large active space are carried out for several electronic states of $\mathrm{Ti}_{8} \mathrm{C}_{12}$. According to $\operatorname{CCSD}(\mathrm{T})$ the ${ }^{1} \mathrm{~A}_{1}$ and ${ }^{3} \mathrm{~B}_{1}$ states with distorted $\mathrm{D}_{2 \mathrm{~d}}$ (nearly $\mathrm{T}_{\mathrm{d}}$ ) symmetry are the lowest energy states of $\mathrm{Ti}_{8} \mathrm{C}_{12}$. These two states are predicted to be less then $3 \mathrm{kcal} / \mathrm{mol}$ apart. The ${ }^{1} \mathrm{~A}_{1}$ and ${ }^{3} \mathrm{~A}_{1}$ states in $\mathrm{C}_{3 \mathrm{v}}$ symmetry are also possible candidates for the ground state of $\mathrm{Ti}_{8} \mathrm{C}_{12}$. Predictions of the $\mathrm{Ti}_{8} \mathrm{C}_{12}$ ionization potential with different ab-initio methods are presented.
\end{abstract}

\section{Introduction}

Metal-carbon clusters with $\mathrm{M}_{8} \mathrm{C}_{12}$ composition, where $M$ is a metal atom, were discovered by Castleman almost 15 years ago. ${ }^{1}$ Such clusters seem to be more thermodynamically stable than small carbide precursors with $\mathrm{M}_{\mathrm{x}} \mathrm{C}_{\mathrm{x}}$ stoichiometry. ${ }^{2}$ Potentially, met-cars can find applications as new catalysts and high-temperature resistant materials. The $\mathrm{Ti}_{8} \mathrm{C}_{12}$ cluster is probably the most studied met-car. However, after many 
years of work, the geometry and the ground electronic state of $\mathrm{Ti}_{8} \mathrm{C}_{12}$ have not been clearly established. The originally proposed $T_{h}$ structure (Fig. 1) was found to be unstable with respect to distortion. ${ }^{3}$ However, it was shown that a cluster with $\quad T_{1}$ symmetry can be distorted to several topologically different structures by rotating the $\mathrm{C}_{2}$ fragments. ${ }^{2}$ According to most density functional theory (DFT) calculations the most stable $\mathrm{Ti}_{8} \mathrm{C}_{12}$ geometry has $T_{d}$ topology (Fig. 1) with the possibility of Jahn-Teller distortion to lower symmetry structures. ${ }^{4,5}$ The most recent DFT calculations predict the $\mathrm{D}_{2 \mathrm{~d}}$ and $\mathrm{C}_{3 \mathrm{v}}$ structures to be almost isoenergetic, with the $\mathrm{C}_{3 \mathrm{v}}$ structure slightly more stable. ${ }^{6}$ However, in most DFT studies distortion to even lower symmetry (down to $C_{1}$ ) structures is predicted. ${ }^{6,7}$

A serious problem with using density functional theory methods to study Jahn-Teller distortions is the fact that DFT cannot be used to calculate the correct electron structure of the ground state of the undistorted $T_{d}$ structure. In most DFT implementations a single determinant wave function is used. Since most DFT calculations predict the highest occupied molecular orbital (HOMO) of the $T_{d}$ structure to be triply degenerate and occupied by two electrons, the singlet state wave function (and therefore the electron density and any properties) must be represented by a linear combination of at least three determinants. In addition, because the ${ }^{1} \mathrm{E}$ ground state is doubly degenerate, one must average the two components of the wave function. The same is true for the triplet electronic state, except the state averaging has to be done over three components of the wave function. It can be argued that since the Jahn-Teller effect lifts the degeneracy of the ground state (and one-electron orbitals) DFT can safely be used to study the distorted structures. However, if the distortion is small there are still significant contributions from all three determinants to the total wave function, so calculating the electron density from a single determinant wave function is a questionable approach. Therefore, the results obtained from such DFT studies of $\mathrm{Ti}_{8} \mathrm{C}_{12}$ structures with degenerate or nearly degenerate states, for example, in $\mathrm{D}_{2 \mathrm{~d}}$ or $\mathrm{C}_{3 \mathrm{v}}$ symmetry that arise from Jahn-Teller distortion of the $T_{d}$ structure, could be suspect.

The purpose of this study is to determine the ground state and the relative energies of several low lying excited states of $\mathrm{Ti}_{8} \mathrm{C}_{12}$ using ab-initio methods. The $\mathrm{T}_{\mathrm{d}}$ structure and its distorted $\mathrm{D}_{2 \mathrm{~d}}$ and $\mathrm{C}_{3 \mathrm{v}}$ structures are considered. The study of Jahn-Teller distortions from the $T_{d}$ structure to $D_{2 d}$ and $C_{3 v}$ geometries is accomplished using complete active space self 
consistent field (CASSCF) ${ }^{8}$ wave functions, augmented by multireference configuration interaction $(\mathrm{MRCI})^{9}$ with single and double excitations. The distorted structures are also studied with coupled cluster theory, including singles and doubles with perturbative triples $(\operatorname{CCSD}(\mathrm{T}))^{10}$. Some perturbation theory and DFT calculations were carried out as well. The $\mathrm{Ti}_{8} \mathrm{C}_{12}$ ionization potential is calculated using both $\operatorname{CCSD}(\mathrm{T})$ and $\mathrm{MRCI}$.

\section{Computational methods}

All geometries were initially optimized with the Hartree-Fock (HF) method. Optimized HF geometries were used as an initial guess for DFT, CASSCF and MøllerPlesset second order perturbation theory (MP2) ${ }^{11}$ geometry optimizations. For CASSCF geometry optimization of $\mathrm{T}_{d}$ degenerate states, the HF geometry of single reference ${ }^{5} \mathrm{~A}_{2}$ state was used as the starting structure. The energy gradients for all geometry optimizations, except open-shell MP2 calculations, were evaluated analytically. For open-shell MP2 geometry optimizations, the gradients were calculated numerically. The $\operatorname{CCSD}(\mathrm{T})$ energies were calculated at optimized HF and MP2 geometries. The MRCI calculations were done at CASSCF optimized geometries. The HF, CASSCF and DFT Hessians (matrices of energy second derivatives with respect to nuclear coordinates) were calculated by numerical differentiation of analytic energy gradients using single differencing.

To avoid spin contamination in the wave function, restricted open-shell methods were used for all single-reference calculations. The MP2 open-shell calculations were done with Z-averaged second-order perturbation theory $(\mathrm{ZAPT} 2)^{12}$. For open-shell coupled cluster calculations the restricted coupled cluster $\operatorname{RCCSD}(T)^{13}$ method was used. In all single reference methods the restricted open-shell Hartree-Fock (ROHF) wave function was used as the zero-order approximation. In the DFT calculations a spin-restricted wave function and hybrid $\mathrm{B} 3$ LYP ${ }^{14}$ functional were used. In all post-Hartree-Fock calculations the 1s electrons of carbon and $1 \mathrm{~s} 2 \mathrm{~s} 2 \mathrm{p} 3 \mathrm{~s} 3 \mathrm{p}$ electrons of Ti were not correlated. All calculations were done with the $6-31 \mathrm{G}(\mathrm{d})$ basis set ${ }^{15}$. Hartree-Fock and density functional calculations were carried out using the full symmetry of the respective point symmetry group. All post-Hartree-Fock calculations were done in highest Abelian subgroup of actual symmetry group. The 
GAMESS suite of programs ${ }^{16}$ was used for all HF, MCSCF, MP2 and B3LYP calculations. The CCSD(T) and MRCI as well as MP3 and MP4 calculations were done with MOLPRO ${ }^{17}$.

\section{Results and discussion}

\section{A. Model for $\mathbf{T i}_{8} \mathrm{C}_{12}$ electron structure}

The one-electron molecular orbitals can provide significant insight into the electronic structure of $\mathrm{Ti}_{8} \mathrm{C}_{12}$. A detailed analysis can be found elsewhere ${ }^{7}$. Here, it is sufficient to note that the 30 lowest energy valence orbitals, occupied by 60 electrons, are centered exclusively on the $\mathrm{C}_{2}$ fragments, with no significant contribution from $\mathrm{Ti}$ atomic orbitals. The remaining 10 occupied valence orbitals mostly consist of linear combinations of Ti d-orbitals. The simple orbital diagram proposed by Lin and Hall ${ }^{18}$ provides a useful qualitative description of the electron structure of $\mathrm{Ti}_{8} \mathrm{C}_{12}$ (Fig. 2). According to this diagram two d-orbitals on each $\mathrm{Ti}$ atom participate in the formation of ionic Ti-C bonds; the other three d-orbitals from each $\mathrm{Ti}$ atom are available for $\mathrm{Ti}-\mathrm{Ti}$ interactions. The interactions among these $24 \mathrm{Ti}$ orbitals produces nine bonding orbitals, which accommodate 18 of the 20 remaining Ti d-electrons, nine nonbonding and six antibonding orbitals. This picture is supported by HF calculations on $\mathrm{Ti}_{8} \mathrm{C}_{12}{ }^{2+}$, which has a large HOMO-LUMO gap ${ }^{7}$. Therefore, in a one-electron orbital picture, the main question regarding the electronic structure of $\mathrm{Ti}_{8} \mathrm{C}_{12}$ is which orbitals do the last two valence electrons occupy? The answer to this question would not only determine the ground electronic state of $\mathrm{Ti}_{8} \mathrm{C}_{12}$, but would also determine the symmetry of this cluster. For example, if these two electrons occupy one of the triply degenerate orbitals the $T_{d}$ structure will be subject to the first-order Jahn-Teller effect and should therefore distort to $\mathrm{D}_{2 \mathrm{~d}}$ or $\mathrm{C}_{3 \mathrm{v}}$, or perhaps lower, symmetry. Such a distortion would remove the degeneracy of the ground state. If the two electrons occupy a nondegenerate orbital the met-car would presumably retain $\mathrm{T}_{\mathrm{d}}$ symmetry, assuming there is no second-order Jahn-Teller effect. It is important to point out that due to the large distances between Ti atoms the interactions among the $24 \mathrm{Ti}$ orbitals is weak. As a result the nonbonding and even the antibonding orbitals are almost degenerate.

There are several ways to avoid Jahn-Teller distortion at the HF level of theory (Fig. 3). The last two valence electrons can be placed in the $5 \mathrm{a}_{1}$ orbital, resulting in a ${ }^{\mathrm{I}} \mathrm{A}_{1}$ 
electronic state. However, in reality the lowest energy $\mathrm{HF}$ state is ${ }^{5} \mathrm{~A}_{2}$. In this state the four unpaired electrons occupy the $4 a_{1}$ and $7 t_{2}$ orbitals. From Fig. 3 it is clear that the $4 a_{1}$ and $7 t_{2}$ orbitals are centered on the same $\mathrm{Ti}$ atoms and are very similar in shape. This shape similarity produces large exchange integrals between these orbitals, resulting in an energy lowering due to this strong exchange interaction. The relative energies of low-spin states cannot be calculated correctly with single determinant wave functions. A CASSCF wave function, with an active space of four electrons in four orbitals $\left(4 \mathrm{a}_{1}, 7 \mathrm{t}_{2}\right)$, predicts that the doubly degenerate ${ }^{1} \mathrm{E}$ state is the ground state with ${ }^{3} \mathrm{~T}_{1}$ and ${ }^{5} \mathrm{~A}_{2} 2.4$ and $8.0 \mathrm{kcal} / \mathrm{mol}$ higher in energy, respectively ${ }^{19}$.

If the last two valence electrons occupy one or two of the triply degenerate $7 \mathrm{t}_{2}$ orbitals, the $T_{d}$ structure must distort to $D_{2 d}$ or $C_{3 v}$ symmetry due to the Jahn-Teller effect. The early calculations of Rohmer et al. showed that at the HF level of theory the singlet states of the distorted structures are higher in energy than the $T_{d}{ }^{5} A_{2}$ state ${ }^{20}$. However, more recent MP2 calculations predicted that the distorted structures are lower in energy than the non-distorted one. ${ }^{7}$

Several singlet and triplet $\mathrm{HF}$ solutions for distorted $\mathrm{Ti}_{8} \mathrm{C}_{12}$ structures are very close to each other in energy. It is important to understand for a given spin and symmetry, there is only one correct HF solution. Higher energy solutions for the same spin and symmetry do not correspond to excited states, since orthogonality cannot be maintained with the lower energy solution. Therefore, the higher energy solutions are simply approximations to the lowest energy HF solution. Usually, the one correct HF solution is significantly lower in energy than other solutions and it is often difficult to converge HF to a higher energy solution. However, for $\mathrm{Ti}_{8} \mathrm{C}_{12}$ there are several quasi-degenerate HF solutions. Although the correct HF solution is clearly the one with lowest energy, it is not obvious which is the best choice for a starting reference wave function for post-HF calculations.

In the following discussion different HF solutions for the same electronic state are distinguished by specifying the highest occupied molecular orbital (HOMO) in the $T_{d}$ structure. For example, consider the $\mathrm{T}_{\mathrm{d}}{ }^{1} \mathrm{E}$ and ${ }^{3} \mathrm{~T}_{1}$ states that arise from placing the last two valence electrons in the $7 t_{2}$ orbital. Upon distortion from $T_{d}$ to $D_{2 d}$, the triply degenerate $7 t_{2}$ orbital splits into a nondegenerate $b_{2}$ orbital and a doubly degenerate e orbital (Fig. 4). 
Placing two electrons in the $b_{2}$ orbital produces a ${ }^{1} A_{1}\left(7 t_{2}\right)$ state. One can also produce the higher spin ${ }^{3} \mathrm{~A}_{2}\left(7 \mathrm{t}_{2}\right)$ state and the low spin singlet state by putting these two electrons in an $\mathrm{e}$ orbital. Indeed, one can obtain alternative HF solutions by placing two electrons in the $T_{d} 4 t_{l}$ orbital (Fig. 4). Upon distortion to $D_{2 d}$ symmetry, the $4 t_{1}$ orbital splits to the $a_{2}$ and e orbitals. Clearly, these $a_{2}$ and e orbitals can produce the same set of electronic states as produced by the $7 t_{2} \rightarrow b_{2}$, e orbitals. 'Mixed' states are also possible. For example, the high-spin ${ }^{3} \mathrm{~B}_{1}\left(7 \mathrm{t}_{2} 4 \mathrm{t}_{1}\right)$ state can be produced if one electron occupies the $\mathrm{b}_{2}$ orbital (component of $7 \mathrm{t}_{2}$ ) and the second electron occupies $a_{2}$ orbital (component of $4 t_{1}$ orbital) (Fig.4). In other words one must consider all states that can be produced as a direct product of states generated from the $7 \mathrm{t}_{2}{ }^{2}$ and $4 \mathrm{t}_{1}{ }^{2}$ configurations.

The existence of several competing HF solutions is an indication of strong electron correlations in $\mathrm{Ti}_{8} \mathrm{C}_{12}$. It is clear that a correct description of the electronic structure of $\mathrm{Ti}_{8} \mathrm{C}_{12}$ requires the use of a wave function that includes all such HF solutions (determinants). Two ways to attack this problem are discussed here. In the first approach a zeroth-order wave function is produced by fully correlating several electrons in a relatively small orbital subspace (active space) using the CASSCF method, to obtain what is often called the nondynamic correlation. This approach is expected to reduce the order of the excitations needed in post-CASSCF methods to obtain accurate energies. Multireference configuration interaction with singles and doubles (MRCI(SD)) is used to correlate electrons outside the CASSCF active space, to obtain the dynamic correlation. The energies of the $T_{d}{ }^{l} E$ and ${ }^{3} T_{1}$ states were calculated by state averaging two and three degenerate states, respectively.

In the second approach $\operatorname{CCSD}(\mathrm{T})$ calculations were done using several different $\mathrm{HF}$ solutions as zeroth-order wave functions. The interesting question here is whether, by using a high enough order of excitations, one can arrive at the same, or almost the same, energies using different HF solutions as references. Of course, in the limit of full configuration interaction, which includes all possible orders of excitations in a given basis set, the result cannot depend on the choice of zeroth-order wave function. 


\section{B. Multireference calculations}

The CASSCF active space for the $T_{d}$ structure is chosen to be the same as in previous studies ${ }^{19}$ and consists of four electrons in four orbitals $\left(4 \mathrm{a}_{1}\right.$ and $7 \mathrm{t}_{2}$ ). At the CASSCF level the ${ }^{1} \mathrm{E}$ and ${ }^{3} \mathrm{~T}_{1}$ states are, respectively, 8.7 and $5.7 \mathrm{kcal} / \mathrm{mol}$ lower in energy than the ${ }^{5} \mathrm{~A}_{2}$ state (Table 1). In the lowest-order approximation only the 20 electrons that occupy the Ti d-like molecular orbitals (Fig. 2) were correlated. At the MRCI level with 20 correlated electrons the aforementioned energy differences increase to 14.4 and $9.5 \mathrm{kcal} / \mathrm{mol}$ respectively. Adding the Davidson correction (MRCI+Q) increases these relative energies to 17.3 and 11.5 $\mathrm{kcal} / \mathrm{mol}$. These energies are in good agreement with previously reported results ${ }^{19}$. Since it was discussed previously that correlating only 20 electrons sometimes may not be sufficient to obtain quantitative results ${ }^{7}$, and correlating all 80 valence electrons at the $\mathrm{MRCI}$ (SD) level is currently not computationally feasible, $\mathrm{MRCI}(\mathrm{SD})$ calculations were performed with 56 correlated valence electrons that occupy the highest energy orbitals. For the three $T_{d}$ states of interest, correlating 56 vs. 20 electrons has a relatively small effect on the predicted relative energies. As shown in Table 1, the MRCI(SD)+Q calculations with 56 correlated electrons predicts the ${ }^{1} \mathrm{E}$ and ${ }^{3} \mathrm{~T}_{1}$ states to be 15.5 and $10.4 \mathrm{kcal} / \mathrm{mol}$, respectively, below ${ }^{5} \mathrm{~A}_{2}$.

Since the ${ }^{1} E$ ground state in $T_{d}$ symmetry is doubly degenerate, it must Jahn-Teller distort to lower symmetry structures. At the MRCI(SD)+Q level of theory the ${ }^{1} A_{1}\left(D_{2 d}\right)$ structure is only $2.2 \mathrm{kcal} / \mathrm{mol}$ lower in energy than the ${ }^{1} \mathrm{E}\left(\mathrm{T}_{\mathrm{d}}\right)$ structure. The natural orbital occupation numbers (NOON) (Table 2) show significant multireference character for both the $T_{d}$ and distorted structures. That is, several NOON deviate significantly from the integer values that one obtains from a single determinant description. $\mathrm{A} \mathrm{C}_{3 \mathrm{v}}$ structure was not found for the singlet electronic state. The CASSCF geometry optimization in Cs symmetry (lowest Abelian subgroup of $\mathrm{C}_{3 \mathrm{v}}$ group), using the Hartree-Fock $\mathrm{C}_{3 \mathrm{v}}$ geometry as an initial guess, converged to the $\mathrm{D}_{2 \mathrm{~d}}$ structure. The CASSCF Hesssian calculations indicate that both ${ }^{1} \mathrm{~A}_{1}$ and ${ }^{3} \mathrm{~A}_{2} \mathrm{D}_{2 \mathrm{~d}}$ states correspond to minima on the $\mathrm{Ti}_{8} \mathrm{C}_{12}$ potential energy surface. The bond lengths of the ${ }^{1} E T_{d}$ state and ${ }^{1} A_{1} D_{2 d}$ state are shown in Table 3. The main difference in geometries between these two states is the shortening of eight bonds between inner $\left(\mathrm{Ti}^{\mathrm{i}}\right)$ and outer $\left(\mathrm{Ti}^{\mathrm{o}}\right)$ Ti atoms by $0.014 \AA$ and the elongating of the other four $\mathrm{Ti}^{\mathrm{i}}-\mathrm{Ti}^{\mathrm{o}}$ bonds by $0.019 \AA$ in the 
distorted structure. The other bond lengths are changed by less than $0.010 \AA$ as a result of the distortion.

The last column of Table 1 presents the relative energies calculated with multireference perturbation theory MRMP2 ${ }^{21}$. Although the MRMP2 trends are similar to those described above for MRCI(SD), the actual energies are rather different. Since MRMP2 calculations are generally much more computationally efficient than are MRCI(SD) calculations, it is important to explore the origin of the differences observed here. Analysis of the MRMP2 wave function reveals that the contribution from the zero-order CASSCF wave function to the total MRMP2 wave function is only $30 \%$. In other words the first order correction accounts for $70 \%$ of the MRMP2 wave function. This means the perturbation is not small here, and suggests the presence of intruder states. Therefore, the MRMP2 relative energies in Table 1 are not likely to be reliable.

Calculations using another version of multireference perturbation theory, CASPT2, ${ }^{22}$ confirm the presence of two types of intruder states. The first type corresponds to single excitations from several doubly occupied orbitals lying lower in energy than the CASSCF active space into active space orbitals. The second type of intruder states corresponds to single excitations from the active space to low lying external orbitals. One way to avoid these intruder states is to include additional doubly occupied and external orbitals in the active space.

The foregoing results suggest that a reasonable approach to the problem of strong electron correlation in $\mathrm{Ti}_{8} \mathrm{C}_{12}$ would be to increase the size of CASSCF active space, followed by MRMP2 to account for the dynamic part of electron correlation (since MRCI would be prohibitively expensive with a large CASSCF active space). A reasonable first step would be to increase the CASSCF active space in a way that both the $\left(7 \mathrm{t}_{2}\right)$ and $\left(4 \mathrm{t}_{1}\right) \mathrm{HF}$ determinants are included in the CASSCF wave function. Such an active space would contain four electrons in seven orbitals $\left(4 \mathrm{a}_{1}, 7 \mathrm{t}_{2}, 4 \mathrm{t}_{1}\right)$. However, it appears to be impossible to converge CASCSF to a wave function that has any significant contribution from both $\left(7 \mathrm{t}_{2}\right)$ and $\left(4 t_{1}\right)$ determinants, because the $7 t_{2}$ and $4 t_{1}$ orbitals are localized on different sets of $\mathrm{Ti}$ atoms. The $7 \mathrm{t}_{2}$ orbitals are centered on four $\mathrm{Ti}$ atoms on the inner tetrahedron, whereas the $4 t_{1}$ orbitals are centered on the $\mathrm{Ti}$ atoms of the capping (outer) tetrahedron. So, the 
Hamiltonian matrix elements between the $\left(7 t_{2}\right)$ and $\left(4 t_{1}\right)$ determinants are close to zero. Adding more occupied orbitals to the active space can solve this problem. The occupied orbitals ( $4 \mathrm{e}, 6 \mathrm{t}_{2}$ and $3 \mathrm{t}_{1}$ ) have dominant contributions from the dorbitals of all $8 \mathrm{Ti}$ atoms (Fig. 5) and thereby produce mixing between the ( $\left.7 \mathrm{t}_{2}\right)$ and ( $\left.4 \mathrm{t}_{1}\right)$ determinants in the CASSCF wave function. This suggests that correlation of all $20 \mathrm{Ti}$ valence electrons is necessary to get a balanced CASSCF active space.

The orbital energy diagram of Lin and Hall (Fig. 2) can be used to select the additional unoccupied orbitals. This results in an active space with 20 electrons in 24 orbitals. This active space should also solve the MRMP2 intruder states problem. However, an active space with 20 electrons in 24 orbitals is beyond the capability of any direct CASSCF code. Fortunately, one way to deal with such large active spaces is to use the ORMAS (occupation restricted multiple active space $)^{23}$ method. The three active spaces are illustrated in Figure 4. The first active space contains eight orbitals ( $4 \mathrm{e}, 6 \mathrm{t}_{2}$ and $3 \mathrm{t}_{1}$ ) and from 16 to 14 electrons (up to 2 holes). The second active space is equivalent to the original CASSCF active space ( $4 a_{1}$, $\left.7 t_{2}\right)$ and can be occupied by $2-6$ electrons. The third space contains 12 orbitals $\left(4 t_{1}, 5 a_{1}, 8 t_{2}\right.$, $\left.9 \mathrm{t}_{2}, 4 \mathrm{e}\right)$ and $0-2$ electrons. This particular choice of active spaces makes ORMAS equivalent to the restricted active space self-consistent field (RASSCF $)^{24}$ method, and potentially able to generate a wave function for any electronic state of interest.

The ORMAS natural orbitals and their occupation numbers for the $T_{d}{ }^{1} E$ state are shown in Figure 6 . The $4 a_{1}$ and $7 t_{2}$ occupation numbers differ significantly from zero and one, indicating the presence of strong electron correlation in this active space. In contrast, the occupation numbers of the orbitals in the first and third active spaces are close to two and zero respectively. However, it appearss to be necessary to include all 24 orbitals and 20 electrons in the active space in order to prevent the appearance of intruder states in multireference perturbation theory.

\section{Single reference calculations}

The results of single reference calculations are summarized in Table 4. It is clear that HF gives qualitatively wrong results, predicting the $T_{d}{ }^{5} A_{2}$ state to be the ground state. The $\mathrm{HF}$ electronic states that arise from distorted $\mathrm{D}_{2 \mathrm{~d}}$ and $\mathrm{C}_{3 \mathrm{v}}$ structures are predicted to be 50 - 
$100 \mathrm{kcal} / \mathrm{mol}$ higher in energy than ${ }^{5} \mathrm{~A}_{2}$. On the other hand, MP2 energies of the distorted structures are about $100 \mathrm{kcal} / \mathrm{mol}$ lower in energy than the ${ }^{5} \mathrm{~A}_{2} \mathrm{~T}_{\mathrm{d}}$ state. CCSD/MP2 predicts the ${ }^{5} \mathrm{~A}_{2}$ state to be the lowest in energy, but only by $19.5 \mathrm{kcal} / \mathrm{mol}$ relative to the $\mathrm{D}_{2 \mathrm{~d}}{ }^{1} \mathrm{~A}_{1}$ state ( $7 \mathrm{t}_{2}$ reference). $\mathrm{CCSD}(\mathrm{T}) / \mathrm{MP} 2$ predicts the states of the distorted structures to be about 50 $\mathrm{kcal} / \mathrm{mol}$ lower in energy than the $\mathrm{T}_{\mathrm{d}}{ }^{5} \mathrm{~A}_{2}$ state. There are significant differences between the B3LYP and CCSD(T) relative energies, although the B3LYP energies are closer to those of $\operatorname{CCSD}(\mathrm{T})$ than any other method. However, this is misleading, since, in contrast to HF and CASSCF, the B3LYP Hessians for $D_{2 d}$ and $C_{3 v}$ structures have several imaginary frequencies. This indicates that B3LYP favors even lower symmetry structures. This is in agreement with previously reported DFT calculations on $\mathrm{Ti}_{8} \mathrm{C}_{12 .}{ }^{4-7}$ Similar artificial distortions, caused by inadequate treatment of electron correlation by DFT, were observed in $\mathrm{C}_{36}$ fullerene $\mathrm{e}^{25}$.

The large differences among the MP2, CCSD and $\operatorname{CCSD}(T)$ energies indicates the presence of strong electron correlation in $\mathrm{Ti}_{8} \mathrm{C}_{12}$. This means, as noted earlier, that the $\mathrm{HF}$ wave function is a poor zeroth-order approximation. To obtain accurate energies for systems like $\mathrm{Ti}_{8} \mathrm{C}_{12}$, within the framework of single reference methods, it is necessary to use a relatively high order of excitation (beyond singles and doubles). It is well known that perturbation theory can diverge as the excitation level (perturbation order) increases ${ }^{26}$. One test of the reliability of single reference methods may be calculations of the energies of a given state, based on different HF reference wave functions (solutions). Since at the full configuration interaction (FCI) limit the energy does not depend on the choice of HF reference, a "good" post-HF method should produce $\sim$ the same energies for these two solutions. Figure 6 shows the energy difference between two HF solutions $\left({ }^{1} A_{1}\left(7 t_{2}\right)\right.$ and ${ }^{1} A_{1}\left(4 t_{1}\right)$ ) for the $D_{2 d}{ }^{1} A_{1}$ state, calculated with perturbation theory and coupled cluster methods. The energies of both solutions were calculated at the MP2 optimized geometry of the ${ }^{1} \mathrm{~A}_{1}\left(7 \mathrm{t}_{2}\right)$ solution. Examination of the MP2, MP3 and MP4 energies makes it clear that the perturbation series diverges. The MP4(SDTQ) energies of the two solutions differ by 118.7 $\mathrm{kcal} / \mathrm{mol}$, whereas, the MP2 energies differ by only $19.9 \mathrm{kcal} / \mathrm{mol}$. In contrast, the CCSD and $\operatorname{CCSD}(\mathrm{T})$ energy differences are 16.2 and $1.5 \mathrm{kcal} / \mathrm{mol}$, respectively. This suggests that the $\operatorname{CCSD}(\mathrm{T})$ level of theory, which includes up to triple excitations, is able to account for most 
of the correlation energy. However, simply including the triple excitations is not sufficient, since MP4 includes triple excitations perturbatively, as does $\operatorname{CCSD}(\mathrm{T})$. The difference is that in the CC ansatz, higher order excitations (clusters) are included to some degree via products of singles and doubles (i.e., unlinked clusters). This makes $\mathrm{CC}$ theory more robust than perturbation theoy which includes no such higher order terms. Since neither perturbation theory nor coupled cluster theory are variational methods, the lowest energy solution does not necessarily correspond to the more accurate representation of the ${ }^{1} A_{1}$ electronic state. However, the $\operatorname{CCSD}(T)$ energy difference between the two solutions for ${ }^{1} A_{1}$ is very small. This provides some confidence that $\operatorname{CCSD}(\mathrm{T})$ is able to provide accurate results for this highly multireference system. An alternative view is provided by the $T_{1}$ diagnostic, which is often used as an indicator of possible problems in a coupled cluster calculation due to multireference character of the wave function ${ }^{27}$. The values of the $\mathrm{T}_{1}$ diagnostic for most of the states of $\mathrm{Ti}_{8} \mathrm{C}_{12}$ is about 0.05 , which is usually considered to be the threshold for usefulness of $\operatorname{CCSD}(\mathrm{T})$ results.

In order to justify correlating only 56 electrons in the $\mathrm{MRCI}(\mathrm{SD})$ calculations the MP2, CCSD and CCSD(T) calculations were re-evaluated using 20 and 56 correlated electrons, as well as the full 80 valence electrons. The results, presented in Table 5, clearly show that correlating only 20 electrons is not sufficient at any of these levels of theory. On the other hand, reasonably accurate relative energies can be obtained by correlating only 56 electrons. The quality of the 56-electron results is clearly method-dependent. There are still large $(\sim 7.12 \mathrm{kcal} / \mathrm{mol})$ errors for MP2, whereas for $\operatorname{CCSD}(\mathrm{T})$ the largest error in relative energies introduced by correlating 56 , rather than all 80 , valence electrons is only 3.1 $\mathrm{kcal} / \mathrm{mol}$.

\section{Calculation of ionization potential}

While there is relatively little quantitative experimental data available for $\mathrm{Ti}_{8} \mathrm{C}_{12}$, accurate experimental values for the ionization potential and electron affinity are known. Calculation of electron affinities requires large basis sets that include diffuse functions. This would extend the most important levels of theory used here beyond current computational capabilities. On the other hand, the accurate prediction of the ionization potential should be 
possible even with the 6-31G(d) basis set. In Table 6 the vertical and adiabatic (for some methods) ionization potentials (IP) of $\mathrm{D}_{2 \mathrm{~d}} \mathrm{Ti}_{8} \mathrm{C}_{12}$ are reported. The IP was calculated as an energy difference between the ${ }^{\mathrm{I}} \mathrm{A}_{1}\left(7 \mathrm{t}_{2}\right)$ state of $\mathrm{Ti}_{8} \mathrm{C}_{12}$ and the ${ }^{2} \mathrm{~B}_{1}\left(7 \mathrm{t}_{2}\right)$ state of $\mathrm{Ti}_{8} \mathrm{C}_{12}{ }^{4}$. The $\mathrm{HF}$ method predicts a very large vertical IP of $7.09 \mathrm{eV}$. This is not surprising since the $\mathrm{HF}$ method predicts the $T_{d}{ }^{5} A_{2}$ state to be the ground state. MP2 (CCSD) overestimates (underestimates) the experimental IP $(4.40 \pm 0.02 \mathrm{eV})^{28}$ by $1.11(0.75) \mathrm{eV}$. CCSD(T) predicts a vertical IP of $4.10 \mathrm{eV}$, which it is still $0.30 \mathrm{eV}$ below the experimental value. It is very likely that the remaining disparity for $\operatorname{CSSD}(\mathrm{T})$ can be attributed to basis set deficiencies. So, an $0.30 \mathrm{eV}$ error with a small basis set is reasonable. The CASSCF method, which correlates only 4 electrons in 4 orbitals, improves the $\mathrm{HF}$ result by $0.80 \mathrm{eV}$. MRCI(SD) overestimates the IP by $0.85 \mathrm{eV}$. However, including the Davidson correction for quadruple excitations $(\mathrm{MRCl}(\mathrm{SD})+\mathrm{Q}$ ) predicts an IP of $4.73 \mathrm{eV}$, only $0.33 \mathrm{eV}$ above the experimental value. Again, one expects this prediction to improve with a better basis set. Interestingly, DFT/ B3LYP predicts an IP of $4.03 \mathrm{eV}$, only $0.37 \mathrm{eV}$ below the experimental value.

\section{Conclusions}

The distortion of $T_{d} T_{8} C_{12}$ to $D_{2 d}$ and $C_{3 v}$ (near $T_{d}$ ) geometries due to the Jahn-Teller effect was confirmed using the CASSCF MRCI method. The highest level of single reference calculations reported in this paper $(\mathrm{CCSD}(\mathrm{T})$ energies at MP2 optimized geometry with 6$31 \mathrm{G}(\mathrm{d})$ basis set) predict the ${ }^{1} \mathrm{~A}_{1}$ and ${ }^{3} \mathrm{~B}_{1}$ states of distorted $D_{2 d}$ are the lowest energy states. These two states can be considered to be quasidegenerate with an energy difference of less than $3 \mathrm{kcal} / \mathrm{mol}$. However, the $\mathrm{C}_{3 \mathrm{v}}{ }^{3} \mathrm{~A}_{2}$ state might be lower in energy than the $\mathrm{D}_{2 \mathrm{~d}}{ }^{1} \mathrm{~A}_{1}$ and ${ }^{3} \mathrm{~B}_{1}$ states. It is likely that MRCI(SD) and $\operatorname{CCSD}(\mathrm{T})$ are adequate levels of theory for this system, but larger basis sets are clearly needed, and $\mathrm{MRCI}$ needs to correlate all 80 valence electrons. The $\mathrm{Ti}_{8} \mathrm{C}_{12} \mathrm{D}_{2 \mathrm{~d}}$ ionization potential, calculated with $\mathrm{MRCI}(\mathrm{SD})+\mathrm{Q}(4.73 \mathrm{eV})$ and $\operatorname{CCSD}(\mathrm{T})(4.10 \mathrm{eV})$ are in reasonable agreement with the experimental value of $4.40 \pm 0.02$ $\mathrm{eV}$.

The results presented here demonstrate that both nondynamic and dynamic parts of electron correlation have to be treated well in order to accurately describe the electronic 
structure of $\mathrm{Ti}_{8} \mathrm{C}_{12}$. The ORMAS active space with 20 electrons and 24 orbitals was used to obtain zero-order wave function for future large-scale MRMP2 calculations. In some cases correlating all 80 valence electrons of $\mathrm{Ti}_{8} \mathrm{C}_{12}$ is probably unnecessary, however, as previously reported, it is not enough to correlate only 20 electrons. As an example it is shown that the largest error of $\operatorname{CCSD}(\mathrm{T})$ calculations with 56 correlated electrons is only $3.1 \mathrm{kcal} / \mathrm{mol}$.

\section{Acknowledgments}

This work was supported by a grant from the Air Force Office of Scientific Research. Computer time was made available at the NERSC center for some of the larger calculations. The calculations in this work were performed in part on an IBM workstation cluster made possible by grants from IBM in the form of a Shared University Research grant and the United States Department of Energy. The authors thank Dr. Michael Schmidt for helpful discussions.

\section{References}

${ }^{1}$ B.C. Guo, K.P. Kerns, and A.W. Castleman, Jr., Science 255, 1411 (1992); B.C. Guo, S. Wei, J. Purnell, S.A. Buzza, and A.W. Castleman, Jr., Science 256, 515 (1992).

${ }^{2}$ M.-M. Rohmer, M. Bénard, J.-M. Poblet, Chem. Rev. 100, 495, (2000).

${ }^{3}$ I. Dance, J. Chem. Soc., Chem. Commun. 1779, (1992); I. Dance, J. Am. Chem. Soc. 118 , 6309 (1996).

${ }^{4}$ G.K. Gueorguiev, J.M. Pacheco, Phys. Rev. Lett. 88, 115504, (2002).

${ }^{5}$ T. Baruah, M.R. Pederson, M.L. Lyn and A.W. Castleman, Jr. Phys. Rev. A, 66, 053201 (2002).

${ }^{6}$ K. Deng, W. Duan, B. Gu, J. Chem. Phys. 121, 4123, (2004).

${ }^{7}$ H. Hou, J.T. Muckerman, P. Liu, J.A. Rodriguez, J. Phys. Chem. A, 107, 9344, (2003).

${ }^{8}$ B.O. Roos, Adv. Chem. Phys. 69, 399, (1987); J. Ivanic, K. Ruedenberg, Theoret. Chem. Acc. 106, 339, (2001). 
${ }^{9}$ H.-J. Werner and P.J. Knowles, J. Chem. Phys. 89, 5803 (1988); P.J. Knowles and H.-J. Werner, Chem. Phys. Lett. 145, 514 (1988).

${ }^{10}$ R.J. Bartlett, J. Phys. Chem. 93, 1697 (1989); C. Hampel, K. Peterson, and H.-J. Werner, Chem. Phys. Lett. 190, 1 (1992) and references therein. The program to compute the perturbative triples corrections has been developed by M.J.O. Deegan and P.J. Knowles (1992).

${ }^{11}$ C. Møller, M.S. Plesset, Phys. Rev. 46, 618 (1934); J.A. Pople, J.S. Binkley, R. Seeger Int. J. Quant. Chem. S10, 1, (1976); M.J. Frisch, M. Head-Gordon, J.A. Pople, Chem. Phys. Lett. 166, 275, (1990); G.D. Fletcher, M.W. Schmidt, M.S. Gordon, Adv. Chem. Phys. 110, 267, (1999); C.M. Aikens, S.P. Webb, R.L. Bell, G.D. Fletcher, M.W. Schmidt, M.S. Gordon, Theor. Chem. Acc. 110, 233, (2003); C.M. Aikens, M.S. Gordon J. Phys. Chem. A, 108, (2004).

${ }^{12}$ T.J. Lee, D. Jayatilaka, Chem. Phys. Lett. 201, 1 (1993); T.J. Lee, A.P. Rendell, K.G. Dyall, D. Jayatilaka, J. Chem. Phys. 100, 7400 (1994).

${ }^{13}$ P.J. Knowles, C. Hampel, and H.-J. Werner, J. Chem. Phys. 99, 5219 (1993).

${ }^{14}$ A.D. Becke, J. Chem. Phys. 98, 5648 (1993); P.J. Stephens, F.J. Devlin, C.F. Chablowski, M.J. Frisch, J. Phys. Chem. 98, 11623 (1994); R.H. Hertwig, W. Koch, Chem. Phys. Lett. 268,345 (1997).

${ }^{15}$ W.J. Hehre, R. Ditchfield and J.A. Pople, J. Chem. Phys. 56, 2257 (1972); V.A. Rassolov, J.A. Pople, M.A. Ratner, T.L. Windus, J. Chem. Phys. 109, 1223, (1998).

${ }^{16}$ M.W. Schmidt, K.K. Baldridge, J. A. Boatz, S.T. Elbert, M.S. Gordon, J.H. Jensen, S. Koseki, N. Matsunaga, K.A. Nguyen, S.J. Su, T.L. Windus, M. Dupuis, J.A. Montgomery, J. Comp.Chem. 14, 1347 (1993); G.D. Fletcher, M.W. Schmidt, M.S. Gordon, Adv. Chem. Phys. 110, 267 (1999); G.D. Fletcher, M.W. Schmidt, B.M. Bode, M.S. Gordon, Comput. Phys. Commun. 128, 190 (2000).

${ }^{17}$ MOLPRO is a package of ab-initio programs written by H.-J. Werner and P.J. Knowles, with contributions from R.D. Amos, A. Bernhardsson, A. Berning, P. Celani, D.L. Cooper, M.J.O. Deegan, A.J. Dobbyn, F. Eckert, C. Hampel, G. Hetzer, T. Korona, R. Lindh, A.W. Lloyd, S.J. McNicholas, F.R. Mandy, W. Meyer, M.E. Mura, A. Nicklass, P. Palmieri, R. Pitzer, G. Rauhut, M. Schutz, H. Stoll, A.J. Stone, R. Tarroni, and T. Thorsteinsson. 
${ }^{18}$ Z.X. Lin, M.B. Hall, J. Am. Chem. Soc. 115, 11165, (1993).

${ }^{19}$ M. Bénard, M.-M. Rohmer, J.-M. Poblet, C. Bo, J. Phys. Chem. 99, 16913, (1995).

${ }^{20}$ M.-M. Rohmer, M. Bénard, C. Henriet, C. Bo, J.-M. Poblet, J. Chem. Soc., Chem. Commun. 1182, (1993).

${ }^{21}$ K. Hirao, Chem. Phys. Lett. 190, 374, (1992); H. Nakano, J. Chem. Phys. 99, 7983 , (1993); H. Umeda, S. Koseki, U. Nagashima, M.W. Schmidt, J. Comput. Chem. 22, 1243, (2001);

${ }^{22}$ K. Andersson, P.-A. Malmqvist, B.O. Roos, J. Chem. Phys. 96, 1218 (1992); H.-J. Werner, Mol. Phys. 89, 645-661 (1996); P. Celani and H.-J. Werner, J. Chem. Phys. 112, 5546 $(2000)$.

${ }^{23}$ J. Ivanic J. Chem. Phys. 119, 9364, (2003); J. Ivanic J. Chem. Phys. 119, 9377, (2003).

${ }^{24}$ J. Olsen, B.O. Roos, P. Jrgensen, H.J.Aa. Jensen, J. Chem. Phys. 89, 2185 (1998).

${ }^{25}$ S.A. Varganov, P.V. Avramov, S.G. Ovchinnikov, M.S. Gordon, Chem. Phys. Lett. 362, $380,(2002)$.

${ }^{26}$ J. Olsen, O. Christiansen, H. Koch, P.J. Jørgensen, J. Chem. Phys. 105, 5082, (1996); T.H. Dunning Jr., K.A. Peterson, J. Chem. Phys. 108, 4761 (1998); B. Forsberg, Z. He, Y. He, D. Cremer, Int. J. Quantum Chem. 76, 306, (2000); M. L. Leininger, W.D. Allen, H.F. Schaefer III, C.D. Sherrill, J. Chem. Phys. 112, 9213, (2000).

${ }^{27}$ T.J. Lee and P.R. Taylor, Int. J. Quant. Chem. S23, 199 (1989).

${ }^{28}$ H. Sakurai, A.W. Castleman, Jr. J. Phys. Chem. A, 102, 10486, (1998). 
Table 1. The relative energies $(\mathrm{kcal} / \mathrm{mol})$ of lowest energy states of $T_{c 1}$ structure and ' $A_{1}$ state of $D_{2 d}$ structure. CASSCF active space consists of four electrons on $4 a_{1}$ and $7 t_{2}$ orbitals.

\begin{tabular}{|c|c|c|c|c|c|c|}
\hline & CASSCF & $\operatorname{MRCl}(20 \mathrm{e})$ & $\mathrm{MRCI}+\mathrm{Q}(20 \mathrm{e})$ & MRCI(56e) & $\mathrm{MRCI}+\mathrm{Q}(56 \mathrm{c})$ & MRMP2 \\
\hline \multicolumn{7}{|l|}{$\overline{T_{d} \text { structure }}$} \\
\hline${ }^{5} \mathrm{~A}_{2}$ & 0.0 & 0.0 & 0.0 & 0.0 & 0.0 & 0.0 \\
\hline${ }^{3} \mathrm{~T}_{1}$ & -5.7 & -9.5 & -11.5 & -8.6 & -10.4 & -29.9 \\
\hline${ }^{\prime} \mathrm{E}$ & -8.7 & -14.4 & $-17,3$ & -13.0 & -15.5 & -44.3 \\
\hline \multicolumn{7}{|l|}{$\mathrm{D}_{x \Delta}$ structure } \\
\hline${ }^{3} \mathrm{~A}_{2}$ & -6.1 & -10.3 & -12.3 & -9.4 & -11.2 & -35.2 \\
\hline & -9.8 & -16.2 & -19.2 & -14.8 & -17.7 & -60.2 \\
\hline $\begin{array}{l}\mathrm{C}_{3} \text {, structure } \\
{ }^{3} \mathrm{~A}_{2}\end{array}$ & -6.9 & & & & & -44.6 \\
\hline
\end{tabular}


Table 2. The CASSCF natural orbital occupation numbers of $T_{d}$ structure and distorted $D_{2 d}$ and $\mathrm{C}_{3 \mathrm{v}}$ structures.

\begin{tabular}{|c|c|c|c|c|c|c|c|c|}
\hline \multicolumn{4}{|c|}{$T_{d}$ structure } & \multicolumn{3}{|c|}{$\overline{D_{t \alpha}}$ strucure } & \multicolumn{2}{|c|}{$\overline{\mathrm{C}_{\mathrm{gr}} \text { structure }}$} \\
\hline Orbital & ${ }^{3} A_{2}$ & $T_{1}$ & $\mathrm{E}$ & Orbital & ${ }^{\top} A_{1}$ & ${ }^{3} A_{2}$ & Orbital & ${ }^{3} A_{2}$ \\
\hline $4 a_{5}$ & 1.000 & 1.272 & $1.40 \mathrm{I}$ & $a_{;}$ & 1.441 & 1.289 & $a_{1}$ & 1.379 \\
\hline $7 t_{2}$ & $\begin{array}{l}1.000 \\
1.000 \\
1.000\end{array}$ & $\begin{array}{l}0.909 \\
0.909 \\
0.909\end{array}$ & $\begin{array}{l}0.866 \\
0.866 \\
0.866 \\
\end{array}$ & $\begin{array}{l}b_{1} \\
e \\
e\end{array}$ & $\begin{array}{l}1.199 \\
0.680 \\
0.680\end{array}$ & $\begin{array}{l}0.711 \\
1.000 \\
1.000\end{array}$ & $\begin{array}{l}a_{1} \\
\mathrm{e} \\
\mathrm{e}\end{array}$ & $\begin{array}{l}0.621 \\
1.000 \\
1.000\end{array}$ \\
\hline
\end{tabular}


Table 3. The CASSCF optimized bond distances $(\AA)$ for different electronic states of $\mathrm{Ti}_{8} \mathrm{C}_{12}$. The numbers in square brackets indicate the numbers of equivalent bonds.

\begin{tabular}{|c|c|c|c|c|c|c|}
\hline & \multicolumn{3}{|c|}{$T_{\mathrm{d}}$ structure } & \multicolumn{2}{|c|}{$\mathrm{D}_{2 \mathrm{~d}}$ strucure } & \multirow{2}{*}{$\frac{\mathrm{C}_{3 \mathrm{r}} \text { structure }}{{ }^{3} \mathrm{~A}_{1}}$} \\
\hline & ${ }^{3} A_{2}$ & ${ }^{3} T_{1}$ & ${ }^{3} \mathrm{E}$ & ${ }^{3} \mathrm{~A}_{2}$ & ${ }^{\prime} A_{1}$ & \\
\hline \multirow[t]{3}{*}{$\overline{T^{i}-T^{i}}$} & 2.935 [12] & $2.926[12]$ & $2.922[12]$ & $2.929[8]$ & $2.908[8]$ & 2.926 [3] \\
\hline & & & & $2.917[4]$ & $2.941[4]$ & 3.038 [3] \\
\hline & & & & & & $2.929[6]$ \\
\hline \multirow[t]{2}{*}{$\mathrm{C}-\mathrm{C}$} & $1.328[6]$ & $1.327[6]$ & $1.327[6]$ & 1.327 [4] & $1.326[4]$ & $1.326[3]$ \\
\hline & & & & $1.327[2]$ & $1.328[2]$ & $1.328[3]$ \\
\hline \multirow[t]{3}{*}{$\mathrm{Ti}^{\mathrm{n}}-\mathrm{C}$} & 1.954 [12] & $1.954[12]$ & $1.955[12]$ & $1.954[4]$ & $1.955[4]$ & 1.955 [3] \\
\hline & & & & $1.955[8]$ & $1.955[8]$ & $1.955[3]$ \\
\hline & & & & & & $1.954[6]$ \\
\hline \multirow[t]{4}{*}{$\mathrm{Ti}^{\mathrm{i}}-\mathrm{C}$} & $2.250[24]$ & $2.246[24]$ & $2.245[24]$ & 2.247 [8] & $2.239[8]$ & $2.248[6]$ \\
\hline & & & & $2.248[8]$ & $2.240[8]$ & 2.253 [6] \\
\hline & & & & $2.243[8]$ & $2.252[8]$ & $2.245[6]$ \\
\hline & & & & & & $2.236[6]$ \\
\hline
\end{tabular}


Table 4. The relative energies ( $\mathrm{kcal} / \mathrm{mol})$ of different states of $\mathrm{Ti}_{8} \mathrm{C}_{12}$ calculated with single reference methods. The XXX//YYY notation means that XXX method was used to calculate energy at $\mathrm{Ti}_{8} \mathrm{C}_{12}$ geometry optimized with $\mathrm{YYY}$ method.

\begin{tabular}{|c|c|c|c|c|c|c|c|c|}
\hline & $\mathrm{HF}$ & $\mathrm{MP} 2 / \mathrm{HF}$ & $\mathrm{CCSD} / \mathrm{HF}$ & $\mathrm{CCSD}(\mathrm{T}) / \mathrm{HF}$ & $\mathrm{MP} 2 / \mathrm{MP} 2$ & $\mathrm{CCSD} / / \mathrm{MP} 2$ & $\operatorname{CcSD}(\mathrm{T}) / \mathrm{MP} 2$ & B3LYP \\
\hline \multicolumn{9}{|l|}{$T_{d}$ structure } \\
\hline${ }^{s} A_{2}\left(4 a_{1} 7 t_{2}\right)$ & 0.0 & 0.0 & 0.0 & 0.0 & 0.0 & 0.0 & 0.0 & 0.0 \\
\hline${ }^{s} A_{:}\left(4 a_{1} 4 t_{1}\right)$ & 145.8 & 49.4 & & & -56.6 & & & 31.9 \\
\hline${ }^{\prime} A_{4}\left(5 a_{1}\right)$ & 126.1 & -104.5 & 31.4 & -13.5 & -105.8 & 28.9 & -29.2 & 11.7 \\
\hline \multicolumn{9}{|l|}{$D_{2 s}$ structure } \\
\hline${ }^{1} A_{1}\left(71_{2}\right)$ & 100.7 & -119.5 & 1.8 & 42.8 & -152.7 & 19.5 & -52.9 & -13.6 \\
\hline${ }^{1} A_{1}\left(4 t_{1}\right)$ & 104.7 & -119.6 & 11.2 & -49.3 & -135.3 & 32.4 & -55.2 & -12.6 \\
\hline${ }^{3} A_{2}\left(7 t_{3}\right)$ & 78.6 & -103.9 & -4.4 & -41.5 & & & & \\
\hline${ }^{3} A_{2}\left(4 t_{1}\right)$ & 90.2 & -97.2 & & & & & & \\
\hline${ }^{3} B_{1}\left(7 t_{2} 4 t_{2}\right)$ & 100.1 & -130.0 & 52 & -51.7 & -145.2 & 25.9 & -53.1 & -17.8 \\
\hline \multicolumn{9}{|l|}{$\mathrm{C}_{3 r}$ structure } \\
\hline${ }^{1} A_{1}\left(7 t_{2}\right)$ & 88.0 & -66.2 & 7.5 & -39.1 & & & & -10.5 \\
\hline${ }^{\prime} A_{1}\left(4 t_{1}\right)$ & 97.3 & -105.6 & 8.5 & -44.9 & & & & -7.5 \\
\hline${ }^{3} A_{2}\left(7 t_{2}\right)$ & 50.7 & -75.1 & & & & & & -19.2 \\
\hline${ }^{3} \mathrm{~A}_{1}\left(7 \mathrm{t}_{2} 8 \mathrm{t}_{2}\right)$ & 30.4 & -42.4 & & & & & & no conv." \\
\hline
\end{tabular}

${ }^{a}$ The iterative method used to solved Kohn-Sham equation did not converge 
Table 5. The relative energies $(\mathrm{kcal} / \mathrm{mol})$ of different states of $\mathrm{Ti}_{8} \mathrm{C}_{12}$ calculated with different number of correlated electrons.

\begin{tabular}{|c|c|c|c|c|c|c|c|c|c|c|}
\hline & \multirow[t]{2}{*}{$\mathrm{HF}$} & \multicolumn{3}{|c|}{$\mathrm{MP2}$} & \multicolumn{3}{|c|}{ CCSD } & \multicolumn{3}{|c|}{$\operatorname{CcsD}(\mathrm{T})$} \\
\hline & & $20 \mathrm{e}$ & $56 \mathrm{e}$ & $80 \mathrm{e}$ & $20 \mathrm{c}$ & $56 \mathrm{e}$ & $80 \mathrm{e}$ & $20 \mathrm{e}$ & $56 \mathrm{e}$ & $80 \mathrm{e}$ \\
\hline \multicolumn{11}{|c|}{$T_{d}$ structure } \\
\hline${ }^{5} \mathrm{~A}_{2}\left(4 \mathrm{a}_{1} 7 \mathrm{t}_{2}\right)$ & 0.0 & 0.0 & 0.0 & 0.0 & 0.0 & 0.0 & 0.0 & 0.0 & 0.0 & 0.0 \\
\hline${ }^{1} A_{1}\left(5 a_{1}\right)$ & 124.6 & -15.8 & -98.3 & -105.9 & 56.6 & 32.1 & 28.9 & 12.9 & -26.1 & -29.2 \\
\hline \multicolumn{11}{|c|}{$D_{z e}$ structure } \\
\hline${ }^{1} A_{1}\left(7 \ell_{2}\right)$ & 134.8 & -27.0 & -139.5 & -152.6 & 26.6 & 21.7 & 19.5 & -37.2 & -51.2 & -52.9 \\
\hline${ }^{1} A_{1}\left(4 t_{L}\right)$ & 133.0 & 40.5 & -125.2 & -135.3 & 50.1 & 35.6 & 32.4 & -24.5 & -53.0 & -55.2 \\
\hline${ }^{3} \mathrm{~B}_{1}\left(7 \mathrm{t}_{2} 4 \mathrm{t}_{1}\right)$ & 128.8 & -50.8 & -133.8 & -145.1 & 39.2 & 28.2 & 25.9 & -29.1 & -52.8 & -53.1 \\
\hline
\end{tabular}


Table 6. The ionization potential (eV), calculated as an energy difference between ${ }^{1} A_{1}$ state of $\mathrm{Ti}_{8} \mathrm{C}_{12} \quad \mathrm{D}_{2 \mathrm{~d}}$ structure and ${ }^{2} \mathrm{~B}_{2}$ state of $\mathrm{Ti}_{8} \mathrm{C}_{12}{ }^{+} \mathrm{D}_{2 \mathrm{~d}}$ structure.

\begin{tabular}{lccccccccc}
\hline \hline & HF & MP2 $^{2}$ & CCSD $^{*}$ & CCSD (T) $^{\prime}$ & B3LYP & CASSCF $^{5}$ & MRCI $^{\circ}$ & MRCI+Q $^{*}$ & Exp $^{4}$ \\
\hline IP vertical & 7.07 & 5.51 & 3.65 & 4.10 & 4.03 & 6.28 & 5.35 & 4.73 & $4.40 \pm 0.02$ \\
IP adiabatic & 6.92 & & & & 3.93 & 6.00 & & & \\
\hline \hline
\end{tabular}

${ }^{a} \mathrm{MP2}$ optimized geometry.

${ }^{b}$ CASSCF active space consists of four electrons on four $\left(4 \mathrm{a}_{1}, 7 \mathrm{t}_{2}\right)$ orbitals.

"MRCI with 56 correlated electrons.

${ }^{d}$ Experimental value from Ref. 28. 

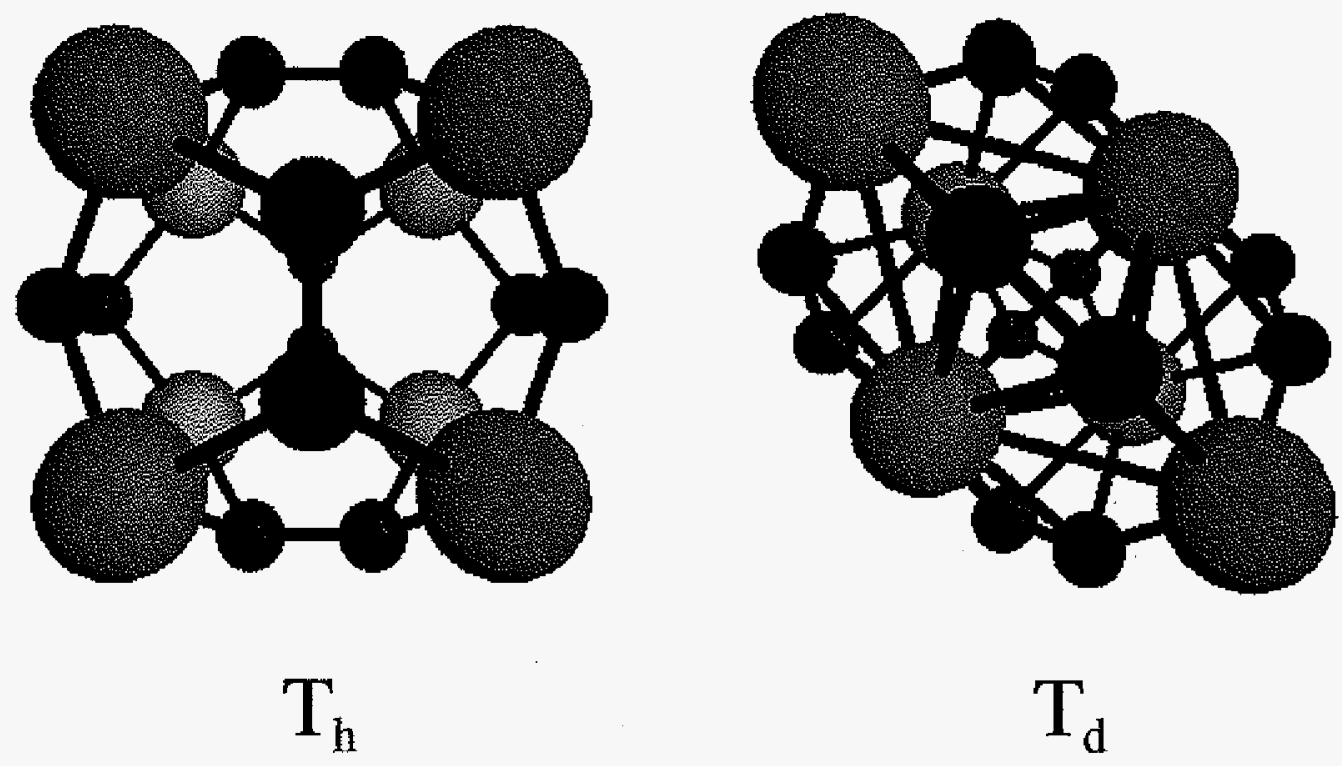

Figure 1. $T_{b}$ and $T_{d}$ structures of $\mathrm{Ti}_{8} \mathrm{C}_{12}$ Met-car. 


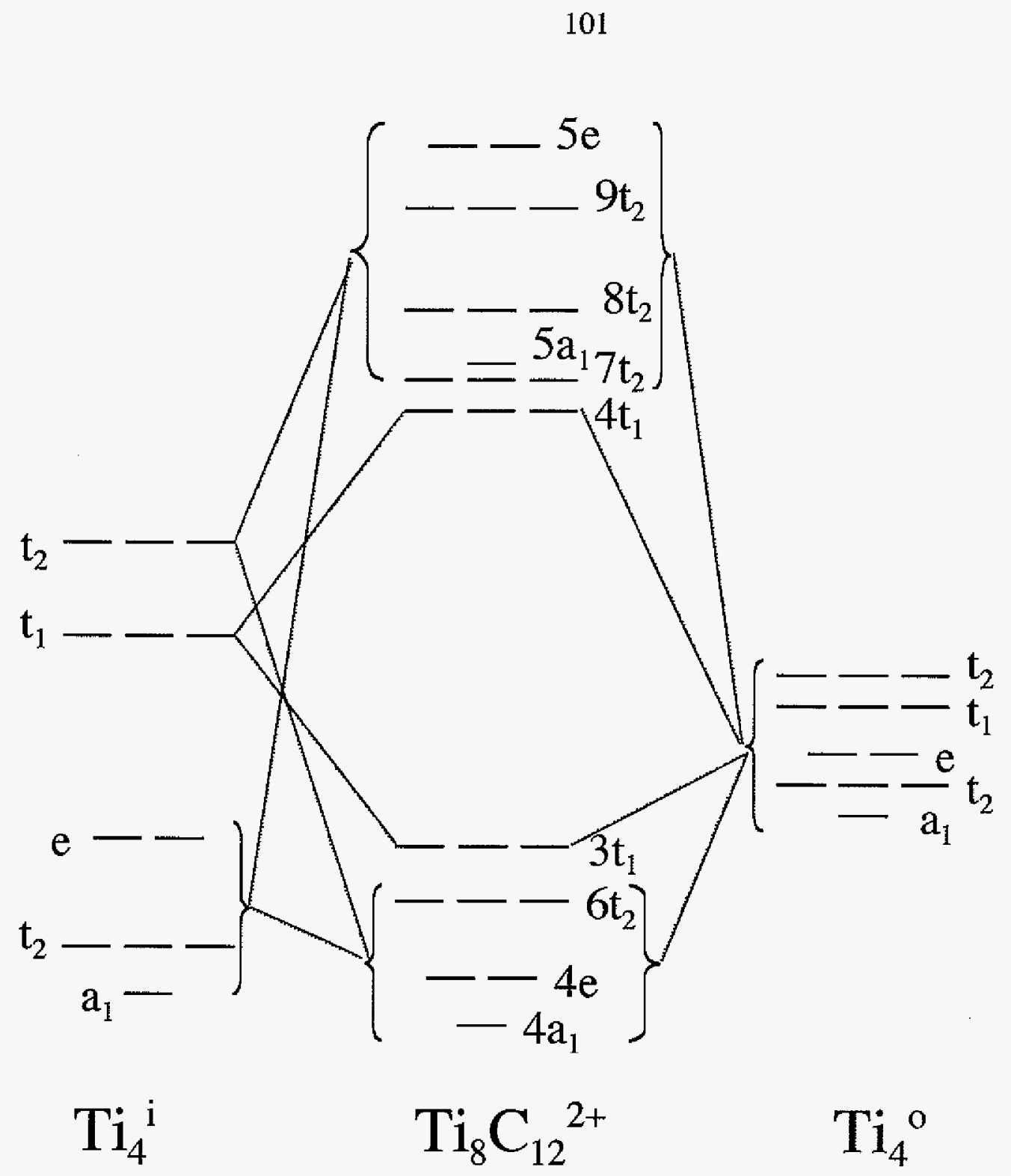

Figure 2. Orbital diagram of Lin and Hall [Ref. 19]. The $\mathrm{Ti}_{8} \mathrm{C}_{12}{ }^{2+}$ orbitals are produced by interaction of $\mathrm{Ti} \mathrm{d}_{\mathrm{z} 2}, \mathrm{~d}_{\mathrm{xz}}$ and $\mathrm{d}_{\mathrm{yz}}$ orbitals of inner $\left(\mathrm{Ti}_{4}{ }^{\mathrm{j}}\right)$ and outer $\left(\mathrm{Ti}_{4}{ }^{\circ}\right)$ tetrahedra. 


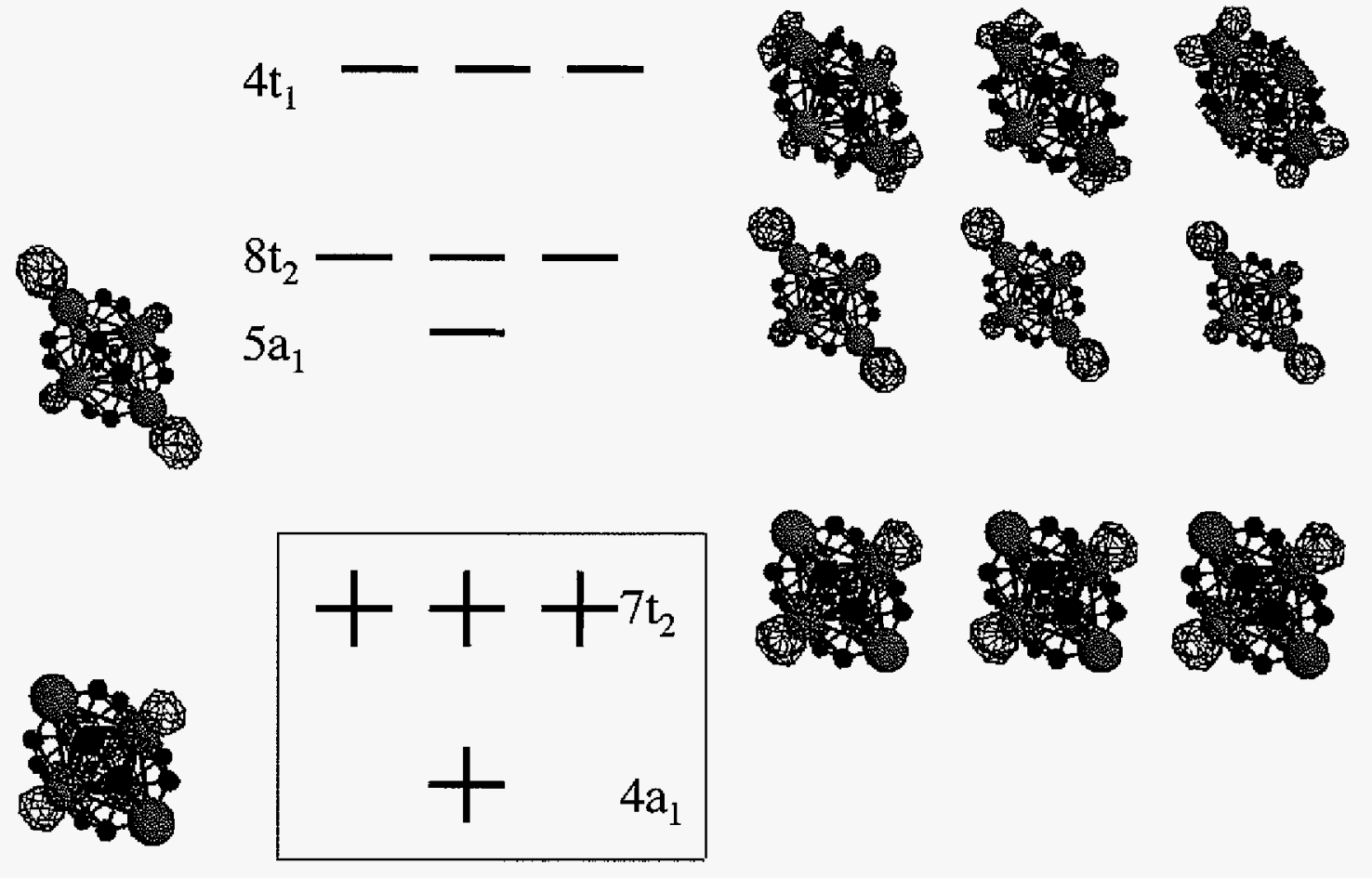

Figure 3. Several highest occupied and lowest inoccupied Hartree-Fock orbitals of $T_{d}$ structure of $\mathrm{Ti}_{8} \mathrm{C}_{12}$. The CASSCF active space is contained in the dashed box. 


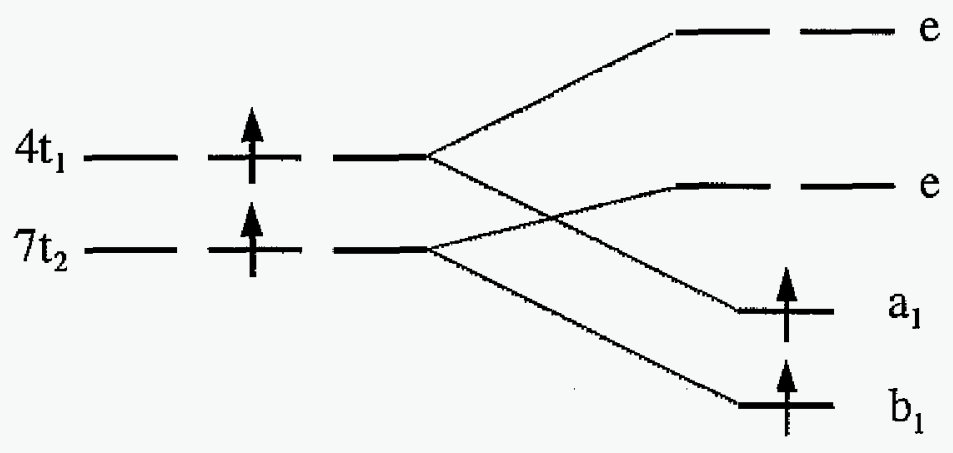

Figure 4. Orbital diagram of distortion of $\mathrm{T}_{\mathrm{d}}$ structure to $\mathrm{D}_{2 \mathrm{~d}}$ structure with ${ }^{3} \mathrm{~B}_{1}$ electronic state. 


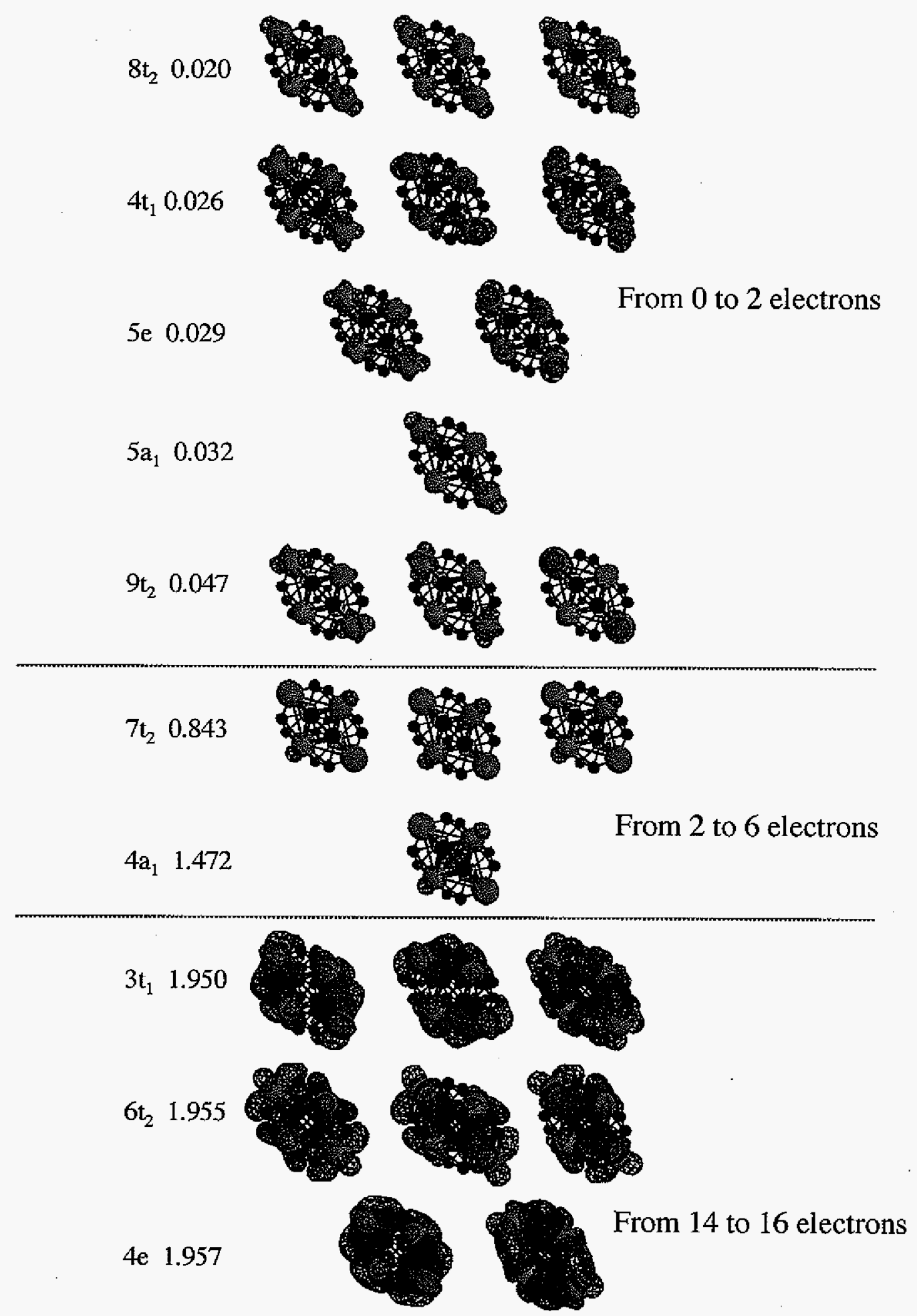

Figure 5. ORMAS optimized naturals orbitals and their occupation numbers for ${ }^{1} \mathrm{E}_{1}$ state of $T_{d}$ structure. The three active spaces are separated by dashed line. The minimal and maximal numbers of electrons in each active space are indicated. 


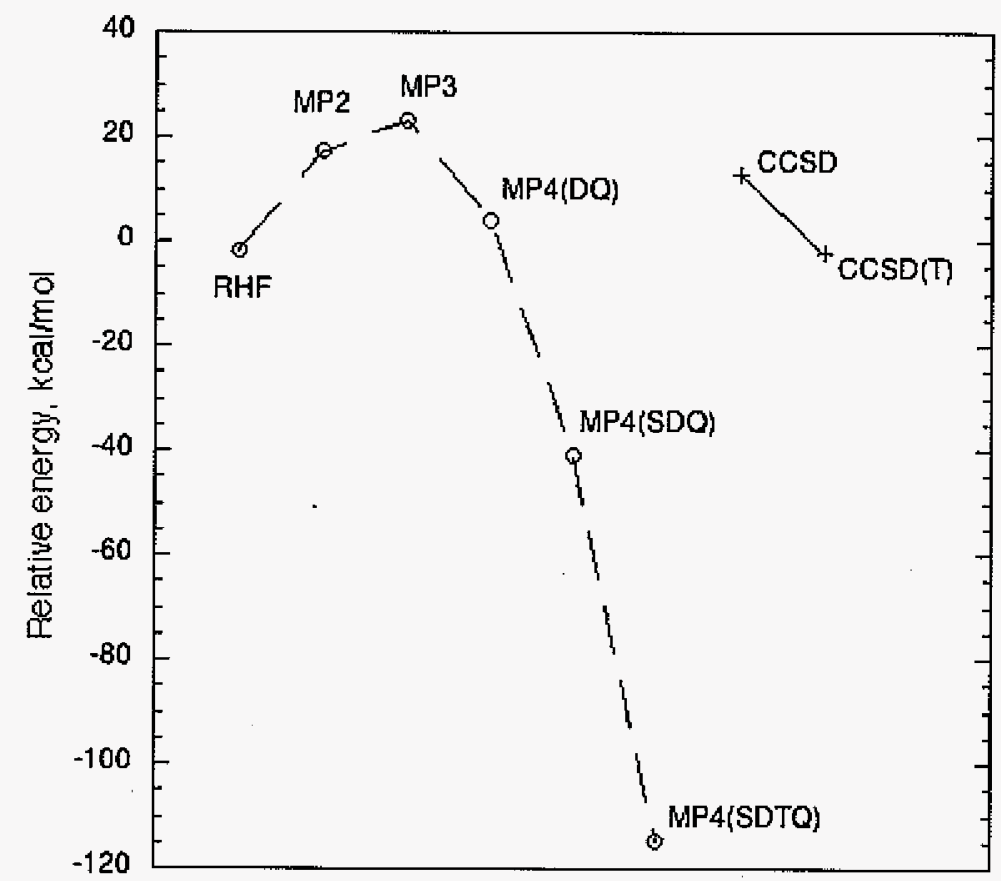

Figure 6. Relative energy of ${ }^{1} A_{1}\left(7 t_{2}\right)$ solution with respect to ${ }^{1} A_{1}\left(4 t_{1}\right)$ solution (kcal/mol) calculated with different single reference methods. The MP2 optimized geometry of ${ }^{1} A_{1}\left(7 t_{2}\right)$ solution is used for energies calculations of both solutions. 


\title{
CHAPTER 6. DENSITY CUMULANT FUNCTIONAL THEORY
}

Taken from a paper to be submitted to the Journal of Chemical Physics.

\author{
Sergey A. Varganov and Mark S. Gordon
}

\begin{abstract}
A new method for solving the stationary electronic Schrödinger equation is proposed. The electronic energy is written as a functional of the first-order reduced density matrix, and the diagonal part of the cumulant of the second-order reduced density matrix. A simple approximation for the diagonal part of the cumulant is proposed. In the basis of natural orbitals the energy functional has to be minimized with respect to the molecular orbital coefficients and the natural orbital occupation numbers. A possible energy optimization technique is described. The connection of the proposed density cumulant functional theory (DCFT) with density functional and density matrix functional theories are discussed.
\end{abstract}

\section{I.Introduction}

The main goal of quantum chemistry is to solve the Schrödinger equation for atoms and molecules. By using the Born-Oppenheimer approximation the problem can be reduced to the electronic Schrödinger equation. The last includes the sum of electron kinetic energy, electron-nuclear interaction and electron-electron interaction terms.

The brute force approach to this problem is to use some parameterization for the wave function and determine the values of the parameters using the variational principle or perturbation theory. Even though such an approach can give very accurate energies and wave functions for systems with few electrons, it becomes extremely expensive for larger systems. The tradeoff between the accuracy of such methods and computational expense is possible and widely used for large systems. 
Another widely used approach for large systems is density functional theory (DFT). This theory is based on the theorem, proved by Hohenberg and Kohn, which states that the ground state energy is uniquely determined by the electron density ${ }^{1}$. However, the functional that connects the electron density and the energy is unknown. The currently used version of Kohn-Sham type DFT is based on the adiabatic connection approach in which the kinetic energy is not a (generally unknown) functional of the density, but rather a functional of the first-order reduced density matrix of a system of non-interacting electrons with the same electron density as the system of interest ${ }^{2}$. This results in the fact that the so-called exchangecorrelation functional of DFT includes some part of the kinetic energy of the system.

After considering wave function and DFT approaches one might ask if it is possible to represent the energy as a known functional of some quantity, less complex than the wave function but probably more complex than the electron density. The answer is yes. In fact such a quantity and its exact functional has been known for some time now ${ }^{3}$. The quantity is called the second-order reduced density matrix (2-RDM) and the energy can be expressed as a simple functional of it. This generated considerable excitement and stimulated extensive studies of the 2-RDM in the 1960s and 1970s. However, it was recognized that the 2-RDM has to satisfy an additional condition: it must to be derivable from some properly antisymmetrized n-electron wave function. It was found that this condition is difficult to satisfy without referring to the actual wave function. This complication is called the Nrepresentability problem. Some necessary conditions for the N-representability of the 2-RDM are known, but they are not restrictive enough to permit minimization of the energy with respect to the elements of the 2-RDM.

In 1976 it was shown that the so-called system of coupled k-particles contracted Schrödinger equations ( $k-C S E$ ), which are formulated in terms of the reduced density matrices of order $k, k+1$ and $k+2,(k=1, \ldots, N$, where $N$ is the number of electrons) is equivalent to the Schrödinger equation ${ }^{4,5}$. Therefore, a more promising way to ensure approximate $\mathrm{N}$-representability of the 2-RDM seems to be solving the system of such equations. However, because there is no reason to assume that the n-RDM of any order $\mathrm{n}$ can be neglected, it is necessary to 'reconstruct' the $(k+1)$ - and $(k+2)$-RDMs from the RDMs of order $\mathrm{k}$ and lower ${ }^{6}$. Such a reconstruction procedure was later shown to be equivalent to the 
formulation of the theory in terms of RDMs of order up to $\mathrm{k}$ and cumulants of RDMs of order $\mathrm{k}+1$ and higher ${ }^{7}$. In contrast to RDMs the series of cumulants can be truncated at some order $\mathrm{n}$. Another big advantage of formulating the theory in terms of cumulants is that such a theory is guaranteed to be size-extensive. This property seems to be very important for any theory designed for finite systems and absolutely necessary for treating infinite systems ${ }^{8}$.

Although the solution of the set of k-CSEs written in terms of cumulants seems to be a promising alternative to wave function methods, it has been shown that such an approach is much less effective than perturbation theory or coupled cluster theory based on the wave function approach. This is because in order to get the energy correct to order $\mathrm{n}$ in perturbation theory based on cumulants, it is necessary to use an $n+1$ particle approximation, or $n$ particle approximation if one uses the renormalized hierarchy of $\operatorname{CSE}^{9}$. On the other hand, the wave function based perturbation theory allows one to get the energy correct to $(\mathrm{n}+1)$ order using $\mathrm{n}$ particle approximation ${ }^{10}$.

It is important to point out that there are several different ways, apart from DFT, that the energy can be written as a unknown functional of different quantities. In first-order reduced density matrix functional theory (DMFT) the energy is written as a functional of the 1-RDM only. The exchange energy functional of the 1-RDM is known. The only unknown part of the energy functional is the correlation functional. Because of this the DMFT seems to be more promising then DFT. However, after several simple correlation functionals of the 1RDM were tested it became clear that it is hard to get DFT quality results with DMFT. This may be the result of a fortunate cancellation of exchange and correlation energies errors in DFT. The pair-density functional theory (PDFT) is another recently proposed energy functional $^{11}$. In PDFT the exchange-correlation functional is known, but the kinetic energy functionals are unknown. Another possible way to write an energy functional is to use intracules, functions of the relative positions or momenta of two electrons ${ }^{12}$.

In this work a new method is proposed in which the energy is expressed as a functional of the first order density matrix and the diagonal part (density) of the second order cumulant. This functional can be minimized by imposing simple constrains for $\mathrm{N}$ representability of the 1-RDM. The diagonal part of the second order cumulant is represented by a simple function of the interelectron distance. 
In Section II of this paper the definitions of the RDMs and their cumulants are given. The energy is presented as a functional of these quantities. In Section III a simple approach, based on an approximation of the diagonal part of the cumulant of 2-RDM, is presented. The minimization of the energy functional is discussed in Section IV. In the final section the advantages of the proposed density cumulant functional theory over density functional and density matrix theories are discussed.

\section{Energy as a functional of the 1-RDM and cumulant of 2-RDM}

The first and second order reduced density matrices are defined in coordinate space as

$$
\begin{aligned}
& \gamma_{1}\left(\mathbf{r}_{1}, \mathbf{r}_{1}^{\prime}\right)=N \int \Psi^{*}\left(\mathbf{r}_{1}, \mathbf{r}_{2}, \mathbf{r}_{3}, \ldots, \mathbf{r}_{N}\right) \Psi\left(\mathbf{r}_{1}^{\prime}, \mathbf{r}_{2}, \mathbf{r}_{3}, \ldots, \mathbf{r}_{N}\right) d \mathbf{r}_{2} \ldots d \mathbf{r}_{N} \\
& \gamma_{2}\left(\mathbf{r}_{1}, \mathbf{r}_{2}, \mathbf{r}_{1}^{\prime}, \mathbf{r}_{2}^{\prime}\right)=N(N-1) \int \Psi^{*}\left(\mathbf{r}_{1}, \mathbf{r}_{2}, \mathbf{r}_{3}, \ldots, \mathbf{r}_{N}\right) \Psi\left(\mathbf{r}_{1}^{\prime}, \mathbf{r}_{2}^{\prime}, \mathbf{r}_{3}, \ldots, \mathbf{r}_{N}\right) d \mathbf{r}_{3} \ldots d \mathbf{r}_{N}
\end{aligned}
$$

The energy as a functional of these density matrices can be written as

$$
\begin{aligned}
E= & \int \delta\left(\mathbf{r}-\mathbf{r}^{\prime}\right)\left(-\frac{1}{2} \nabla_{r} \nabla_{r^{\prime}}+V_{e x t}(\mathbf{r})\right) \gamma_{1}\left(\mathbf{r} ; \mathbf{r}^{\prime}\right) d^{3} \mathbf{r} d^{3} \mathbf{r}^{\prime}+ \\
& +\int \gamma_{2}\left(\mathbf{r}_{1}, \mathbf{r}_{2} ; \mathbf{r}_{1}, \mathbf{r}_{2}\right) \frac{1}{r_{12}} d^{3} \mathbf{r}_{1} d^{3} \mathbf{r}_{2}
\end{aligned}
$$

The cumulant of the 2-RDM in coordinate space can be defined as the difference between the 2-RDM and the antisymmetrized product of the 1-RDM.

$$
\lambda_{2}\left(r_{1}, r_{2} ; r_{1}^{\prime}, r_{2}^{\prime}\right)=\gamma_{2}\left(\mathbf{r}_{1}, \mathbf{r}_{2} ; \mathbf{r}_{1}^{\prime}, \mathbf{r}_{2}^{\prime}\right)-\gamma_{1}\left(\mathbf{r}_{1} ; \mathbf{r}_{1}^{\prime}\right) \gamma_{1}\left(\mathbf{r}_{2} ; \mathbf{r}_{2}^{\prime}\right)+\gamma_{1}\left(\mathbf{r}_{1} ; \mathbf{r}_{2}^{\prime}\right) \gamma_{1}\left(\mathbf{r}_{2} ; \mathbf{r}_{1}^{\prime}\right)
$$

Here the antysymmetrized product of the 1-RDM reflects the fact that electrons are fermions and represents exchange interaction between electrons. The cumulant of the 2-RDM contains all information about correlation between electrons. If the cumulant is equal to zero, 
electrons are uncorrelated, and the energy functional (2) is equal to the Hartree-Fock functional.

From (2) it is clear that the energy depends on the 1-RDM and the diagonal part of the 2-RDM only. This diagonal part is usually called a pair-density.

$$
\begin{aligned}
\rho_{2}\left(\mathbf{r}_{1}, \mathbf{r}_{2}\right)=\gamma_{2}\left(\mathbf{r}_{1}, \mathbf{r}_{2} ; \mathbf{r}_{1}, \mathbf{r}_{2}\right) & =\gamma_{1}\left(\mathbf{r}_{1} ; \mathbf{r}_{1}\right) \gamma_{1}\left(\mathbf{r}_{2} ; \mathbf{r}_{2}\right)- \\
& -\gamma_{1}\left(\mathbf{r}_{1} ; \mathbf{r}_{2}\right) \gamma_{1}\left(\mathbf{r}_{2} ; \mathbf{r}_{1}\right)+\lambda_{2}\left(\mathbf{r}_{1}, \mathbf{r}_{2} ; \mathbf{r}_{1}, \mathbf{r}_{2}\right)
\end{aligned}
$$

Using the fact that the diagonal part of the 1-RDM is the electron density and defining $u_{2}\left(\mathbf{r}_{1}, \mathbf{r}_{2}\right)$ as the diagonal part of the cumulant of the 2-RDM, the energy can be expressed as the sum of one- and two-electron energies

$$
\begin{aligned}
& E=E_{1}+E_{2} \\
& E_{1}=\int \delta\left(\mathbf{r}-\mathbf{r}^{\prime}\right)\left(-\frac{1}{2} \nabla_{r} \nabla_{r^{\prime}}+V_{e x t}(\mathbf{r})\right) \gamma_{1}\left(\mathbf{r} ; \mathbf{r}^{\prime}\right) d^{3} \mathbf{r} d^{3} \mathbf{r}^{\prime} \\
& E_{2}=\int \frac{\rho\left(\mathbf{r}_{1}\right) \rho\left(\mathbf{r}_{2}\right)}{r_{12}} d^{3} \mathbf{r}_{1} d^{3} \mathbf{r}_{2}-\int \frac{\gamma_{1}\left(\mathbf{r}_{1} ; \mathbf{r}_{2}\right) \gamma_{1}\left(\mathbf{r}_{2} ; \mathbf{r}_{1}\right)}{r_{12}} d^{3} \mathbf{r}_{1} d^{3} \mathbf{r}_{2}+\int \frac{u_{2}\left(\mathbf{r}_{1}, \mathbf{r}_{2}\right)}{r_{12}} d^{3} \mathbf{r}_{1} d^{3} \mathbf{r}_{2}
\end{aligned}
$$

The two-electron energy is a sum of three terms, which can be associated with Coulomb, exchange and correlation energies respectively. This partition, however, is not entirely equivalent to those that are traditional in either ab-initio theory or DFT, because the expression (5) refers to a non-idempotent 1-RDM (natural orbital occupation numbers can assume any values between 0 and 1). It can be argued that such a partition of the energy functional is more general than the traditional one used in DFT, since it is valid for any type of wave function; the traditional DFT definition assumes that the wave function can be represented as a single Kohn-Sham determinant.

\section{Parametrization of diagonal part of cumulant.}

One possible way to parameterize the diagonal part of the cumulant $u_{2}\left(\mathbf{r}_{1}, \mathbf{r}_{2}\right)$ is 


$$
u_{2}\left(\mathbf{r}_{1}, \mathbf{r}_{2}\right)=\left[\rho\left(\mathbf{r}_{1}\right) \rho\left(\mathbf{r}_{2}\right)-\gamma_{1}\left(\mathbf{r}_{1} ; \mathbf{r}_{2}\right) \gamma_{1}\left(\mathbf{r}_{2} ; \mathbf{r}_{1}\right)\right] G\left(\mathbf{r}_{1}, \mathbf{r}_{2}\right)
$$

where $G\left(\mathbf{r}_{1}, \mathbf{r}_{2}\right)$ represents the correlation part of the pair-correlation function ${ }^{14}$.

This is equivalent to parameterization of the pair-density $\rho_{2}\left(r_{1}, r_{2}\right)$ as

$$
\rho_{2}\left(\mathbf{r}_{1}, \mathbf{r}_{2}\right)=\left[\rho\left(\mathbf{r}_{1}\right) \rho\left(\mathbf{r}_{2}\right)-\gamma_{1}\left(\mathbf{r}_{1} ; \mathbf{r}_{2}\right) \gamma_{1}\left(\mathbf{r}_{2} ; \mathbf{r}_{1}\right)\right]\left[1+G\left(\mathbf{r}_{1}, \mathbf{r}_{2}\right)\right]
$$

In the basis of natural spin-orbitals $\psi_{p}$ (eigenfunctions of 1-RDM) with occupation numbers $n_{p}$ (eigenvalues of 1-RDM), the energy functional can be written as

$$
\begin{aligned}
& E=E_{1}+E_{2} \\
& E_{1}=\sum_{p}\left(-\frac{1}{2} n_{p}\left\langle\psi_{p}\left|\nabla^{2}\right| \psi_{p}\right\rangle+n_{p}\left\langle\psi_{p}\left|V_{e x}\right| \psi_{p}\right\rangle\right) \\
& E_{2}=\sum_{p q}\left(n_{p} n_{q}\left\langle\psi_{p} \psi_{p}\left|r_{12}^{-1}\left(1+G\left(\mathbf{r}_{1}, \mathbf{r}_{2}\right)\right)\right| \psi_{q} \psi_{q}\right\rangle-\frac{1}{2} n_{p} n_{q}\left\langle\psi_{p} \psi_{q}\left|r_{12}^{-1}\left(1+G\left(\mathbf{r}_{1}, \mathbf{r}_{2}\right)\right)\right| \psi_{p} \psi_{q}\right\rangle\right)
\end{aligned}
$$

Here we assume that the natural orbitals are spin-independent, and the natural orbital occupation numbers are equal for $\alpha$ and $\beta$ spin-orbitals.

$$
n_{p}^{\alpha}=n_{p}^{\beta} \equiv n_{p}
$$

The N-representability conditions for the 1-RDM can be written as

$$
\begin{aligned}
& 2 \sum_{p}^{N / 2} n_{p}=N \\
& 0 \leq n_{p} \leq 1
\end{aligned}
$$

Eq (8) has some similarity with density matrix functional theory (DMFT) and density functional theory (DFT), especially non-local $\mathrm{DFT}^{13}$ with Hartree-Fock exchange. However, 
it is clearly different from DMFT because in addition to the 1-RDM, the energy depends on the cumulant function $G\left(\mathbf{r}_{1}, \mathbf{r}_{2}\right)$. The main advantage of this energy partitioning compared with non-local DFT with HF exchange is that the fictional system of non-interacting electrons, related to the real system through an adiabatic connection; is not needed anymore. The non-idempotent character (non-integer NOON) of the 1-RDM basically substitutes for the adiabatic connection in DFT. Because of this, the kinetic energy does not mix with exchange and correlation energies in the energy functional. This means that exchange and correlation are more precisely defined and the function $G\left(\mathbf{r}_{1}, \mathbf{r}_{2}\right)$ has a simple physical meaning. Apart from producing a cleaner theory, it might mean that a simple approximation for $G\left(\mathbf{r}_{1}, \mathbf{r}_{2}\right)$ is possible. It is important to point out that the parameterization (7) guarantees that there is no self-interaction error, which is considered to be one of the major drawbacks of most DFT functionals currently in use.

Here a very simple approximation is proposed for the function $G\left(\mathbf{r}_{1}, \mathbf{r}_{2}\right)$.

$$
G\left(r_{1}, r_{2}\right) \approx G\left(r_{12}\right)=-b_{12} e r f c\left(\omega_{12} r_{12}\right)
$$

Here $\operatorname{erfc}(x)=1-\operatorname{erf}(x)$ is the complementary error function. In general, the parameters $\omega_{12}$ and $b_{12}$ are functions of the electron density. A simple approximation for $\omega_{12}$ proposed by Panas ${ }^{14}$ can be used.

$$
\omega_{12}=\frac{\xi+\kappa}{2}+\sqrt{\left(\frac{\xi+\kappa}{2}\right)^{2}+\xi \kappa}
$$

Here $\xi=\alpha+\beta, \kappa=\chi+\delta$ and $\alpha, \beta, \chi, \delta$ are exponents of gaussian basis functions in a two-electron integral.

The parameters $b_{12}$ are determined from the Kimball cusp condition for the paircorrelation function ${ }^{15}$, 


$$
\left.\frac{\partial G\left(r_{12}\right)}{\partial r_{12}}\right|_{r_{12}=0}=1+G(0)
$$

which yields

$$
b_{12}=\left(1+\frac{2 \omega_{12}}{\sqrt{\pi}}\right)^{-1}
$$

The two-electron energy can be written as

$$
\begin{aligned}
& E_{2}=\sum_{p q}\left(n_{p} n_{q}\left\langle\psi_{p} \psi_{p}\left|\tilde{g}_{12}\right| \psi_{q} \psi_{q}\right\rangle-\frac{1}{2} n_{p} n_{q}\left\langle\psi_{p} \psi_{q}\left|\tilde{g}_{12}\right| \psi_{p} \psi_{q}\right\rangle\right) \\
& \tilde{g}_{12}=\left(1-b_{12}\right) r_{12}^{-1}+b_{12} r_{12}^{-1} e r f\left(\omega_{12} r_{12}\right)
\end{aligned}
$$

The two-electron integrals in equation (15) can be represented as a sum of two types of integrals.

$$
\left\langle\psi_{p} \psi_{p}|\tilde{g}| \psi_{q} \psi_{q}\right\rangle=\left(1-b_{12}\right)\left\langle\psi_{p} \psi_{p}\left|r_{12}^{-1}\right| \psi_{q} \psi_{q}\right\rangle+b_{12}\left\langle\psi_{p} \psi_{p}\left|r_{12}^{-1} \operatorname{erf}\left(\omega_{12} r_{\mathrm{I} 2}\right)\right| \psi_{q} \psi_{q}\right\rangle
$$

The first term on the right side of equation (16) is a normal two-electron integral multiplied by a constant, and it can be calculated with a standard integral code. The second term includes an integral over the error function and can be calculated by modifying a standard integral code ${ }^{14}$. Therefore, this implementation of density cumulant functional theory requires the calculation of twice the number of two-electron integrals needed for HartreeFock or DFT calculations. However, since the two integrals on the right side of equation (16) can be added inside an integral subroutine, the requirement for integral storage in DCFT is the same as that in Hartree-Fock or DFT. 


\section{Optimization of energy functional}

The energy functional (8) must be minimized with respect to the variation of the natural orbitals and their occupation numbers. Because of the fractional occupation numbers of the natural orbitals, this optimization problem cannot be expressed as a simple selfconsistent eigenvalue problem. A direct minimization technique must be used to optimize the values of the occupation numbers and natural orbital coefficients. In addition, the optimization is a subject to several constraints. The natural orbitals have to be kept orthogonal, and the values of the natural orbital occupation numbers (NOON) must be in the range between 0 and 1 , with their sum equal to the number of electrons in the system (Nrepresentability requirment for 1-RDM).

Several different methods of direct optimization have been proposed within the framework of DMFT ${ }^{16,17}$ and self-interaction corrected DFT ${ }^{18}$. For the natural orbital optimization the second order self-consistent field (SOSCF) technique, developed for the orbital improvement step during the optimization of an MCSCF wave function ${ }^{19}$ will be used. Since the functional ( 8 ) is quadratic with respect to natural orbital occupation numbers, the optimization of NOONs can be efficiently accomplished by a second-order Newton-Raphson method. Assuming the natural orbitals are real, the first and second derivatives of the energy with respect to the NOONs are

$$
\begin{aligned}
& \frac{\partial E}{\partial n_{i}}=-\frac{1}{2}\left\langle\psi_{i}\left|\nabla^{2}\right| \psi_{i}\right\rangle+\left\langle\psi_{i}\left|V_{e x t}\right| \psi_{i}\right\rangle+\sum_{q}\left(2 n_{q}\left\langle\psi_{i} \psi_{i}\left|\tilde{g}_{12}\right| \psi_{q} \psi_{q}\right\rangle-n_{q}\left\langle\psi_{i} \psi_{q}\left|\tilde{g}_{12}\right| \psi_{i} \psi_{q}\right\rangle\right) \\
& \frac{\partial^{2} E}{\partial n_{j} \partial n_{i}}=\left\langle\psi_{i} \psi_{i}\left|\tilde{g}_{12}\right| \psi_{j} \psi_{j}\right\rangle-\frac{1}{2}\left\langle\psi_{i} \psi_{j}\left|\tilde{g}_{\mathrm{i} 2}\right| \psi_{i} \psi_{j}\right\rangle
\end{aligned}
$$

Since the values of the NOONs must be kept between 0 and 1 , it is useful to parametrize them as

$$
n_{i}=\left(\cos \theta_{i}\right)^{2}
$$


and optimize $\theta$ without any constraints ${ }^{17}$. The first and second derivatives of the energy with respect to $\theta$ can be calculated using the chain rule.

$$
\begin{aligned}
& \frac{\partial E}{\partial \theta_{i}}=\frac{\partial n_{i}}{\partial \theta_{i}} \frac{\partial E}{\partial n_{i}}=-\sin \left(2 \theta_{i}\right) \frac{\partial E}{\partial n_{i}} \\
& \frac{\partial^{2} E}{\partial \theta_{j} \partial \theta_{i}}=-2 \cos \left(2 \theta_{i}\right) \frac{\partial E}{\partial n_{i}} \delta_{i j}+\sin \left(2 \theta_{i}\right) \sin \left(2 \theta_{j}\right) \frac{\partial^{2} E}{\partial n_{j} \partial n_{i}}
\end{aligned}
$$

It is possible to parametrize the 1-RDM in such a way that the sum of all NOONs is equal to the number of electrons in the system. However, experience with DMFT has shown that it is more practical to satisfy this condition by optimizing the penalized energy function ${ }^{17}$.

$$
\tilde{E}=E+K\left(N-2 \sum_{i}^{N / 2} n_{i}\right)^{2}
$$

Here $\mathrm{K}$ is a large positive constant. After optimizing the energy with respect to the NOONs for a particular value of $\mathrm{K}$, the energy can be reoptimized for a larger value of $\mathrm{K}$ to ensure a good quality 1-RDM. The energy optimization with respect to the NOONs is much faster than optimization with respect to the orbital coefficients, and several iterations with different values of $\mathrm{K}$ will lead to an insignificant increase in computation time.

\section{Preliminary Results}

At present, only DCFT with non-idempotent 1-RDM (all natural orbital occupation numbers are equal to 0 or 1 ) is implemented. As a test for DCFT, the dissociation energies of several diatomic molecules were calculated. The preliminary results of such DCFT calculations with non-idempotent 1-RDM are presented in Table 1. In general DCFT gives better energies than restricted Hartree-Fock (RHF) theory. However, DFT with the B3LYP functional and Mrller-Plesset perturbation theory (MP2) produce significantly more accurate dissociation energies than DCFT. For comparison the results of regularized Hartree-Fock 
(reg-RHF) ${ }^{14}$ calculations are presented in Table 1 as well. Regularized Hartree-Fock is very similar to DCFT with an idempotent matrix, and it was implemented to insure that error function integrals are calculated correctly. However, reg-RHF includes an empirically calculated correction for the kinetic energy functional. In non-idempotent DCFT, such a correction is accounted for by optimizing the values of the natural orbital occupation numbers. The optimization of natural orbital occupation numbers should improve DCFT results significantly.

\section{Summary}

The new method introduced here, called density cumulant functional theory (DCFT), is proposed for solving the stationary electronic Schrödinger equation. In this approach the electronic energy is represented as a functional of the first-order density matrix and the diagonal part of the cumulant of the second-order density matrix. The diagonal part of the cumulant is approximated by a simple function of the interelectron distances. The energy functional is minimized in the basis of natural orbitals with respect to molecular orbital coefficients and natural orbital occupation numbers. Constraints on the values of the natural orbital occupation numbers are used to ensure the N-representability of the first-order density matrix.

Because in DCFT the first-order reduced density matrix is non-idempotent, in contrast with DFT, DCFT need not use the adiabatic connection with introduction of fictitious non-interacting system. DCFT does not suffer from the self-interaction problem. In this respect the DCFT is somewhat similar to density matrix functional theory. The main difference from DMFT is that instead of seeking an approximation of the 2-RDM as a functional of the 1-RDM, the cumulant of the 2-RDM is approximated with a simple function of the interelectron distances.

Since most ab-initio quantum chemical methods rely on the expansion of a wave function in the basis of one-electron functions, they suffer from slow convergence of the energy and other properties as the size of the one-electron basis set increases. This is the result of the inability of such an expansion to describe electron-electron interactions at small 
inter-electronic distances. Since the Kimball cusp condition for the pair-correlation function is explicitly included in the proposed method, its combination with ab-initio methods might prove to be fruitful. In such an approach the short-range electron correlation can, for example, be described by a semi-empirical method, and long-range correlation by an abinitio method. A similar combination of DFT and MRCI has already been shown to be promising ${ }^{20}$.

\section{Acknowledgments}

This work was supported by a grant from the Air Force Office of Scientific Research. The authors thank Dr. Michael Schmidt for fruitful discussions.

\section{References}

${ }^{1}$ P. Hohenberg and W. Kohn, Phys. Rev. 136, B864 (1964).

${ }^{2}$ W. Kohn, L.J. Sham, Phys. Rev. 140, A1133, (1965).

${ }^{3}$ A. J. Coleman, V. I. Yukalov, Reduced Density Matrices, Lecture Notes in Chemistry No

72. (Springer, Berlin, 2000).

${ }^{4}$ H. Nakatsuji, Phys. Rev. A, 14, 41, (1976).

${ }^{5}$ L. Cohen, C. Freshberg. Phys. Rev. A. 13, 927 (1976).

${ }^{6}$ C. Valdemoro, Phys. Rev. A, 45, 971, (1992); C. Valdemoro, L. M. Tel, E. Perez-Romero, Adv. Quant. Chem. 28, 83 (1997).

${ }^{7}$ D. Mazziotti, Phys. Rev. A. 57, 4219, (1998).

${ }^{8} \mathrm{P}$. Fulde, Electron correlations in molecules and solids, (Springer, Berlin, New-York 1995).

${ }^{9}$ W. Kutzelnigg, D. Mukherjee, J. Chem. Phys. 120, 7350, (2004).

${ }^{10}$ I. Levine, Quantum Chemistry, (Prentice-Hall, Inc. Upper Saddle River, NJ, USA, 2000).

${ }^{11}$ P. Ziesche, Int. J. Quantum Chem. 60, 1361 (1996); F. Furche, Phys. Rev. A, 70, 022514 (2004).

${ }^{12}$ P.M.W. Gill, D.P. O'Neill, N.A. Besley, Theor. Chem. Acc. 109, 241 (2003). 
${ }^{13}$ O. Gunnarsson, M. Jonson, B. I. Lundqvist, Phys. Lett. A, 59, 177, (1976); O. Gunnarsson, M. Jonson, B. I. Lundqvist, Solid State Commun. 24, 765, (1977); O. Gunnarsson, M. Jonson, B. I. Lundqvist, Phys. Rev. B, 20, 3136, (1979); J. A. Alonso, L. A. Girifalco. Phys. Rev. B, 17, 3735, (1978).

${ }^{14}$ I. Panas, Chem. Phys. Lett. 245, 171, (1995); I. Panas, A. Snis, Theor. Chem. Acc. 97, 232, (1997).

${ }^{15}$ J. C. Kimball, Phys. Rev. B, 7, 1648, (1973).

${ }^{16}$ A.J. Cohen, E.J. Baerends, Chem. Phys. Lett 364, 409, (2002); J. Cioslowski, K. Pernal, J. Chem. Phys. 115, 5784, (2001); J. Cioslowski, K. Pernal, J. Chem. Phys. 117, 67, (2002).

V.N. Staroverov, G.E. Scuseria, J. Chem. Phys 117, 2489 (2002).

${ }^{17}$ J.M. Herbert, J.E. Harriman, J. Chem. Phys. 118, 10835, (2003).

${ }^{18}$ S Goedecker, C.J. Umrigar, Phys. Rev. A, 55, 1765, (1997).

${ }^{19}$ G. Chaban, M.W. Schmidt, M.S. Gordon, Theor. Chem. Acc. 97, 88, (1997).

${ }^{20}$ T. Leininger, H. Stoll, H-J. Werner, A. Savin, Chem. Phys. Lett. 275, 151, (1997). 
Table 1. Total energies of atoms and diatomic molecules (a.u.) and dissociation energies of diatomic molecules (eV).

\begin{tabular}{lrrrrrr}
\hline \hline & \multicolumn{1}{c}{ RHF } & \multicolumn{1}{c}{ MP2 } & \multicolumn{1}{c}{ B3LYP } & reg-RHF & \multicolumn{1}{c}{ DCFT } & Exp $^{\mathrm{a}}$ \\
\hline $\mathrm{H}$ & -0.499810 & -0.499810 & -0.499810 & -0.499810 & -0.499810 & \\
$\mathrm{H}_{2}$ & -1.132961 & -1.164640 & -1.173238 & -1.147283 & -1.138380 & \\
$\mathrm{D}_{\mathrm{e}}$ & 3.63 & 4.49 & 4.72 & 4.02 & 3.78 & 4.75 \\
& & & & & \\
$\mathrm{~F}$ & -99.400940 & -99.604870 & -99.727140 & -100.216900 & -99.583020 & \\
$\mathrm{~F}_{2}$ & -198.751400 & -199.274400 & -199.517700 & -200.422000 & -199.118300 & \\
$\mathrm{D}_{\mathrm{e}}$ & -1.37 & 1.76 & 1.73 & -0.32 & -1.30 & 1.66 \\
& & & & & & \\
$\mathrm{~N}$ & -54.259610 & -54.374550 & -54.472720 & -54.610040 & -54.347120 & \\
$\mathrm{~N}_{2}$ & -108.983400 & -109.357200 & -109.516100 & -110.973600 & -109.340200 & \\
$\mathrm{D}_{\mathrm{e}}$ & 12.63 & 16.55 & 15.53 & 47.72 & 17.58 & 9.91 \\
\hline \hline
\end{tabular}

"Experimental values are taken from Ref. [14] 


\section{CHAPTER 7. GENERAL CONCLUSIONS}

This work describes the applications of ab-initio quantum chemical methods to the studies of atomic clusters. In Chapter 1 a general description of quantum chemical methods, used to solve the stationary Schrödinger equation in subsequent parts of the dissertation, is given. The Born-Oppenheimer approximation allows one to separate the problem into two parts: nuclear and electronic. The Hartree-Fock method, based on the mean field approximation, is the simplest approach to solution of the electronic Schrödinger equation. Electron correlation is defined as the difference between the exact and Hartree-Fock solutions of the electronic Schrödinger equation. Several methods to recover correlation energy using post-Hartree-Fock methods such as configuration interaction, perturbation theory and the coupled cluster approach are discussed. When strong electron correlations are present the Hartree-Fock wave function becomes a poor zeroth-order approximation. The best way to treat such systems is to use the multiconfigurational self-consistent field (MCSCF) wave function to describe strongly correlated electrons. The remaining electron correlation can be recovered with multireference versions of configuration interaction or perturbation theory. Density functional theory is an alternative approach to solve the manyelectron problem. Unfortunately, the present implementations of DFT are unable to describe strong electron correlation. In addition, the introduction of parameters into DFT functionals makes it very hard to judge the quality of results that are obtained using this method.

In Chapter 2 the adsorption of oxygen molecules on small neutral and anionic gold clusters is studied. It is shown that $\mathrm{O}_{2}$ binds better to clusters with an odd number of electrons than to clusters with an even number of electrons. DFT results are found to be in significant disagreement with high-level ab-initio $\operatorname{CCSD}(\mathrm{T})$ results. In contrast the $\operatorname{CCSD}(\mathrm{T})$ results are in good agreement with experimental measurements. The DFT binding energy errors seem to be erratic and difficult to correct by any empirical scheme.

Chapter 3 describes the study of reaction mechanisms of molecular hydrogen with small neutral and anionic gold clusters. The binding energies of one and two $\mathrm{H}_{2}$ molecules are calculated. The transition states of $\mathrm{H}_{2}$ dissociation on gold clusters are located. In contrast 
to $\mathrm{O}_{2}$ absorption, DFT produces reasonable results for the $\mathrm{H}_{2}$ binding energies and the barriers to $\mathrm{H}_{2}$ dissociation.

The study of the stability of different isomers of $\mathrm{C}_{36}$ carbon clusters is presented in Chapter 4. It is shown that the singlet state of the lowest energy $D_{6 h}$ isomer has significant diradical character. This results in the failure of Hartree-Fock and DFT methods to predict the correct lowest energy isomer and its electronic ground state. The experimental data is explained based on multireference perturbation theory calculations. It is shown that strong electron correlation is responsible for the high stability of $D_{6 h}$ isomer observed in experiments. The artificial distortion of the $\mathrm{D}_{6 \mathrm{~h}}$ isomer to lower symmetry structures at the Hartree-Fock level of theory is explained by an oversimplified form of the wave function.

In Chapter 5 the mixed metal-carbon $\mathrm{Ti}_{8} \mathrm{C}_{12}$ cluster is studied with the main goal to determine the geometry and ground electronic state of this cluster. It is shown that the $T_{d}$ structure with a ${ }^{1} \mathrm{E}$ ground state is a subject to Jahn-Teller distortion. The distorted $\mathrm{D}_{2 \mathrm{~d}}$ and $\mathrm{C}_{3}$, structures are studied with multireference configuration interaction and coupled cluster methods. The $D_{2 d}$ structure with a singlet ground state suggested as a ground state of the $\mathrm{Ti}_{8} \mathrm{C}_{12}$ cluster. Several low-lying singlet and triplet states were found to be nearly degenerate with the ground state. The wave functions of several states have very significant multireference character. Therefore it is surprising that density functional theory is able to provide qualitatively correct results for relative energies of the electronic states. However, DFT predicts distortions of $\mathrm{Ti}_{8} \mathrm{C}_{12}$ to the low symmetry structures, up to $\mathrm{C}_{1^{*}}$ This indicates that DFT is not flexible enough to predict correct geometry of $\mathrm{Ti}_{8} \mathrm{C}_{12}$ cluster.

A new approach for solving the many electron Schrödinger equation is proposed in Chapter 6. In contrast to the wave function or the density functional theory approaches, the proposed method uses the first-order reduced density matrix and the diagonal part (density) of the cumulant of the second-order reduced density matrix. The advantages of such a functional over density functional theory and other approaches are discussed.

In this work the successful use of high-level ab-initio quantum chemical methods for the studies of electronic and atomic structures of metal, carbon and mixed metal-carbon clusters were demonstrated. It was shown that it is often necessary to use such high level of theory to explain the existing experimental data and to predict the properties of new clusters. 
On the other hand, the simple methods such as density functional theory sometimes can produce good qualitative and even quantitatively correct results for atomic clusters. However, since strong electron correlation often plays an important role in such systems, the failure of density functional theory to produce reliable results is common. Therefore, the density functional theory, which are currently very popular in chemistry and especially solid state physics, has to be applied with caution in atomic cluster studies, and its results have to be confirmed with ab-initio methods every time than it is possible. 


\section{ACKNOWLEDGMENTS}

This work would not be possible without the support and guidance of my major professor Dr. Mark S. Gordon. I would like to acknowledge the former and present students of Gordon's group for useful discussions at the Iowa State University and at different meetings and conferences. The discussions with Michael Schmidt, Dmitri Fedorov, Mike Pak and Ryan Olson had significant influence on this work. And finally, I would like to thank my parents Aleksandr and Nina and my wife Elena for patience and support. 